\title{
UBIQUITY OF KOSTKA POLYNOMIALS
}

\author{
ANATOL N. KIRILLOV \\ Graduate School of Mathematics, Nagoya University \\ Chikusa-ku, Nagoya 486-8602, Japan \\ and \\ Steklov Mathematical Institute, \\ Fontanka 27, St.Petersburg, 191011, Russia
}

\begin{abstract}
We report about results revolving around Kostka-Foulkes and parabolic Kostka polynomials and their connections with Representation Theory and Combinatorics. It appears that the set of all parabolic Kostka polynomials forms a semigroup, which we call Liskova semigroup. We show that polynomials frequently appearing in Representation Theory and Combinatorics belong to the Liskova semigroup. Among such polynomials we study rectangular $q$-Catalan numbers; generalized exponents polynomials; principal specializations of the internal product of Schur functions; generalized $q$-Gaussian polynomials; parabolic Kostant partition function and its $q$-analog; certain generating functions on the set of transportation matrices. In each case we apply rigged configurations technique to obtain some interesting and new information about Kostka-Foulkes and parabolic Kostka polynomials, Kostant partition function, MacMahon, Gelfand-Tsetlin and Chan-Robbins polytopes. We describe certain connections between generalized saturation and Fulton's conjectures and parabolic Kostka polynomials; domino tableaux and rigged configurations. We study also some properties of $l$-restricted generalized exponents and the stable behaviour of certain Kostka-Foulkes polynomials.
\end{abstract}

\section{CONTENTS}

1 Introduction

2 Kostka-Foulkes polynomials and weight multiplicities

2.1 Kostka-Foulkes polynomials

2.2 Skew Kostka-Foulkes polynomials

2.3 Weight multiplicities

3 Parabolic Kostka polynomials

4 Fermionic formula for parabolic Kostka polynomials

5 Generalized exponents and mixed tensor representations

6 Internal product of Schur functions

7 Liskova semigroup

7.1 Realizable polynomials and product formula

7.2 Generalized $q$-Gaussian coefficients

7.3 Multinomial coefficients

7.4 Kostka-Foulkes polynomials and transportation matrices

7.5 Gelfand-Tsetlin polytope and volume of weight subspaces

8 Stable behavior of Kostka-Foulkes polynomials 


\section{Introduction}

The Kostka-Foulkes polynomials $K_{\lambda \mu}(q)$ are defined as the matrix elements of the transition matrix which expresses the Schur functions $s_{\lambda}(x)$ in terms of the Hall-Littlewood symmetric functions $P_{\mu}(x ; q)$ :

$$
s_{\lambda}(x)=\sum_{\mu} K_{\lambda \mu}(q) P_{\mu}(x ; q)
$$

To our knowledge, at the first time the polynomials $K_{\lambda \mu}(q)$ had appeared implicitly in the middle of fifties in the familiar series of papers by Green [18, 19] about computation of the characters of the general linear groups over finite fields and their connections with the Hall-Steinitz algebra. The famous Green polynomials $Q_{\rho}^{\lambda}(q)$ are related to the polynomials $K_{\lambda \mu}(q)$ via the following relation

$$
q^{n(\mu)} Q_{\rho}^{\mu}\left(q^{-1}\right)=\sum_{\lambda} \chi_{\rho}^{\lambda} K_{\lambda \mu}(q)
$$

where $\chi_{\rho}^{\lambda}$ is the value of the irreducible character $\chi^{\lambda}$ of the symmetric group $S_{n}$ at elements of cycle-type $\rho$. Later in the paper [47] D.E. Littlewood introduced the symmetric functions

$$
Q_{\lambda}^{\prime}(x ; q)=\prod_{1 \leq i<j \leq n}\left(1-q R_{i j}\right)^{-1} s_{\lambda}(x),
$$

where $R_{i j} s_{\lambda}(x):=s_{R_{i j}(\lambda)}(x)$, and $R_{i j}(\lambda)=\left(\lambda_{1}, \ldots, \lambda_{i}+1, \ldots, \lambda_{j}-1, \ldots, \lambda_{n}\right)$ is the socalled raising operator, see e.g. [52], Chapter I, $\S 1$. Here and further $s_{\lambda}(x)$ denotes the Schur function corresponding to a partition (or composition) $\lambda$, and to the set of variables $x_{1}, \ldots, x_{n}$. In the same paper D.E. Littlewood also computed a few examples of the decomposition of function $Q_{\lambda}^{\prime}(x ; q)$ in terms of Schur functions. The functions $Q_{\lambda}^{\prime}(x ; q)$ are called modified Hall-Littlewood polynomials and related with polynomials $K_{\lambda \mu}(q)$ via a simple relation

$$
Q_{\mu}^{\prime}(x ; q)=\sum_{\lambda} K_{\lambda \mu}(q) s_{\lambda}(x)
$$

Polynomials $K_{\lambda \mu}(q)$ also appeared implicitly, via the generalized exponents of certain modules, in a fundamental paper by Kostant [12].

Probably, A.O. Morris [53] was the first who introduced a special notation $f_{\lambda \mu}(t)$ for the transition coefficients between the modified Hall-Littlewood polynomials $Q_{\lambda}^{\prime}(x ; t)$ and Schur functions $s_{\mu}(x)$, i.e. for Kostka-Foulkes polynomials. Using the Littlewood formula (1.2), A.O. Morris discovered an important recurrence relation between the polynomials $K_{\lambda \mu}(q)$, and computed all those polynomials with $|\lambda|=|\mu| \leq 4$.

In a survey paper 12 H.O. Foulkes at the first time, as far as I am aware, gave rise the problem of non-negativity of the coefficients of polynomials $K_{\lambda \mu}(q)$. He stated a problem "to associate each Young tableau with a precise index of $q$ ". This problem was settled by A. Lascoux and M.-P. Schützenberger [44, 63] who suggested the name "Foulkes polynomials" for polynomials $K_{\lambda \mu}(q)$. Later they renamed these polynomials by Foulkes-Green polynomials, see e.g. 45, and C.R. Acad. Sci., Paris 288 (1979), 95-98. 
In the middle of seventies the polynomials $K_{\lambda \mu}(q)$ had appeared in Algebraic Geometry and Representation Theory in a connection with the study of the so-called "variety of the $N$ stable complete flags $\mathcal{B}_{N}$ "; the crucial papers here are those by R. Steinberg (1976), R. Hotta and T. Springer (1977), T. Springer (Inv. Math. 36 (1976), 173-207), H. Kraft (1981), and C. De Concini and C. Procesi (1981). Precise references may be found in [15]. In a paper by Garsia and Procesi [15 the polynomials $K_{\lambda \mu}(q)$ are named by $q$-Kostka polynomials.

There exists a vast body of literature about the Schur and Hall-Littlewood functions, their numerous applications to Representation theory of the symmetric and general linear groups [52, 59, 54], to Enumerative combinatorics [66], Algebraic geometry [13, 15], to Integrable systems [27, 39, 55], .... But it is surprisingly enough that till now there are no books or survey articles (except [10]) which would describe a big variety of applications of KostkaFoulkes polynomials to different areas of Mathematics and Mathematical Physics.

The present paper is an attempt to collect together some results about the Kostka-Foulkes and parabolic Kostka polynomials related mainly to Representation theory and Combinatorics. Thus, we almost omitted very interesting connections of polynomials $K_{\lambda \mu}(q)$ with Algebraic geometry, as well as with Integrable systems. Some examples of such connections one may find in 15, 26, 33, 34, 43, 40, 55, 61, 62, 69.

The main problems we are going to consider and study in the present paper are Identification and Characterization problems.

i) Characterization problem: how to characterize polynomials with non-negative integer coefficients which might appear to be a Kostka-Foulkes (or parabolic Kostka) polynomials $K_{\lambda \mu}(q)$ (or $\left.K_{\lambda R}(q)\right)$ for some partitions $\lambda$ and $\mu$ (or for some partition $\lambda$ and dominant sequence of rectangular shape partitions $R)$ ?

If such an occasion happened, i.e.

$$
P(q) \stackrel{\bullet}{=} K_{\lambda R}(q)
$$

one can apply fermionic formula for polynomials $K_{\lambda \mu}(q)$ (or $K_{\lambda R}(q)$ ), see Theorem 4.3 and Corollary 4.4, to obtain a deep information about polynomial $P(q)$. Typical example is the generalized binomial coefficients $\left[\begin{array}{c}N \\ \lambda\end{array}\right]_{q}$. One can prove 32 that

$$
\left[\begin{array}{l}
N \\
\lambda^{\prime}
\end{array}\right]_{q} \doteq K_{\widetilde{\lambda}, \widetilde{\mu}}(q)
$$

where $\widetilde{\lambda}=(N|\lambda|, \lambda), \widetilde{\mu}=\left(|\lambda|^{N+1}\right)$, and the symbol $\doteq$ is explained in the list of special notation (Notatition) at the end of Introduction. In this way one comes to the following identity

$$
\left[\begin{array}{l}
N \\
\lambda^{\prime}
\end{array}\right]_{q}=\sum_{\{\nu\}} q^{c(\nu)} \prod_{j, k \geq 1}\left[\begin{array}{c}
P_{j}^{(k)}(\nu)+m_{j}\left(\nu^{(k)}\right) \\
m_{j}\left(\nu^{(k)}\right)
\end{array}\right]_{q},
$$

summed over all sequences of partitions $\{\nu\}=\left\{\nu^{(1)}, \nu^{(2)}, \ldots\right\}$ such that

$$
\text { • }\left|\nu^{(k)}\right|=\sum_{j \geq k} \lambda_{j}, k \geq 1, \nu^{(0)}:=\emptyset
$$


- $P_{j}^{(k)}(\nu):=j(N+1) \delta_{k, 1}+Q_{j}\left(\nu^{(k-1)}\right)-2 Q_{j}\left(\nu^{(k)}\right)+Q_{j}\left(\nu^{(k+1)}\right) \geq 0$, for all $k, j \geq 1$;

- $m_{j}\left(\nu^{(k)}\right)$ is the number of parts of partition $\nu^{(k)}$ of size $j$;

- $c(\nu)=n\left(\nu^{(1)}\right)-n(\lambda)+\sum_{k, j \geq 1}\left(\begin{array}{c}\left(\nu^{(k)}\right)_{j}^{\prime}-\left(\nu^{(k+1)}\right)_{j}^{\prime} \\ 2\end{array}\right)$;

- $\left[\begin{array}{c}n \\ m\end{array}\right]_{q}=\frac{(q ; q)_{n}}{(q ; q)_{m}(q ; q)_{n-m}}$, if $0 \leq m \leq n$, and equals to 0 otherwise.

The identity (1.4) is a highly non-trivial identity, which generalizes the so-called $\mathrm{KOH}$ identity, see e.g. [71, 32, 35], or Section 4, (4.5). The only known proof of (1.4) is a combinatorial one and based on a construction of the rigged configurations bijection, see [32, 35]. For further generalization of the identity (1.4) see Section 6, (6.13), or [35], Section 10.1. It was shown [ibid] that "a constructive proof of the unimodality of the Gaussian coefficients", which is due to O'Hara [56], admits a natural interpretation in terms of rigged configurations. Note also, that identity (1.4) leads to "a one-line high school algebra proof" of the unimodality of the generalized Gaussian polynomials $\left[\begin{array}{l}n \\ \lambda\end{array}\right]_{q}$ (cf. D. Zeilberger, IMA volumes in Math. and Appl., 18 (1989), 67-75).

Characterization problem appears to be a rather difficult one, and we don't know a complete list of conditions for a given polynomial $P(q) \in \mathbb{N}[q]$ to be a parabolic Kostka polynomial. Examples show that in general the sequence of coefficients of a parabolic Kostka polynomial $K_{\lambda R}(q)$ may be not symmetric or unimodal, and may have gaps, i.e. if

$$
K_{\lambda R}(q)=q^{\bullet}\left(a_{0}+a_{1} q+\cdots+a_{m} q^{m}\right), \quad a_{0} a_{m} \neq 0,
$$

then it may be exist an index $j, 1 \leq j \leq m-1$, such that $a_{j}=0$. Nevertheless, it seems plausible the following

Conjecture 1.1 Let

$$
P(q)=\sum_{k=n}^{m} a_{k} q^{k}
$$

be a polynomial with non-negative integer coefficients such that $a_{n} a_{m} \neq 0, a_{n-1}=0$. If there exist non-negative integers $i$ and $j, n \leq i<j<m$, such that $j-i \geq 2$, and $a_{i-1} \leq a_{i}>$ $a_{i+1} \geq \cdots \geq a_{j}<a_{j+1}$, then the polynomial $P(q)$ can't be equal to any parabolic Kostka polynomial.

ii) Identification problem: even though an answer to the Characterization problem is still unknown, it is possible to identify some polynomials, which frequently appear in Combinatorics and Representation theory, with certain parabolic Kostka polynomials.

One of the main goals of our paper is to show that among polynomials, which occur as parabolic Kostka polynomials, are the following:

- rectangular q-Catalan numbers, Section 20, Exercise 1;

- generalized exponents polynomials, Theorem 5.3;

- principal specialization of the internal product of Schur functions, Theorem 6.6; 
- generalized $q$-binomial coefficients, Section 7.2;

- $q$-multinomial and $q^{2}$-multinomial coefficients, Section 7.3;

- q-analog of Kostant's partition function, Section 7, Exercise 2;

- certain generating functions on the set of transportation matrices, Section 7.4;

- polynomials $n(1+q)^{m}$, where $n \geq 1$ and $m \geq 2$ are integer numbers, Section 1 , Exercise 6.

The main purpose of the present paper is to study combinatorial properties of these polynomials, which follow from identification of the polynomials above with certain parabolic Kostka polynomials, by means of the rigged configurations bijection.

Let us say a few words about the content of our paper.

In Section 2 we recall definitions of Kostka-Foulkes polynomials, weight multiplicities, and $q$-weight multiplicities. Exercises to Section 2 include, among others, definitions and basic properties of the rectangular $q$-Catalan and $q$-Narayana numbers, computation of the volume and $\delta$-vector of MacMahon's polytope.

In Section 3 we recall the definition of parabolic Kostka polynomials which are natural generalization of the Kostka-Foulkes polynomials. More details and proofs may be found in [40. Exercises to Section 3 include, among others, a $q$-analog of a result by J. Stembridge 67] about the number of up-down staircase tableaux, see Exercise 7; connection between Littlewood-Richardson's numbers $c_{\lambda \mu}^{\nu}$ and certain parabolic Kostka polynomials, see Exercise 3; various combinatorial properties of the convex polytope

$$
\mathcal{P}_{n}=\left\{\left(x_{1}, \ldots, x_{n}\right) \in \mathbb{R}_{+}^{n} \mid x_{i}+x_{i+1} \leq 1,1 \leq i \leq n-1\right\},
$$

including fermionic formulae for the number $i\left(\mathcal{P}_{n} ; k\right)$ of integer points inside of the polytope $k \mathcal{P}_{n}$; connections with restricted Kostka polynomials and alternating permutations, see Exercise 4.

In Section 4 we recall a fermionic formula for parabolic Kostka polynomials. Proof of the fermionic formula for parabolic Kostka polynomials is based on the study of combinatorial properties of the rigged configurations bijection, and may be found in [41], see also [30. Exercises to Section 1 include, among others, an explanation of certain connections between domino and $p$-ribbon tableaux on one hand and parabolic Kostka polynomials and rigged configurations on the other, see Exercises 3 and 4; "exotic examples" of parabolic Kostka polynomials, which have a few non-zero terms only. It is a very interesting and difficult problem to find all parabolic Kostka polynomials with 2,3 or 4 non-zero terms.

In Section 5 we study the generalized exponents polynomials for some representations of the Lie algebra $\operatorname{sl}(n)$. The generalized exponents polynomials were introduced and studied in the familiar paper by Kostant [42] in a connection with the investigation of the structure of the symmetric algebra of the adjoint representation. Our main observation is that the generalized exponents polynomial $G_{N}\left(V_{\alpha} \otimes V_{\beta}^{*}\right)$ corresponding to the tensor product $V_{\alpha} \otimes V_{\beta}^{*}$ of irreducible $\mathfrak{g l}(N)$-representation $V_{\alpha}$ and the dual $\mathfrak{g} l(N)$-representation $V_{\beta}^{*}$, after multiplication by some power of $q$, coincides with a certain parabolic Kostka polynomial, see Theorem 5.3. This observation leads to a fermionic formula for generalized exponents polynomial corresponding to $\mathfrak{g} l(N)$-representations of the form $V_{\alpha} \otimes V_{\beta}^{*}$ and gives the first real means for computing polynomials $G_{N}\left(V_{\alpha} \otimes V_{\beta}^{*}\right)$. We give also a generalization of the Gupta-Hessenlink-Peterson 
theorem (Theorem 5.1) to the case of so-called $l$-restricted generalized exponents polynomial, see Definition 5.9 and Theorem 5.12 .

In Section 6 we study the internal product of Schur functions and its principal specializations. It looks a challenging problem to find and prove an analog of the LascouxSchützenberger theorem about Kostka-Foulkes polynomials (see e.g. [52], Chapter III, (6.5), (i)) for the transition coefficients $L_{\alpha \beta}^{(\mu)}(q)$ between the internal product of Schur functions and Hall-Littlewood polynomials

$$
s_{\alpha} * s_{\beta}(x)=\sum_{\mu} L_{\alpha \beta}^{(\mu)}(q) P_{\mu}(x ; q)
$$

see Section 6, Problem 6.5, for further details.

Polynomials $L_{\alpha \beta}^{(\mu)}(q)$ have many interesting properties (see Proposition 6.3 and Exercises $\mathbf{1}$ and 2 to Section 6), and may be considered as a natural generalization of the Kostka-Foulkes polynomials $K_{\alpha \mu}(q)$. Note that numbers

$$
L_{\alpha \beta}^{(\mu)}(0)=g_{\alpha \beta \mu}
$$

are equal to the structural constants for multiplication of the characters of the symmetric $\operatorname{group} S_{|\alpha|}$ :

$$
\chi^{\alpha} \chi^{\beta}=\sum_{\mu} g_{\alpha \beta \mu} \chi^{\mu} .
$$

Recall that it is an important open problem to obtain a nice combinatorial interpretation of the numbers $g_{\alpha \beta \mu}$. Based on an observation, see our Theorem 6.6, that the principal specialization of a Schur function after multiplication by some power of $q$, coincides with a certain parabolic Kostka polynomial, we obtain a new proof, see Corollary 6.7, of a fermionic/combinatorial formula for the principal specialization of Schur functions first obtained in [31.

In Section 7 we study Identification problem. Our first result states that the set of all polynomials, which may occur as a parabolic Kostka polynomial, forms a semigroup, i.e. closed under multiplication. We call this semigroup by Liskova semigroup. We show that Liskova semigroup contains the generalized $q$-Gaussian polynomials $\left[\begin{array}{c}n \\ \lambda^{\prime}\end{array}\right]_{q}, q-$ multinomial coefficients $\left[\begin{array}{c}N \\ n_{1}, \ldots, n_{k}\end{array}\right]_{q}, q$-analog of Kostant's partition function, and polynomials related to the Robinson-Schensted-Knuth correspondence between the set of transportation matrices and that of pairs of semistandard Young tableaux of the same shape. More precisely, let $\lambda$ and $\mu$ be compositions whose lengths do not exceed $n$. Denote by $\mathcal{P}_{\lambda \mu}$ the set of all $n$ by $n$ matrices of non-negative integers with row sums $\lambda_{i}$ and column sums $\mu_{j}$ (the so-called set of transportation matrices of type $(\lambda ; \mu))$. The Robinson-Schensted-Knuth correspondence establishes a bijection

$$
\mathcal{P}_{\lambda \mu} \simeq \coprod_{\eta} S T Y(\eta, \mu) \times S T Y(\eta, \lambda)
$$


between the set of transportation matrices $\mathcal{P}_{\lambda \mu}$ and that of all pairs $(P, Q)$ of semistandard Young tableaux of the same shape and weights $\mu$ and $\lambda$ correspondingly.

Thus, for any transportation matrix $m \in \mathcal{P}_{\lambda \mu}$ one can associate the following statistics:

- left charge $c_{L}(m)=c(P)$,

- right charge $c_{R}(m)=c(Q)$,

- total charge $c(m)=c(P)+c(Q)$.

It is well-known that the generating function

$$
\sum_{m \in \mathcal{P}_{\lambda \mu}} q^{c_{R}(m)}=\sum_{\eta} K_{\eta \mu} K_{\eta \lambda}(q):=\mathcal{P}_{\lambda \mu}(q)
$$

describes

i) dimensions of cohomology groups of the partial unipotent flag variety $\mathcal{F}_{\mu}^{\lambda}$

$$
q^{n(\lambda)} \mathcal{P}_{\lambda \mu}\left(q^{-1}\right)=\sum_{k \geq 0} \operatorname{dim} H^{2 k}\left(\mathcal{F}_{\mu}^{\lambda} ; \mathbb{Q}\right) q^{k},
$$

see e.g., Hotta R. and Shimomura N., Math. Ann. 241 (1979), 193-208, or [43, 34];

ii) unrestricted one dimensional sum corresponding to the highest weight $\lambda$ and the tensor product of one row representations

$$
V_{\left(\mu_{1}\right)} \otimes V_{\left(\mu_{2}\right)} \otimes \cdots \otimes V_{\left(\mu_{l}\right)}
$$

where $\mu=\left(\mu_{1}, \ldots, \mu_{l}\right)$ and $l=l(\mu)$. For definition and basic properties of one dimensional sums, their connections with Kostka-Foulkes polynomials, with crystal basis, and integrable models, see e.g. 26 and the literature quoted therein;

iii) the number $\alpha_{\lambda}(S ; p)$ of chains of subgroups

$$
\{e\} \subseteq H^{(1)} \subseteq \cdots \subseteq H^{(m)} \subseteq G
$$

in a finite abelian $p$-group of type $\lambda$ such that each subgroup $H^{(i)}$ has order $p^{a_{i}}, 1 \leq i \leq m$ :

$$
p^{n(\lambda)} \alpha_{\lambda}\left(S ; p^{-1}\right)=\mathcal{P}_{\lambda \mu}(p),
$$

where $S=\left\{a_{1}<a_{2}<\cdots<a_{m}\right\}$ is a subset of the interval $[1,|\lambda|-1]$, and

$$
\mu:=\mu(S)=\left(a_{1}, a_{2}-a_{1}, \ldots, a_{m}-a_{m-1},|\lambda|-a_{m}\right),
$$

see e.g. [5], or [34;

iv) the number of rational points $\mathcal{F}_{\mu}^{\lambda}\left(\mathbb{F}_{q}\right)$ over the finite field $\mathbb{F}_{q}$ of the partial unipotent flag variety $\mathcal{F}_{\mu}^{\lambda}$ :

$$
\mathcal{F}_{\mu}^{\lambda}\left(\mathbb{F}_{q}\right)=q^{n(\lambda)} \mathcal{P}_{\lambda \mu}\left(q^{-1}\right)
$$

In particular, if $\lambda=\left(1^{n}\right)$ then

$$
\mathcal{P}_{\left(1^{n}\right) \mu}(q)=q^{n\left(\mu^{\prime}\right)}\left[\begin{array}{c}
n \\
\mu_{1}, \ldots, \mu_{n}
\end{array}\right],
$$

see e.g. 2.25), and hence $\mathcal{P}_{\left(1^{n}\right) \mu}(q)$ belongs to the Liskova semigroup.

Question. Is it true that polynomials $\mathcal{P}_{\lambda \mu}(q)$ belong to the Liskova semigroup for all partitions $\lambda$ and compositions $\mu$ ? 
Conjecture 1.2 Let $P(q)$ be a polynomial with non-negative integer coefficients and nonzero constant term. There exist two polynomials $f_{1}(q)$ and $f_{2}(q)$ both belonging to the Liskova semifroup, such that $P(q)=f_{1}(q) / f_{2}(q)$.

For a "strong version" of this conjecture, see Section 7.1.

In Section 7.4 we study the generating functions

$$
\begin{aligned}
\sum_{m \in \mathcal{P}_{\lambda \mu}} q^{c(m)} & =\sum_{\eta} K_{\eta \mu}(q) K_{\eta \lambda}(q), \\
\sum_{m \in \mathcal{R}_{\lambda \mu}} q^{c(m)} & =\sum_{\eta} K_{\eta^{\prime} \mu}(q) K_{\eta \lambda}(q),
\end{aligned}
$$

where $\boldsymbol{\mathcal { R }}_{\lambda \mu}$ denotes the set of all $(0,1)$-matrices of size $n$ by $n$ with row sums $\lambda_{i}$ and column sums $\mu_{j}$.

It happens that the generating function (1.7) coincides with a certain Kostka-Foulkes polynomial, see Theorem 7.13, whereas the generating function (1.8) coincides with a certain "super" Kostka-Foulkes polynomial, see Theorem 7.14.

In Section 7.5 for any two partitions $\lambda$ and $\mu$ of the same size, we introduce Ehrhart polynomial $\mathcal{E}_{\lambda \mu}(t)$ of the weight subspace $V_{\lambda}(\mu)$. Namely, using the specialization $q=1$ of fermionic formula (4.2) for Kostka's polynomials, it is not difficult to see that the Kostka number $K_{l \lambda, l \mu}$ is a polynomial in $l$ with integer coefficients. Based on examples, we state a conjecture that Ehrhart's polynomial $\mathcal{E}_{\lambda \mu}(t)$ has in fact non-negative integer coefficients. This conjecture would follow from the general theory of Ehrhart's polynomials, see e.g. [25, 66], if it would be known that the Gelfand-Tsetlin polytope $G(\lambda, \mu)$, see Section 7.5 , is an integral one (this is the so-called Berenstein-Kirillov conjecture, see [38], p.101, Conjecture 2.1). More generally, it follows from the $q=1$ case of the fermionic formula (4.1) that for any partition $\lambda$ and any sequence of rectangular shape partitions $R$ the parabolic Kostka number $K_{l \lambda, l R}$ is a polynomial $\mathcal{E}_{\lambda, R}(l)$ in $l$ with integer coefficients. Based on examples, we make a conjecture that the polynomial $\mathcal{E}_{\lambda, R}(t)$ has in fact non-negative integer coefficients. We expect that the similar statements are valid for (generalized) parabolic Kostka numbers $K_{l \lambda, l \mu, \eta}:=\left.K_{l \lambda, l \mu, \eta}(q)\right|_{q=1}$.

It looks a challenging problem to find an explicit formula for the Ehrhart polynomials $\mathcal{E}_{\lambda \mu}(t)$. This problem should be a very difficult one, however, since, for example, the polynomial $\mathcal{E}_{\left(n^{n}\right),\left((n-1)^{n}, 1^{n}\right)}(t)$ coincides with the Ehrhart polynomial of the Birkhoff polytope $\mathcal{B}_{n}$, see Example 7.19. The (normalized) leading coefficient of polynomial $\mathcal{E}_{\lambda \mu}(t)$ is equal to the (normalized) volume of Gelfand-Tsetlin's polytope $G(\lambda, \mu)$, and is known in the literature (see e.g., G.J. Heckman, Inv. Math. 67 (1982), 333-356) as a continuous analog of the weight multiplicity $\operatorname{dim} V_{\lambda}(\mu)$.

Finally, in the Exercises to Section 7 among others, we study some properties of the parabolic Kostant partition function $K_{\Phi(\eta)}(\gamma ; 1)$, its $q$-analog, as well as its connections with parabolic Kostka polynomials, and the volume of so-called Chan-Robbins polytope, see Exercise 2. Kostant's partition function was introduced and studied by F.A. Berezin and I.M. Gelfand (Proc. Moscow Math. Soc. 5 (1956), 311-351) for the case $\mathfrak{g}=\operatorname{sl}(n)$, and by 
B. Kostant (Trans. Amer. Math. Soc., 93 (1959), 53-73) for arbitrary semi-simple finite dimensional Lie algebra $\mathfrak{g}$. In [36] we had considered in details algebraic and combinatorial properties of the Kostant partition function $K_{\left(1^{n}\right)}(\gamma ; 1)$, and, in particular, computed the values of function $K_{\left(1^{n}\right)}(\gamma ; 1)$ for certain vectors $\gamma$, see Exercise $2 \mathbf{c}$, e- $\mathbf{g}$ to Section 7 . It is not difficult to see (Exercise $\mathbf{2 d}$ to Section 7) that if

$$
\gamma=\left(\gamma_{1}, \ldots, \gamma_{n}\right) \in \mathbb{Z}^{n}, \quad|\gamma|=0
$$

then the value of Kostant's partition function $K_{\left(1^{n}\right)}(\gamma ; 1)$ is equal to the number of $n$ by $n$ skew-symmetric integer matrices $m=\left(m_{i j}\right)_{1 \leq i, j \leq n}$ with row sums $\gamma_{i}$ and column sums $-\gamma_{j}$, such that $m_{i j} \geq 0$, if $i \leq j$. We denote by $S M(\gamma)$ the set of all such skew-symmetric matrices. It is natural to ask does there exist an analog of the Robinson-Schensted-Knuth correspondence for the set of "skew-symmetric" transportation matrices $S M(\gamma)$ ? We don't know any answer on this question in general, but in a particular case when

$$
\gamma=(d, d+1, \ldots, d+n-1,-n(2 d+n-1) / 2),
$$

we suggest such a bijection, see (7.22), Exercise 2c to Section 7. We show also (Exercise $2 \mathbf{h}$ to Section 0 ) that the Ehrhart polynomial $\mathcal{E}\left(C R_{n} ; t\right)$ of the Chan-Robbins polytope $C R_{n}$ is equal to the "continuous analog" $\mathcal{P}_{\Phi(\eta)}(\beta)$ of the Kostant partition function $K_{\Phi(\eta)}(\beta, 1)$. Namely,

$$
\mathcal{E}\left(C R_{n} ; t\right)=\mathcal{P}_{\Phi\left(1^{n+1}\right)}(t, \underbrace{0, \ldots, 0}_{n-1},-t) .
$$

Using a "fermionic formula" for the Kostant partition function and its continuous analog in the dominant chamber $Y_{n+1}^{+}$obtained in [36], we had computed the leading coefficients of the Ehrhart polynomial $\mathcal{E}\left(C R_{n} ; t\right)$, and hence, the volume of Chan-Robbins' polytope $C R_{n}$. Formula for the volume of Chan-Robbins polytope was conjectured in [7], and has been proved for the first time by D. Zeilberger [72]. In Exercise 4 we state a generalized saturation conjecture, and discuss connections between the saturation conjecture (now a theorem by A. Knutson and T. Tao, J. Amer. Math. Soc. 12 (1999), 1055-1090) and parabolic Kostka polynomials. More precisely, for any two partitions $\lambda$ and $\mu$, and a composition $\eta$ such that $|\eta| \geq l(\mu)$ we define numbers $a(\lambda, \mu ; \eta)$ and $b(\lambda, \mu ; \eta)$ via the decomposition

$$
K_{\lambda \mu \eta}(q)=q^{a(\lambda, \mu ; \eta)} b(\lambda, \mu ; \eta)+\text { higher degree terms. }
$$

The numbers $a(\lambda, \mu ; \eta)$ and $b(\lambda, \mu ; \eta)$ seem to have many interesting combinatorial properties. For example, the Littlewood-Richardson numbers $c_{\lambda, \mu}^{\nu}$ are a special case of the numbers $b(\lambda, \mu ; \eta)$, see Section 3, Example 3. In Example 4 (to Section 7) we state several conjectures about the numbers $a(\lambda, \mu ; \eta)$ and $b(\lambda, \mu ; \eta)$. The basic two are:

- generalized saturation conjecture:

$a(n \lambda, n \mu ; \eta)=n a(\lambda, \mu ; \eta)$ for any positive integer $n$;

- generalized Fulton's conjecture:

if $b(n \lambda, n \mu ; \eta)=1$ for some positive integer $n$, then $b(N \lambda, N \mu ; \eta)=1$ for any positive integer $N$. 
In Section 8 we study the limiting behaviour of certain Kostka-Foulkes polynomials. We give a generalization, see Theorem 8.3, of a result obtained by Stanley 64, and give answers on some questions had been posed in [35].

Each Section, except for the first one, ends with a series of exercises (which total number exceeds one hundred). The exercises vary in difficulty: some are straightforward applications of the material presented, while others are more difficult and represent a brief exposition of original works or taken from various papers written by the author.

The bibliographic notes are kept very brief. They are not intended to give a comprehensive historical account or a complete bibliography of the respective subject areas.

\section{Notation}

Throughout the paper we follow to Macdonald's book [52 as for notation related to the theory of symmetric functions, and Stanley's book [66 as for notation related to Combinatorics. Below we give a list of some special notation which we will use

1) if $P(q)$ and $Q(q)$ are polynomials in $q$, the symbol $P(q) \doteq Q(q)$ means that the ratio $P(q) / Q(q)$ is a power of $q$;

2 ) if $a, k_{0}, \ldots, k_{m}$ are (non-negative) integers, the symbol $q^{a}\left(k_{0}, \ldots, k_{m}\right)$ stands for polynomial $\sum_{j=0}^{m} k_{j} q^{a+j}$

3) (Garsia's symbol $\chi(P)$ ) if $P$ is any statement, then $\chi(P)=1$ if it is true, and $\chi(P)=0$ otherwise;

4) if $\lambda$ is a partition and $\mu$ is a composition, the symbol $\operatorname{STY}(\lambda, \mu)$ denotes the set of semistandard Young tableaux of shape $\lambda$ and weight (or content) $\mu$.

\section{Kostka-Foulkes polynomials and weight multiplici- ties}

\subsection{Kostka-Foulkes polynomials}

Kostka-Foulkes polynomials are defined as the matrix elements of the transition matrix

$$
K(q)=M(s, P)
$$

from the Schur functions $s_{\lambda}(x)$ to the Hall-Littlewood functions $P_{\mu}(x ; q)$ :

$$
s_{\lambda}(x)=\sum_{\mu} K_{\lambda \mu}(q) P_{\mu}(x ; q) .
$$

It is well known [52], Chapter I, that if $\lambda$ and $\mu$ partitions, then

- $K_{\lambda \mu}(q) \neq 0$ if and only if $\lambda \geq \mu$ with respect to the dominance partial ordering " $\geq$ " on the set of partitions $\mathcal{P}_{n}$ :

$$
\begin{aligned}
& \lambda \geq \mu \text { if and only if } \\
& \text { i) }|\lambda|=|\mu|, \\
& \text { ii) } \lambda_{1}+\cdots+\lambda_{i} \geq \mu_{1}+\cdots+\mu_{i} \text { for all } i \geq 1 \text {. }
\end{aligned}
$$


- If $\lambda \geq \mu, K_{\lambda \mu}(q)$ is a monic of degree $n(\mu)-n(\lambda)$ polynomial with non-negative integer coefficients.

Let us recall that for any partition $\lambda=\left(\lambda_{1}, \lambda_{2}, \cdots, \lambda_{p}\right)$,

$$
n(\lambda)=\sum_{i=1}^{p}(i-1) \lambda_{i}=\sum_{1 \leq i<j \leq p} \min \left(\lambda_{i}, \lambda_{j}\right) .
$$

It was H.O. Foulkes [12] who asked for a combinatorial explanation of the positivity of coefficients of polynomials $K_{\lambda \mu}(q)$. He conjectured the existence of natural statistics $c(T)$ on the set of semistandard Young tableaux $\operatorname{STY}(\lambda, \mu)$ of shape $\lambda$ and weight $\mu$ such that

$$
K_{\lambda \mu}(q)=\sum_{T \in S T Y(\lambda, \mu)} q^{c(T)} .
$$

This conjecture was settled by Lascoux and Schützenberger [44, 63], who identified $c(T)$ to be the rank of a poset structure on the set $\operatorname{STY}(\bullet, \mu)$ of semistandard Young tableaux of fixed weight $\mu$. Another proof of the positivity of coefficients of polynomials $K_{\lambda \mu}(q)$ has been obtained by Lusztig [48], who identified the polynomials

$$
\widetilde{K}_{\lambda \mu}(q):=q^{n(\mu)-n(\lambda)} K_{\lambda \mu}\left(q^{-1}\right)
$$

with the certain Kazhdan-Lusztig polynomials $P_{n_{\lambda}, n_{\mu}}(q)$ for the affine Weyl group $W\left(\widehat{\mathfrak{g}} l_{n}\right)$.

\subsection{Skew Kostka-Foulkes polynomials}

Let $\lambda, \mu$ and $\nu$ be partitions, $\lambda \supset \mu$, and $|\lambda|=|\mu|+|\nu|$.

Definition 2.1 The skew Kostka-Foulkes polynomials $K_{\lambda \backslash \mu, \nu}(q)$ are defined as the transition coefficients from the skew Schur functions $s_{\lambda \backslash \mu}(x)$ to the Hall-Littlewood functions $P_{\nu}(x ; q)$ :

$$
s_{\lambda \backslash \mu}(x)=\sum_{\nu} K_{\lambda \backslash \mu, \nu}(q) P_{\nu}(x ; q) .
$$

It is clear that

$$
K_{\lambda \backslash \mu, \nu}(q)=\sum_{\pi} c_{\mu \pi}^{\lambda} K_{\pi \nu}(q),
$$

where the coefficients $c_{\mu \pi}^{\nu}=\operatorname{Mult}\left[V_{\nu}: V_{\mu} \otimes V_{\pi}\right]$ stand for the Littlewood-Richardson numbers.

Let us remark that

$$
K_{\lambda \backslash \mu, \nu}(q)=\sum_{T} q^{c(T)}
$$

summed over all semistandard skew tableaux $T$ of shape $\lambda \backslash \mu$ and weight $\nu$, where $c(T)$ denotes the charge of skew tableau $T$.

We define also cocharge version of the skew Kostka-Foulkes polynomials:

$$
\bar{K}_{\lambda \backslash \mu, \nu}(q)=\sum_{\pi} c_{\mu \pi}^{\lambda} \bar{K}_{\pi \mu}(q),
$$

where $\bar{K}_{\lambda \mu}(q)=q^{n(\mu)} K_{\lambda \mu}\left(q^{-1}\right)$. 


\subsection{Weight multiplicities}

Let $V$ be a finite dimensional representation of the Lie algebra $\mathfrak{g} l(n)$, and $\mu$ be an integrable weight (i.e. composition), denote by $V(\mu)$ the weight $\mu$ subspace of the representation $V$. Dimension $\operatorname{dim} V(\mu)$ of the space $V(\mu)$ is called weight multiplicity of $\mu$ in $V$. Let $\operatorname{ch} V$ denote the character of representation $V$. It is a symmetric polynomial in variables $X_{n}=$ $\left(x_{1}, \ldots, x_{n}\right)$. If $P$ is any polynomial in the variables $X_{n}$, let $\left[x^{\mu}\right] P$ be the coefficient of $x^{\mu}=x_{1}^{\mu_{1}} \cdots x_{n}^{\mu_{n}}$ in $P$. It is clear that

$$
\operatorname{dim} V(\mu)=\left[x^{\mu}\right] \operatorname{ch} V
$$

Follow S. Kato [28], define a $q$-analog $\operatorname{dim}_{q} V(\mu)$ of the weight multiplicity $\operatorname{dim} V(\mu)$ as the transition coefficient from the character $\operatorname{ch} V$ to the Hall-Littlewood polynomials $P_{\mu}\left(X_{n} ; q\right)$ :

$$
\operatorname{ch} V=\sum_{\mu} \operatorname{dim}_{q} V(\mu) P_{\mu}\left(X_{n} ; q\right)
$$

In the present paper we are mainly interested in the following two cases:

i) $V=V_{\lambda}$ is an irreducible highest weight $\lambda$ representation of the Lie algebra $\mathfrak{g} l(n)$.

In this case the character $\operatorname{ch} V_{\lambda}$ of representation $V_{\lambda}$ coincides with the Schur function $s_{\lambda}\left(X_{n}\right)$, and dimension $\operatorname{dim} V_{\lambda}(\mu)$ of the weight $\mu$ subspace of $V_{\lambda}$ is equal to the Kostka number

$$
K_{\lambda \mu}:=K_{\lambda \mu}(1)
$$

and is equal also to the number of semistandard Young tableaux of shape $\lambda$ and weight $\mu$. It follows from (2.6) and (2.1) that the $q$-analog $\operatorname{dim}_{q} V_{\lambda}(\mu)$ of weight multiplicity $\operatorname{dim} V_{\lambda}(\mu)$ is equal to the Kostka-Foulkes polynomial $K_{\lambda \mu}(q)$. Polynomials $K_{\lambda \mu}(q)$ and the Lusztig $q$-analog of weight multiplicity $d_{\mu}\left(L_{\lambda} ; q\right)$ in the case of type $A$, see 49, p.215, (6.10), are connected by the following relation

$$
d_{\mu}\left(L_{\lambda} ; q\right)=q^{n(\mu)-n(\lambda)} K_{\lambda \mu}\left(q^{-1}\right)
$$

In the sequel, we will denote the Lusztig polynomials $d_{\mu}\left(L_{\lambda} ; q\right)$ by $\widetilde{K}_{\lambda \mu}(q)$, so that

$$
\widetilde{K}_{\lambda \mu}(q)=q^{n(\mu)-n(\lambda)} K_{\lambda \mu}\left(q^{-1}\right)=q^{-n(\lambda)} \bar{K}_{\lambda \mu}(q) .
$$

ii) $V=V_{(\mu)}=V_{\left(\mu_{1}\right)} \otimes \cdots \otimes V_{\left(\mu_{r}\right)}$ is the tensor product of irreducible representations $V_{\left(\mu_{a}\right)}$ corresponding to the one row partitions $\left(\mu_{a}\right), 1 \leq a \leq r$, and $\mu=\left(\mu_{1}, \ldots, \mu_{r}\right)$.

In this case the character $\operatorname{ch} V_{(\mu)}$ is equal to the product $\prod_{j=1}^{r} h_{\mu_{j}}\left(X_{n}\right)$ of complete homogeneous symmetric polynomials $h_{\mu_{j}}\left(X_{n}\right), 1 \leq j \leq r$. The weight $\lambda$ multiplicity $\operatorname{dim} V_{(\mu)}(\lambda)$ is equal to the number $\mathcal{P}_{\lambda \mu}$ of $n$ by $n$ matrices of non-negative integers with row sums $\lambda_{i}$ and column sums $\mu_{j}$ :

$$
\operatorname{dim} V_{(\mu)}(\lambda)=\sum_{\eta} K_{\eta \mu} K_{\eta \lambda}=\mathcal{P}_{\lambda \mu}
$$


We denote by $\mathcal{P}_{\lambda \mu}$ the set of all $n$ by $n$ matrices of non-negative integers with row sums $\lambda_{i}$ and column sums $\mu_{j}$ (the set of so-called transportation matrices of type $(\lambda ; \mu)$ ). There exists a remarkable bijection, known as the Robinson-Schensted-Knuth correspondence,

$$
\mathcal{P}_{\lambda \mu} \simeq \coprod_{\eta} S T Y(\eta, \mu) \times S T Y(\eta, \lambda)
$$

between the set of transportation matrices of type $(\lambda ; \mu)$ and that of all pairs $(P, Q)$ of semistandard Young tableaux of the same shape and weights $\mu$ and $\lambda$, correspondingly. The Robinson-Schensted-Knuth correspondence (2.9) leads to a combinatorial/bijective proof of the last equality in (2.8).

Finally, let us find the $q$-analog, denoted by $\operatorname{dim}_{q} V_{(\mu)}(\lambda)$, of multiplicity of the weight $\lambda$ in representation $V_{(\mu)}$. Using (2.6), (2.1) and the product formula for the character of representation $V_{(\mu)}$ :

$$
\operatorname{ch} V_{(\mu)}=\prod_{j=1}^{r} h_{\mu_{j}}\left(X_{n}\right)
$$

one can obtain that

$$
\operatorname{dim}_{q} V_{(\mu)}=\sum_{\eta} K_{\eta \mu} K_{\eta \lambda}(q):=\mathcal{P}_{\lambda \mu}(q)
$$

Polynomials $\mathcal{P}_{\lambda \mu}(q)$ admit numerous algebraic, algebro-geometric and combinatorial interpretations. Some of them were mentioned in Introduction. Here we only mention a result by R. Hotta and N. Shimomura (Math. Ann. 24 (1979), 193-198) that the product $t^{n(\lambda)} \mathcal{P}_{\lambda \mu}\left(t^{-1}\right)$ is equal to the Poincare polynomial of the partial unipotent flag variety $\mathcal{F}_{\mu}^{\lambda}$ :

$$
t^{n(\lambda)} \mathcal{P}_{\lambda \mu}\left(t^{-1}\right)=\sum_{k \geq 0} \operatorname{dim} H^{2 k}\left(\mathcal{F}_{\mu}^{\lambda}, \mathbb{Q}\right) t^{k},
$$

see [34, 43, 69] and references therein.

\section{Exercises}

1. Rectangular Catalan and Narayana polynomials, and MacMahon polytope

a. Define rectangular Catalan polynomial

$$
C(n, m \mid q)=\frac{(q ; q)_{n m}}{\prod_{i=1}^{n} \prod_{j=1}^{m}\left(1-q^{i+j-1}\right)}
$$

Show that

$$
q^{m\left(\begin{array}{c}
n \\
2
\end{array}\right)} C(n, m \mid q)=K_{\left(n^{m}\right),\left(1^{n m}\right)}(q) .
$$

Thus, $C(n, m \mid q)$ is a polynomial of degree $n m(n-1)(m-1) / 2$ in the variable $q$ with nonnegative integer coefficients. Moreover,

$$
C(n, 2 \mid q)=C(2, n \mid q)=c_{n}(q)=\frac{1-q}{1-q^{n+1}}\left[\begin{array}{c}
2 n \\
n
\end{array}\right]_{q}
$$


coincides with "the most obvious" $q$-analog of the Catalan numbers, see e.g. [14], p.255, or 66.

b. It follows from (2.12) that the rectangular Catalan number $C(n, m \mid 1)$ counts the number of lattice words

$$
w=a_{1} a_{2} \cdots a_{n m}
$$

of weight $\left(m^{n}\right)$, i.e. lattice words in which each $i$ between 1 and $m$ occurs exactly $n$ times. Let us recall that a word $a_{1} \cdots a_{p}$ in the symbols $1, \ldots, m$ is said to be a lattice word, if for $1 \leq r \leq p$ and $1 \leq j \leq m-1$, the number of occurrences of the symbol $j$ in $a_{1} \cdots a_{r}$ is not less than the number of occurrences of $j+1$ :

$$
\#\left\{i \mid 1 \leq i \leq r \text { and } a_{i}=j\right\} \geq \#\left\{i \mid 1 \leq i \leq r \text { and } a_{i}=j+1\right\} .
$$

For any word $w=a_{1} \cdots a_{k}$, in which each $a_{i}$ is a positive integer, define the major index

$$
\operatorname{maj}(w)=\sum_{i=1}^{k-1} i \chi\left(a_{i}>a_{i+1}\right)
$$

and the number of descents

$$
\operatorname{des}(w)=\sum_{i=1}^{k-1} \chi\left(a_{i}>a_{i+1}\right)
$$

Finally, for any integer $k$ between 0 and $(n-1)(m-1)$, define rectangular $q-N a r a y a n a$ number

$$
N(n, m ; k \mid q)=\sum_{w} q^{\operatorname{maj}(w)},
$$

where $w$ ranges over all lattice words of weight $\left(m^{n}\right)$ such that $\operatorname{des}(w)=k$.

Example 2.2 Take $n=4, m=3$, then

$$
\sum_{k=0}^{6} N(3,4 ; k \mid 1) t^{k}=1+22 t+113 t^{2}+190 t^{3}+113 t^{4}+22 t^{5}+t^{6}
$$

This example shows that if $n, m \geq 3$, it is unlikely that there exists a simple combinatorial formula for the rectangular Narayana numbers $N(n, m ; k \mid 1)$, but see formula (2.14).

- Show that

i) $C(n, m \mid q)=\sum_{w} q^{\operatorname{maj}(w)}$, where $w$ ranges over all lattice words of weight $\left(m^{n}\right)$;

ii) $N(n, m ; k \mid q)=q^{n m((n-1)(m-1) / 2-k)} N(n, m ;(n-1)(m-1)-k \mid q)=N(m, n ; k \mid q)$, for any integer $k, 0 \leq k \leq(n-1)(m-1) / 2$;

iii) $N(2, n ; k \mid q)=q^{k(k+1)} \frac{1-q}{1-q^{n}}\left[\begin{array}{l}n \\ k\end{array}\right]_{q}\left[\begin{array}{c}n \\ k+1\end{array}\right]_{q} \doteq \operatorname{dim}_{q} V_{(k, k)}^{\mathfrak{g l}(n-k+1)}$, for any integer $k$, $0 \leq k \leq n-1$, where $V_{(k, k)}^{\mathfrak{g l}(n-k+1)}$ stands for the irreducible representation of the Lie algebra 
$\mathfrak{g} l(n-k+1)$ corresponding to the two row partition $(k, k)$; recall that for any finite dimensional $\mathfrak{g} l(N)$-module $V$ the symbol $\operatorname{dim}_{q} V$ denotes its $q$-dimension, i.e. the principal specialization of the character of the module $V$ :

$\operatorname{dim}_{q} V=(\operatorname{ch} V)\left(1, q, \ldots, q^{N-1}\right)$

iv) $N(n, m ; 1 \mid 1)=\sum_{j \geq 2}\left(\begin{array}{c}n \\ j\end{array}\right)\left(\begin{array}{c}m \\ j\end{array}\right)=\left(\begin{array}{c}n+m \\ n\end{array}\right)-n m-1$;

v) (Fermionic formula for $q$-Narayana numbers)

$$
q^{m\left(\begin{array}{c}
n \\
2
\end{array}\right)} N(n, m ; l \mid q)=\sum_{\{\nu\}} q^{c(\nu)} \prod_{k, j \geq 1}\left[\begin{array}{c}
P_{j}^{(k)}(\nu)+m_{j}\left(\nu^{(k)}\right) \\
m_{j}\left(\nu^{(k)}\right)
\end{array}\right]_{q},
$$

summed over all sequences of partitions $\{\nu\}=\left\{\nu^{(1)}, \nu^{(2)}, \ldots, \nu^{(m-1)}\right\}$ such that

- $\left|\nu^{(k)}\right|=(m-k) n, 1 \leq k \leq m-1$;

- $\left(\nu^{(1)}\right)_{1}^{\prime}=(m-1) n-l$, i.e. the length of the first column of the diagram $\nu^{(1)}$ is equal to $(m-1) n-l$

- $P_{j}^{(k)}(\nu):=Q_{j}\left(\nu^{(k-1)}\right)-2 Q_{j}\left(\nu^{(k)}\right)+Q_{j}\left(\nu^{(k+1)}\right) \geq 0$, for all $k, j \geq 1$,

where by definition we put $\nu^{(0)}=\left(1^{n m}\right)$; for any diagram $\lambda$ the number $Q_{j}(\lambda)=\lambda_{1}^{\prime}+\cdots \lambda_{j}^{\prime}$ is equal to the number of cells in the first $j$ columns of the diagram $\lambda$, and $m_{j}(\lambda)$ is equal to the number of parts of $\lambda$ of size $j$;

$$
\text { - } c(\nu)=\sum_{k, j \geq 1}\left(\begin{array}{c}
\left(\nu^{(k-1)}\right)_{j}^{\prime}-\left(\nu^{(k)}\right)_{j}^{\prime} \\
2
\end{array}\right) \text {. }
$$

Conjecture 2.3 If $1 \leq k \leq(n-1)(m-1) / 2$, then

$$
N(n, m ; k-1 \mid 1) \leq N(n, m ; k \mid 1),
$$

i.e. the sequence of rectangular Narayana numbers $\{N(n, m ; k \mid 1)\}_{k=0}^{(n-1)(m-1)}$ is symmetric and unimodal.

For definition of unimodal polynomials/sequences see e.g. 65, where one may find a big variety of examples of unimodal sequences which frequently appear in Algebra, Combinatorics and Geometry.

c. (Volume of the MacMahon polytope) Let $\mathfrak{M}_{m n}$ be the convex polytope in $\mathbb{R}^{n m}$ of all points $\mathbf{x}=\left(x_{i j}\right)_{1 \leq i \leq n, 1 \leq j \leq m}$ satisfying the following conditions

$$
0 \leq x_{i j} \leq 1, \quad x_{i j} \geq x_{i-1, j}, \quad x_{i j} \geq x_{i, j-1},
$$

for all pairs of integers $(i, j)$ such that $1 \leq i \leq n, 1 \leq j \leq m$, and where by definition we set $x_{i 0}=0=x_{0 j}$.

We will call the polytope $\mathfrak{M}_{n m}$ by MacMahon polytope. The MacMahon polytope is an integral polytope of dimension $n m$ with $\left(\begin{array}{c}m+n \\ n\end{array}\right)$ vertices which correspond to the set of $(0,1)$-matrices satisfying (2.15). 
If $k$ is a positive integer, define $i\left(\mathfrak{M}_{n m} ; k\right)$ to be the number of points $\mathbf{x} \in \mathfrak{M}_{n m}$ such that $k \mathbf{x} \in \mathbb{Z}^{n m}$. Thus, $i\left(\mathfrak{M}_{n m} ; k\right)$ is equal to the number of plane partitions of rectangular shape $\left(n^{m}\right)$ with all parts do not exceed $k$. By a theorem of MacMahon (see e.g. [52, Chapter I, $\S 5$, Example 13)

$$
i\left(\mathfrak{M}_{n m} ; k\right)=\prod_{i=1}^{n} \prod_{j=1}^{m} \frac{k+i+j-1}{i+j-1} .
$$

It follows from (2.16) that the Ehrhart polynomial $\mathcal{E}\left(\mathfrak{M}_{n m} ; t\right)$ of the MacMahon polytope $\mathfrak{M}_{n m}$ is completely resolved into linear factors:

$$
\mathcal{E}\left(\mathfrak{M}_{n m} ; t\right)=\prod_{i=1}^{n} \prod_{j=1}^{m} \frac{t+i+j-1}{i+j-1} .
$$

Hence, the normalized volume

$$
\widetilde{\operatorname{vol}}\left(\mathfrak{M}_{n m}\right)=(n m) ! \operatorname{vol}\left(\mathfrak{M}_{n m}\right)
$$

of the MacMahon polytope $\mathfrak{M}_{n m}$ is equal to the rectangular Catalan number $C(n, m \mid 1)$, i.e. the number of standard Young tableaux of rectangular shape $\left(n^{m}\right)$. We refer the reader to [66], Section 4.6, and [25, Chapter IX, for definition and basic properties of the Ehrhart polynomial $\mathcal{E}(\mathcal{P} ; t)$ of a convex integral polytope $\mathcal{P}$.

- Show that

$$
\sum_{k \geq 0} i\left(\mathfrak{M}_{n m} ; k\right) z^{k}=\left(\sum_{j=0}^{(n-1)(m-1)} N(n, m ; j) z^{j}\right) /(1-z)^{n m+1},
$$

where

$$
N(n, m ; j):=N(n, m ; j \mid 1)
$$

denotes the rectangular Narayana number. Thus, the sequence of Narayana numbers

$$
(1=N(n, m ; 0), N(n, m ; 1), \ldots, N(n, m ;(n-1)(m-1))=1)
$$

is the $\delta$-vector (see e.g. [25], §34) of the MacMahon polytope. In the case $n=2$ (or $m=2$ ) all these results may be found in [66], Chapter 6, Exercise 6.31.

Questions. i) (Higher associahedron) Does there exist an $(m-1)(n-1)$-dimensional integral convex (simplicial?) polytope $Q_{n, m}$ which has $h$-vector

$$
h=\left(h_{0}\left(Q_{n, m}\right), h_{1}\left(Q_{n, m}\right), \ldots, h_{(n-1)(m-1)}\left(Q_{n, m}\right)\right)
$$

given by

$$
\sum_{i=0}^{(n-1)(m-1)} h_{i}\left(Q_{n, m}\right) q^{(n-1)(m-1)-i}=C(n, m \mid q) ?
$$


We refer the reader to [25], Chapter I, $\S 6$ and Chapter III, for definitions and basic properties of the $h$-vector of a simplicial polytope; see also, R. Stanley (J. Pure and Appl. Algebra 71 (1991), 319-331).

ii) Define rectangular Schröder polynomial

$$
S(n, m \mid q):=C(n, m \mid 1+q)
$$

and put

$$
S(n, m \mid q)=\sum_{k \geq 0}^{(n-1)(m-1)} S(n, m \| k) q^{k} .
$$

What is a combinatorial interpretation(s) of the numbers $S(n, m \| k)$ and $S(n, m \mid 1)$ ?

The answers on these questions are known if either $n$ or $m$ is equal to 2 , see e.g. R. Simion (Adv. in Appl. Math. 18 (1997), 149-180, Example 4 (the Associahedron)). Note finally, that MacMahon's polytope and Narayana's numbers will appear again in Section 07, Exercise $3 \mathbf{e}$.

d. Denote by $f(n, d)$ the number of ways to draw $d$ diagonals in a convex $(n+2)$-gon, such that no two diagonals intersect in their interior.

i) Show that

$$
f(n, d)=\frac{1}{n+1}\left(\begin{array}{c}
n+d+1 \\
d+1
\end{array}\right)\left(\begin{array}{c}
n-d \\
d
\end{array}\right)=K_{\left((d+1)^{2}, 1^{n-d-1}\right),\left(1^{n+d+1}\right)}(1) .
$$

For example, $f(n, n-1)$ is just the Catalan number

$$
C_{n}=\frac{1}{n+1}\left(\begin{array}{c}
2 n \\
n
\end{array}\right)
$$

The first equality in the formula (2.18) goes back to T.K. Kirkman (1857), E. Prouhet (1866), and A. Cayley (1890); for precise references, see [66], p.272.

It follows from (2.18) that $f(n, d)$ is equal to the number of standard Young tableaux of shape $\left((d+1)^{2}, 1^{n-d-1}\right)$. It is natural to ask for a bijection between the polygon dissections and standard Young tableaux. Such a bijection was constructed by R. Stanley (J. Comb. Theory A, 76 (1996), 175-177).

ii) Define a statistics $b$ on the set $F(n, d)$ of $d$-tuples of non-crossing in their interior diagonals in a convex $(n+2)$-gon such that

$$
\sum_{f \in F(n, d)} q^{b(f)}=\widetilde{K}_{\left((d+1)^{2}, 1^{n-d-1}\right),\left(1^{n+d+1}\right)}(q) .
$$

2. Let $\mu$ be a composition, $|\mu|=n$.

a. Show that

$$
\mathcal{P}_{\left(1^{n}\right), \mu}(q)=\sum_{\eta} K_{\eta \mu} K_{\eta,\left(1^{n}\right)}(q)=q^{n\left(\mu^{\prime}\right)}\left[\begin{array}{c}
n \\
\mu_{1}, \ldots, \mu_{n}
\end{array}\right]_{q} .
$$


Formula (2.19) goes back to C. Ehresmann who had computed (Ann. Math. 35 (1934), 396-443) the Poincare polynomial of the partial flag variety $\mathcal{F}_{\mu}$ corresponding to a composition $\mu$ of $n$ :

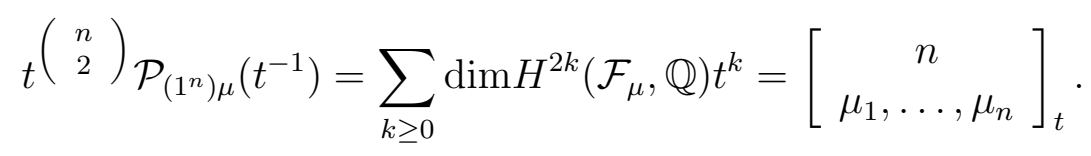

b. (A generalization of the previous Exercise) Let $R=\left\{\left(\mu_{a}^{\eta_{a}}\right)\right\}_{a=1}^{l}$ be a sequence of rectangular shape partitions, $N=\sum_{a=1}^{l} \mu_{a} \eta_{a}$. Show that

$$
\sum_{\lambda} K_{\lambda R} K_{\lambda,\left(1^{N}\right)}(q)=\prod_{a=1}^{l} K_{\left(\mu_{a}^{n_{a}}\right),\left(1^{\left.\mu_{a} \eta_{a}\right)}\right.}(q)\left[\begin{array}{c}
N \\
\mu_{1} \eta_{1}, \ldots, \mu_{l} \eta_{l}
\end{array}\right]_{q},
$$

where $K_{\lambda R}$ denotes the value of parabolic Kostka polynomial $K_{\lambda R}(q)$ at $q=1$, see Section of, Definition 3.7. In particular, if sequence

$$
R=\left\{\mu_{1}, \ldots, \mu_{r}, 1^{\eta_{1}}, \ldots, 1^{\eta_{s}}\right\}
$$

consists of only one row or one column partitions, and

$$
\sum_{j=1}^{r} \mu_{j}+\sum_{j=1}^{s} \eta_{j}=N
$$

then

$$
\sum_{\lambda} K_{\lambda R} K_{\lambda,\left(1^{N}\right)}(q)=q^{n\left(\mu^{\prime}\right)}\left[\begin{array}{c}
N \\
\mu_{1}, \ldots, \mu_{r}, \eta_{1}, \ldots, \eta_{s}
\end{array}\right]_{q} .
$$

3. Define polynomial $\varphi_{\nu \mu}^{\lambda}(q)$ to be the coefficient of $P_{\lambda}$ in the product $s_{\nu} P_{\mu}$ :

$$
s_{\nu}(x) P_{\mu}(x ; q)=\sum_{\lambda} \varphi_{\nu \mu}^{\lambda}(q) P_{\lambda}(x ; q) .
$$

Equivalently,

$$
\varphi_{\nu \mu}^{\lambda}(q)=\sum_{\eta} K_{\nu \eta}(q) f_{\eta \mu}^{\lambda}(q),
$$

where for any three partitions $\lambda, \mu, \nu$ the polynomial $f_{\mu \nu}^{\lambda}(q)$ is the coefficient of $P_{\lambda}$ in the product $P_{\mu} P_{\nu}$, see e.g. [52], Chapter III, $\S 3$. Recall that $P_{\lambda}, P_{\mu}, \ldots$ denote the Hall-Littlewood polynomials corresponding to partitions $\lambda, \mu, \ldots$.

Conjecture 2.4 For any three partitions $\lambda, \mu, \nu$ the polynomial $\varphi_{\nu \mu}^{\lambda}(q)$ is a polynomial with non-negative integer coefficients.

For example:

$$
\varphi_{\left(1^{n}\right) \mu}^{\lambda}(q)=f_{\left(1^{n}\right) \mu}^{\lambda}(q)=\prod_{i \geq 1}\left[\begin{array}{c}
\lambda_{i}^{\prime}-\lambda_{i+1}^{\prime} \\
\lambda_{i}^{\prime}-\mu_{i}^{\prime}
\end{array}\right]_{q},
$$


and therefore $\varphi_{\left(1^{n}\right) \mu}^{\lambda}(q)=0$, unless $\lambda \backslash \mu$ is a vertical $n$-strip, [52], Chapter III, (3.2), p.215;

$$
\left.\varphi_{(n) \mu}^{\lambda}(q)=q^{c(\lambda ; \mu)} \prod_{i \geq 1}\left[\begin{array}{c}
\lambda_{i}^{\prime}-\mu_{i+1}^{\prime} \\
\lambda_{i}^{\prime}-\mu_{i}^{\prime}
\end{array}\right]_{q}, \quad c_{(} \lambda ; \mu\right)=\sum_{i \geq 0}\left(\begin{array}{c}
\lambda_{i}^{\prime}-\mu_{i}^{\prime} \\
2
\end{array}\right)
$$

and therefore

$$
\varphi_{(n) \mu}^{\lambda}(q)=0
$$

unless $\mu \subset \lambda,|\lambda \backslash \mu|=n,[34,26]$.

Perhaps, it is interesting to mention that if $p$ is a prime number, then

$$
\varphi_{(n) \mu}^{\lambda}(p)=p^{n(\lambda)-n(\mu)} \alpha_{\lambda}\left(\mu ; p^{-1}\right),
$$

where for any partitions $\mu \subseteq \lambda$ we denote by $\alpha_{\lambda}(\mu ; p)$ the number of subgroups of type $\mu$ in a finite abelian $p$-group of type $\lambda$, see [5] and [34] for definitions and further details.

a. Based on (2.24), prove the following combinatorial/fermionic formula for the $q$-weight multiplicity $\mathcal{P}_{\lambda \mu}(q):=\operatorname{dim}_{q} V_{(\mu)}(\lambda)$ :

$$
\mathcal{P}_{\lambda \mu}(q)=\sum_{\eta} K_{\eta \mu} K_{\eta \lambda}(q)=\sum_{\{\nu\}} q^{c(\nu)} \prod_{k=1}^{r-1} \prod_{i \geq 1}\left[\begin{array}{c}
\nu_{i}^{(k+1)}-\nu_{i+1}^{(k)} \\
\nu_{i}^{(k)}-\nu_{i+1}^{(k)}
\end{array}\right]_{q}
$$

summed over all flags of partitions $\{\nu\}=\left\{0=\nu^{(0)} \subset \nu^{(1)} \subset \cdots \subset \nu^{(r)}=\lambda^{\prime}\right\}$, such that $\left|\nu^{(k)}\right|=\mu_{1}+\cdots+\mu_{k}$, for all $k, 1 \leq k \leq r=l(\mu)$;

$$
c(\nu)=\sum_{k=0}^{r-1} \sum_{i \geq 1}\left(\begin{array}{c}
\nu_{i}^{(k+1)}-\nu_{i}^{(k)} \\
2
\end{array}\right)
$$

where $\lambda^{\prime}$ denotes the conjugate of the partition $\lambda$, see e.g. [52, Chapter I, p.2, and for any real number $x$ we put

$$
\left(\begin{array}{l}
x \\
2
\end{array}\right):=x(x-1) / 2 .
$$

For a proof and applications of (2.25) see [34, 26].

Note also a preprint by G. Lusztig, Fermionic form and Betti numbers, math.QA/0005010, where a conjectural formula for the Betti numbers of a lagrangian quiver variety (see H. Nakajima, Duke Math. J. 91 (1998), 515-560, for definitions), which generalizes the first equality in (2.25), is suggested. It seems a very challenging task to find for lagrangian quiver variety (constructed by H. Nakajima [ibid] and G. Lusztig [ibid]) an analog of the second equality in (2.25).

b. Consider the transition matrix

$$
M(e, P):=\left(M(e, P)_{\lambda \mu}\right)
$$

which expresses the elementary symmetric polynomials $e_{\lambda}$ (see e.g. [52, Chapter I, §2) in terms of Hall-Littlewood polynomials:

$$
e_{\lambda}\left(X_{n}\right)=\sum_{\mu} M(e, P)_{\lambda \mu} P_{\mu}\left(X_{n} ; q\right) .
$$


It is well-known ([ibid], Chapter III, §6) that

$$
M(e, P)_{\lambda \mu}=\sum_{\eta} K_{\eta^{\prime} \lambda} K_{\eta \mu}(q)
$$

- Use (2.23) to derive the following combinatorial formula for the transition coefficients between the elementary symmetric and Hall-Littlewood polynomials:

$$
M(e, P)_{\lambda \mu}=\sum_{\{\nu\}} \prod_{k=1}^{n-1} \prod_{i \geq 1}\left[\begin{array}{c}
\nu_{i}^{(k+1)}-\nu_{i+1}^{(k+1)} \\
\nu_{i}^{(k)}-\nu_{i+1}^{(k+1)}
\end{array}\right]_{q}
$$

summed over all flags of partitions $\{\nu\}:=\left\{0=\nu^{(0)} \subset \nu^{(1)} \subset \cdots \subset \nu^{(r)}=\lambda^{\prime}\right\}$ such that $\nu^{(k)} / \nu^{(k-1)}$ is a horizontal strip of length $\mu_{k}, 1 \leq k \leq r=l(\mu)$.

For a proof and applications of (2.26) see [34, 26].

Remark 2.5 The specialization $q=1$ in the both sides of formula $(2.26)$ gives rise to a combinatorial formula for the number of $(0,1)$-matrices with the prescribed row sums $\lambda_{i}$ and column sums $\mu_{j}$. The latter set (denoted by $\mathcal{R}_{\lambda \mu}$ ) has drawn considerable attention among the specialists in combinatorics and geometry, see e.g., survey articles by R.A. Brualdi (Linear Algebra Appl. 33 (1980), 159-231), and by G. Ziegler (in Polytopes-Combinatorics and Computations, G. Kalai and G. Ziegler (eds.), Birkhäuser, 2000, 1-43). A problem of finding the precise number of matrices in the set $\mathcal{R}_{\lambda \mu}$ has been discussed frequently in the literature, see e.g., R.J. Ryser (Bull. Amer. Math. Soc. 66 (1960), 442-464).

As far as I am aware, for the first time such a formula was obtained by B.Y. Wang (Scienta Sinica (Series A) 1 (1988), 1-6) and simplified later by B.Y. Wang and F. Zhang (Discr. Math. 187 (1998), 211-220). The formula for cardinality of the set $\mathcal{R}_{\lambda \mu}$ obtained by B.Y. Wang and F. Zhang, involves $2^{n-1}-n$ summations and (probably) does not have a natural $q$-analog which is compatible with the dual Knuth correspondence (see e.g., D. Knuth, Pacific J. Math. 34 (1970), 709-727) and the Lascoux-Schützenberger statistics charge, see e.g. 52], Chapter III, §6. It is unclear for the author are there some relations between the Wang-Zhang formula for the number $\left|\mathcal{R}_{\lambda \mu}\right|$ and that (2.26). Finally, note that yet another combinatorial formula for the cardinality of the set $\mathcal{R}_{\lambda \mu}$ follows from Theorem 7.14 .

4. (Generalized Rogers-Szegö and modified Hall-Littlewood polynomials)

a. The homogeneous Rogers-Szegö polynomial $H_{N}(x, y ; q)$ is defined by

$$
H_{N}(x, y ; q)=\sum_{k=0}^{N}\left[\begin{array}{c}
N \\
k
\end{array}\right]_{q} x^{k} y^{N-k}
$$

This polynomial can be viewed as a $q$-deformation of homogeneous Hermite polynomial $H_{N}(x, y)$. Let us recall the generating function for the Hermite polynomials

$$
\sum_{N \geq 0} \frac{H_{N}(x, y)}{N !} t^{N}=\exp \left(2 x t-y^{2} t^{2}\right)
$$


see e.g., G. Andrews, R. Askey and R. Roy, Special Functions, Cambridge Univ. Press, New York, 1999, Chapter 6.

- Show that generating function for the Rogers-Szegö polynomials $H_{N}(x, y ; q)$ is given by

$$
\sum_{N \geq 0} \frac{H_{N}(x, y ; q)}{(q ; q)_{N}} t^{N}=\frac{1}{(t x ; q)_{\infty}(t y ; q)_{\infty}} .
$$

- Use (2.27) to derive the following recurrence relation for Rogers-Szegö polynomials

$$
H_{N}(x, y ; q)=(x+y) H_{N-1}(x, y ; q)-\left(1-q^{N-1}\right) x y H_{N-2}(x, y ; q) .
$$

b. More generally, follow [四, Chapter 3, Example 17, let us define the generalized RogersSzegö polynomial

$$
H_{N}\left(X_{n} ; q\right)=\sum_{k_{1}+\cdots+k_{n}=N}\left[\begin{array}{c}
N \\
k_{1}, \ldots, k_{n}
\end{array}\right]_{q} x_{1}^{k_{1}} \cdots x_{n}^{k_{n}} .
$$

- Use (1.3) and (2.19) to derive that

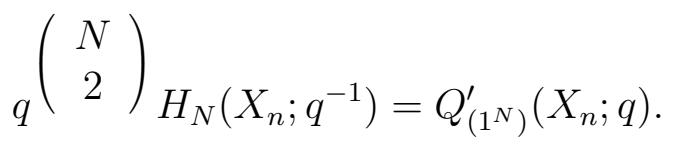

Recall that $X_{n}:=\left(x_{1}, \ldots, x_{n}\right)$ stands for the set of variables, and $Q_{\mu}^{\prime}\left(X_{n} ; q\right)$ denotes the modified Hall-Littlewood polynomial corresponding to partition $\mu$.

- Show that the generating function for generalized Rogers-Szegö polynomials is given by

$$
\sum_{N \geq 0} \frac{H_{N}\left(X_{n} ; q\right)}{(q ; q)_{N}} t^{N}=\prod_{j=1}^{n} \frac{1}{\left(t x_{j} ; q\right)_{\infty}}
$$

Based on (2.29), deduce the following recurrence relation for generalized Rogers-Szegö polynomials

$$
H_{N}\left(X_{n} ; q\right)=\sum_{k=1}^{n}(-1)^{k-1} \frac{(q ; q)_{N-1}}{(q ; q)_{N-k}} e_{k}\left(X_{n}\right) H_{N-k}\left(X_{n} ; q\right)
$$

where $e_{k}\left(X_{n}\right)$ stands for the elementary symmetric function of degree $k$ in the variables $X_{n}$.

Perhaps, it is interesting to mention that the specialization

$$
\begin{aligned}
Z_{N}^{(n)}(q) & :=q^{-N(N-n) / 2 n} Q_{\left(1^{N}\right)}^{\prime}\left(x_{1}=1, \ldots, x_{n}=1 ; q\right) \\
& =q^{-N(N-n) / 2 n} \sum_{\lambda \vdash N} K_{\lambda,\left(1^{N}\right)}(q) \operatorname{dim} V_{\lambda}^{\mathfrak{g} l(N)}
\end{aligned}
$$

is equal to the partition function for the so-called $S U(n)$ Polychronakos-Frahm model, defined by the Hamiltonian

$$
\mathcal{H}_{P F}=\sum_{1 \leq j<k \leq N} \frac{P_{j k}}{\left(t_{j}-t_{k}\right)^{2}}
$$


where $P_{j k}$ denotes the so-called exchange operator in $s u(n)$ spin space, and $\left\{t_{j}\right\}$ are the roots of the $N$-th order (inhomogeneous) Hermite polynomial

$$
H_{N}(t)=y^{-N} H_{N}(x, y), \quad t=x / y \text {. }
$$

For more details, see K. Hikami, J. Phys. Soc. Japan 64 (1995), 1047-1050, and the literature quoted therein.

\section{$3 \quad$ Parabolic Kostka polynomials}

Let $X_{n}=\left(x_{1}, \cdots, x_{n}\right)$ be the set of independent variables. For any sequence of integers $\gamma=\left(\gamma_{1}, \ldots, \gamma_{n}\right)$ put

$$
x^{\gamma}=x_{1}^{\gamma_{1}} x_{2}^{\gamma_{2}} \cdots x_{n}^{\gamma_{n}} .
$$

The symmetric group $S_{n}$ acts on polynomials and rational functions in $X_{n}=\left(x_{1}, \ldots, x_{n}\right)$ by permuting variables.

Define operators

$$
\begin{aligned}
J(f) & =\sum_{w \in S_{n}}(-1)^{l(w)} w\left(x^{\delta} f\right), \\
\pi_{n}(f) & =J(1)^{-1} J(f),
\end{aligned}
$$

where

$$
J(1)=\prod_{1 \leq i<j \leq n}\left(x_{i}-x_{j}\right)
$$

is the Vandermond determinant, and $\delta:=\delta_{n}=(n-1, n-2, \ldots, 1,0)$.

Examples $3.11^{0}$ For the dominant (i.e. weakly decreasing) integral weight $\lambda=\left(\lambda_{1} \geq \lambda_{2} \geq\right.$ $\left.\cdots \geq \lambda_{n}\right)$, the character $\operatorname{chV}_{\lambda}$ of the irreducible highest weight $\lambda \mathfrak{g l}(n)$-module $V_{\lambda}$ is given by the Laurent polynomial

$$
s_{\lambda}\left(X_{n}\right)=\pi_{n}\left(x^{\lambda}\right)=\operatorname{chV}_{\lambda}
$$

when $\lambda$ is a partition (i.e. $\left.\lambda_{n} \geq 0\right), s_{\lambda}\left(X_{n}\right)$ is the Schur function, see e.g. [52, Chapter I, $\S 3$.

$2^{0}$ Let $\lambda=\left(\lambda_{1} \geq \lambda_{2} \geq \cdots \geq \lambda_{n} \geq 0\right)$ be a partition, then

$$
\pi_{n}\left(x^{\lambda} \prod_{1 \leq i<j \leq n}\left(1-q x_{j} / x_{i}\right)\right)=v_{\lambda}(q) P_{\lambda}\left(X_{n} ; q\right)
$$

where $P_{\lambda}\left(X_{n} ; q\right)$ denotes the Hall-Littlewood polynomial, see e.g. [52], Chapter III, §1, and

$$
(1-q)^{n} v_{\lambda}(q)=\prod_{i \geq 0}(q ; q)_{\lambda_{i}^{\prime}-\lambda_{i+1}^{\prime}} .
$$


Here $\lambda^{\prime}=\left(\lambda_{1}^{\prime}, \cdots, \lambda_{m}^{\prime}\right)$ is the conjugate partition to $\lambda$; by definition we set $\lambda_{0}^{\prime}=n$, and

$$
(q ; q)_{m}=\prod_{j=1}^{m}\left(1-q^{j}\right) .
$$

Let $\eta=\left(\eta_{1}, \ldots, \eta_{p}\right)$ be a composition of $n$. Denote by $\Phi(\eta)$ the set of ordered pairs $(i, j) \in \mathbb{Z}^{2}$ such that

$$
1 \leq i \leq \eta_{1}+\cdots+\eta_{r}<j \leq n
$$

for some $r, 1 \leq r \leq p$. For example, if $\eta=\left(1^{n}\right)$, then

$$
\Phi(\eta)=\left\{(i, j) \in \mathbb{Z}^{2} \mid 1 \leq i<j \leq n\right\} .
$$

Let $\mu$ be a partition, $l(\mu) \leq n$, and $\eta$ be a composition, $|\eta|=n$. Consider the formal power series $B_{\eta}\left(X_{n} ; q\right), H_{\mu \eta}\left(X_{n} ; q\right)$ and $K_{\lambda \mu \eta}(q)$ defined by

$$
\begin{aligned}
B_{\eta}\left(X_{n} ; q\right) & =\prod_{(i, j) \in \Phi(\eta)}\left(1-q x_{i} / x_{j}\right)^{-1}, \\
H_{\mu \eta}\left(X_{n} ; q\right) & =\pi_{n}\left(x^{\mu} B_{\eta}\left(X_{n} ; q\right)\right) \\
& =\sum_{\lambda} K_{\lambda \mu \eta}(q) s_{\lambda}\left(X_{n}\right),
\end{aligned}
$$

where the sum runs over the set of dominant weights $\lambda=\left(\lambda_{1} \geq \cdots \geq \lambda_{n}\right)$ in $\mathbb{Z}^{n}$.

It is known [62] that the coefficients $K_{\lambda \mu \eta}(q)$ are in fact polynomials in $q$ with integer coefficients. If partitions $\lambda$ and $\mu$, and a composition $\eta$ such that $l(\mu) \leq|\eta|$ are given, we will call the polynomial $K_{\lambda \mu \eta}(q)$ by parabolic Kostka polynomial of type $(\lambda \mu \eta)$, or simply by parabolic Kostka polynomial if no confusion may happen. Note, that by the same formulae (3.3) - (3.5) the polynomials $K_{\lambda \mu \eta}(q)$ may be defined also in the case when $\mu$ is a composition. However, in this general case polynomials $K_{\lambda \mu \eta}(q)$ may have negative coefficients, and their combinatorial and representation theoretical meanings are unclear.

Conjecture 3.2 (Kirillov-Shimozono 40|) Let $\lambda$, $\mu$ be partitions, $|\lambda|=|\mu|, l(\mu)=n$, and $\eta$ be a composition of $n$, then the coefficients $K_{\lambda \mu \eta}(q)$ are polynomials in $q$ with non-negative integer coefficients.

The partition $\mu$ and composition $\eta, l(\mu) \leq|\eta|, l(\eta)=p$, define a sequence of partitions $R=\left(\mu^{(1)}, \ldots, \mu^{(p)}\right), l\left(\mu^{(i)}\right)=\eta_{i}$, by the following rule

$$
\mu^{(i)}=\left(\mu_{\eta_{1}+\cdots+\eta_{i-1}+1}, \cdots, \mu_{\eta_{1}+\cdots+\eta_{i}}\right), \quad 1 \leq i \leq p,
$$

where by definition we put $\eta_{0}:=0$.

Theorem 3.3 (62) Let $\lambda, \mu, \eta$ be as above. Then

$$
K_{\lambda \mu \eta}(1)=\operatorname{Mult}\left[V_{\lambda}: \otimes_{i=1}^{p} V_{\mu^{(i)}}\right]
$$

i.e. $K_{\lambda \mu \eta}(1)$ is equal to the multiplicity of irreducible highest weight $\lambda \mathfrak{g l}(n)-$ module $V_{\lambda}$ in the tensor product of irreducible highest weight $\mu^{(i)}$ representations $V_{\mu^{(i)}}, 1 \leq i \leq p$, of the Lie algebra $\mathfrak{g} l(n)$. 
In the case when all partitions $\mu^{(i)}$ have rectangular shapes, Theorem 3.3 has been proved in 29].

Example 3.4 If $\eta=\left(\eta_{1}, \eta_{2}\right) \in \mathbb{Z}_{\geq 0}^{2}, \eta_{1}+\eta_{2}=n$, and $\mu$ is a partition, $l(\mu) \leq n$, then

$$
K_{\lambda \mu \eta}(q)=q^{c(\lambda, \mu, \eta)} \operatorname{Mult}\left[V_{\lambda}: V_{\mu^{(1)}} \otimes V_{\mu^{(2)}}\right],
$$

for some constant $c(\lambda, \mu, \eta) \in \mathbb{Z}_{\geq 0}$, where $\mu^{(1)}=\left(\mu_{1}, \ldots, \mu_{\eta_{1}}\right), \mu^{(2)}=\left(\mu_{\eta_{1}+1}, \ldots, \mu_{n}\right)$.

Remark 3.5 According to (3.6) and Conjecture 3.2, the parabolic Kostka polynomials $K_{\lambda \mu \eta}(q)$ may be considered as a $q$-analog of the tensor product multiplicities. Another $q$-analog of the tensor product multiplicities had been introduced by Lascoux, Leclerc and Thibon [43]. Formula (3.7) shows that in general these two $q$-analogs are different. However, it was conjectured in [40], Conjecture 5, and [34], Conjecture 6.5, that, in fact, these two $q$-analogs coincide in the case when partition $\mu$ and composition $\eta$ correspond to a dominant sequence of rectangular shape partitions.

In the sequel, we are mainly interested in the case when for a given partition $\mu$ a composition $\eta$ is chosen in such a way that the corresponding sequence of partitions $R=$ $\left(\mu^{(1)}, \ldots, \mu^{(p)}\right)$ contains only rectangular shape partitions, i.e. $\mu^{(a)}=\left(\mu_{a}^{\eta_{a}}\right)$ for all $1 \leq a \leq p$. Conversely, any sequence $R=\left(R_{1}, \ldots, R_{p}\right)$ of rectangular shape partitions $R_{a}=\left(\mu_{a}^{\eta_{a}}\right)$, $1 \leq a \leq p$, such that $\mu_{1} \geq \cdots \geq \mu_{p}$ (we will call such sequence dominant sequence of rectangular shape partitions) defines the partition $\mu$ and composition $\eta$. Namely, the parts of composition $\eta:=\eta(R)=\left(\eta_{1}, \ldots, \eta_{p}\right)$ are equal to the lengths $\eta_{a}:=l\left(R_{a}\right)$ of partitions $R_{a}$. The partition $\mu:=\mu(R)$ is obtained by concatenating the parts of partitions $R_{a}$ in order.

Example 3.6 Take $\lambda=(5,4,4,2,1)$ and $R=((3,3),(2,2,2),(2),(1,1))$. Then $\eta(R)=$ $(2,3,1,2)$ and $\mu(R)=(3,3,2,2,2,2,1,1)$. In this case

$$
K_{\lambda \mu \eta}(q)=q^{5}\left(1+3 q+4 q^{2}+2 q^{3}\right) .
$$

Definition 3.7 The parabolic Kostka polynomial $K_{\lambda R}(q)$ corresponding to a partition $\lambda$ and a dominant sequence of rectangular shape partitions $R$ is defined by the following formula

$$
K_{\lambda R}(q)=K_{\lambda \mu(R) \eta(R)}(q)
$$

We will call the value of parabolic Kostka polynomial $K_{\lambda R}(q)$ at $q=1$ by parabolic Kostka number and denote it by

$$
K_{\lambda R}:=K_{\lambda R}(1)
$$

Theorem 3.8 (41]) Assume that $\lambda$ is a partition and $R$ is a dominant sequence of rectangular shape partitions. Then the parabolic Kostka polynomial $K_{\lambda R}(q)$ has non-negative integer coefficients. 
Examples 3.9 Let $\lambda$ be a partition.

$1^{0}$ Let $R_{a}$ be the single row $\left(\mu_{a}\right)$ for all $a$, where $\mu=\left(\mu_{1}, \mu_{2}, \ldots\right)$ is a partition of length at most $n$. Then

$$
K_{\lambda R}(q)=K_{\lambda \mu}(q),
$$

i.e. $K_{\lambda R}(q)$ coincides with the Kostka-Foulkes polynomial $K_{\lambda \mu}(q)$.

Proof of (3.9) follows from the following, probably well-known, identity

$$
\pi_{n}\left(x^{\mu} \prod_{1 \leq i<j \leq n}\left(1-q x_{i} / x_{j}\right)^{-1}\right)=\sum_{k \geq 0} e_{n}\left(X_{n}\right)^{-k} Q_{\mu+\left(k^{n}\right)}^{\prime}\left(X_{n} ; q\right),
$$

where $e_{n}\left(X_{n}\right)=x_{1} \cdots x_{n} ; Q_{\nu}^{\prime}\left(X_{n} ; q\right)$ denotes the modified Hall-Littlewood polynomial corresponding to partition $\nu$, see e.g. [34]. Recall that

$$
Q_{\nu}^{\prime}\left(X_{n} ; q\right):=Q_{\nu}\left[\frac{X_{n}}{1-q} ; q\right]=\sum_{\mu} K_{\nu \mu}(q) s_{\mu}\left(X_{n}\right) .
$$

See Remark 7.20 for yet another proof of the equality (3.9).

$2^{0}$ Let $R_{a}$ be the single column $\left(1^{\eta_{a}}\right)$ for all $a$, and $\eta=\left(\eta_{1}, \eta_{2}, \ldots\right)$. Then

$$
K_{\lambda R}(q)=\bar{K}_{\lambda^{\prime} \eta^{+}}(q),
$$

the cocharge Kostka-Foulkes polynomial, where $\lambda^{\prime}$ is the conjugate of the partition $\lambda$, and $\eta^{+}$is the partition obtained by sorting the parts of $\eta$ into weakly decreasing order. Formula (3.11) follows from that (3.10) and the duality theorem for parabolic Kostka polynomials.

Duality Theorem $([35,40])$ Let $\lambda$ be a partition, and $R$ be a dominant sequence of rectangular shape partitions, $R=\left(\left(\mu_{a}^{\eta_{a}}\right)\right)_{a=1}^{p}$. Denote by $R^{\prime}$ a dominant rearrangement of the sequence of rectangular shape partitions $\left(\left(\eta_{a}^{\mu_{a}}\right)\right)_{a=1}^{p}$ obtained by transposing each of the rectangular in R. Then

$$
K_{\lambda^{\prime} R^{\prime}}(q)=q^{n(R)} K_{\lambda R}\left(q^{-1}\right),
$$

where $n(R)=\sum_{1 \leq a<b \leq p} \min \left(\mu_{a}, \mu_{b}\right) \min \left(\eta_{a}, \eta_{b}\right)$.

Note that the left hand side of (3.12) is computed in $\mathfrak{g} l(m)$, where $m=\sum \mu_{a}$ is the total number of columns in the rectangles of $R$, whereas the right hand side of (3.12) is computed in $\mathfrak{g l}(n)$, where $n=\sum \eta_{a}$ is the total number of rows in the rectangles of $R$.

$3^{0}$ Let $k$ be a positive integer and $R_{a}$ be the rectangular with $k$ columns and $\eta_{a}$ rows, $|\eta|=n$. Then [70, 62] $K_{\lambda R}(q)$ is the Poincare polynomial of the isotopic component of the irreducible $G L(n)$-module of highest weight $\left(\lambda_{1}-k, \lambda_{2}-k, \ldots, \lambda_{n}-k\right)$ in the coordinate ring of the Zariski closure of the nilpotent conjugacy class whose Jordan form has diagonal block sizes given by the transpose of the partition $\eta^{+}$. In the case $\eta=\left(1^{n}\right)$, the polynomial

$$
K_{\lambda R}(q)=\sum_{\left\{e_{i}\right\}} q^{e_{i}}
$$

gives the Kostant generalized exponents $\left\{e_{i}\right\}$ of representation $V_{\lambda}$. 


\section{Exercises}

1. Let $\eta=\left(\eta_{1}, \ldots, \eta_{n}\right)$ be a composition, $|\eta|=n$. Show that

$$
\pi_{n}\left(\prod_{(i, j) \in \Phi(\eta)}\left(1-q x_{j} / x_{i}\right)\right)=\left[\begin{array}{c}
n \\
\eta_{1}, \ldots, \eta_{n}
\end{array}\right]_{q}
$$

where

$$
\left[\begin{array}{c}
n \\
\eta_{1}, \ldots, \eta_{n}
\end{array}\right]_{q}:=\frac{(q ; q)_{n}}{\prod_{i}(q ; q)_{\eta_{i}}}
$$

denotes the $q$-multinomial coefficient.

2. Take partitions $\lambda=(n+k, n, n-1, \ldots, 3,2)$ and $\mu=\lambda^{\prime}=\left(n, n, n-1, \ldots, 2,1^{k}\right)$. Show that if $n \geq k$, then

$$
K_{\lambda \mu}(q)=q^{2 k-1}\left(\left(\begin{array}{c}
n \\
k-1
\end{array}\right)+q R(q)\right)
$$

for some polynomial $R(q) \in \mathbb{N}[q]$. See also Section 7, Exercise 3e for a generalization of this result.

3. i) Let $\lambda, \mu, \nu$ be partitions, $|\nu|=|\lambda|+|\mu|, l(\lambda)=p, l(\mu) \leq s$. Consider partition

$$
\widetilde{\lambda}=\left(\lambda_{1}+\mu_{1}, \ldots, \lambda_{1}+\mu_{s}, \lambda_{1}, \lambda_{2}, \ldots, \lambda_{p}\right)
$$

and a dominant rearrangement $\widetilde{R}$ of the sequence of rectangular shape partitions $R=\{\nu \cup$ $\left.\left(\lambda_{1}^{s}\right)\right\}$.

- Show that

$$
K_{\widetilde{\lambda}, \widetilde{R}}(q)=q^{Q_{\lambda_{1}}(\nu)-|\lambda|}\left\{c_{\lambda \mu}^{\nu}+\cdots+q^{n(\nu)-n(\lambda)-n(\mu)}\right\},
$$

where $c_{\lambda \mu}^{\nu}$ denotes the Littlewood-Richardson number, i.e.

$$
c_{\lambda \mu}^{\nu}=\operatorname{Mult}\left[V_{\nu}: V_{\lambda} \otimes V_{\mu}\right], \text { and } Q_{\lambda_{1}}(\nu)=\sum_{j \leq \lambda_{1}} \nu_{j}^{\prime}
$$

More generally, let $\lambda \supset \mu$ be partitions such that the complement $\lambda \backslash \mu$ is a disjoint union of partitions $\lambda^{(1)}, \ldots, \lambda^{(p)}$, and $l(\mu)=m$. Let $\nu$ be a partition, define composition $\widetilde{\nu}=(\mu, \nu)$ and partition $\eta=\left(m, 1^{|\nu|}\right)$.

- Show that

$$
K_{\lambda \widetilde{\nu} \eta}(q)=q^{c(\lambda, \mu, \nu)}\left(c_{\lambda^{(1)}, \ldots, \lambda(p)}^{\nu}+\cdots+q^{n(\nu)-n\left(\lambda^{(1)}\right)-\cdots-n\left(\lambda^{(p)}\right)}\right)
$$

where

$$
c_{\lambda^{(1)}, \ldots, \lambda^{(p)}}^{\nu}:=\operatorname{Mult}\left[V_{\nu}: V_{\lambda^{(1)}} \otimes \cdots \otimes V_{\lambda^{(p)}}\right]
$$

denotes the (multiple) Littlewood-Richardson coefficient, and $c(\lambda, \mu, \nu) \in \mathbb{Z}_{\geq 0}$.

It seems plausible that polynomial $K_{\lambda \widetilde{\nu} \eta}(q)$ has non-negative integer coefficients (see Conjecture 3.10), and the degree $d(\lambda, \mu, \nu)$ of parabolic Kostka polynomial $K_{\lambda \widetilde{\nu} \eta}(q)$ is linear, i.e. 
$d(n \lambda, n \mu, n \nu)=n d(\lambda, \mu, \nu)$ for any positive integer $n$ (generalized saturation conjecture for multiple Littlewood-Richardson numbers).

Note also, that the order of parts in the definition of composition $\widetilde{\nu}$ is very essential.

See also Exercise 4 to Section 7 , where connections between this Exercise and the so-called saturation conjecture are explained.

ii) (Ribbon tableaux and parabolic Kostka polynomials)

Let $\lambda \supset \mu$ be partitions such that the complement $\lambda \backslash \mu$ is a disjoint union of partitions $\lambda^{(1)}, \ldots, \lambda^{(p)}$, and $l(\mu)=m$. It is well-known and goes back to D.E. Littlewood (Proc. R. Soc. A 209 (1951), 333-353) that there exists a unique partition $\Lambda$ such that

- $p$-core $(\Lambda)=\emptyset$;

- $p$-quotient $(\Lambda)=\left(\lambda^{(1)}, \ldots, \lambda^{(p)}\right)$, see e.g. [52, Chapter I, Example 8.

It follows from the very definition that

$$
|\Lambda|=p\left(\left|\lambda^{(1)}\right|+\cdots+\left|\lambda^{(p)}\right|\right) .
$$

Now let $\nu$ be a partition such that

$$
|\nu|=\left|\lambda^{(1)}\right|+\cdots+\left|\lambda^{(p)}\right|,
$$

and $\operatorname{Tab}^{(p)}(\Lambda, \nu)$ denotes the set of semistandard $p$-ribbon tableaux of shape $\Lambda$ and weight $\nu$, see e.g. 443, Section 4, or [52, Chapter I, Section 8, Example 8.

Finally, define composition $\widetilde{\nu}=(\mu, \nu)$ and partition $\eta=\left(m, 1^{|\nu|}\right)$, and consider the set $L R(\lambda, \widetilde{\nu}, \eta)$ of Littlewood-Richardson's tableaux of shape $\lambda$ and type $(\widetilde{\nu}, \eta)$, see e.g. 441, Section 2.

- Based on the Stanton-White method (D. Stanton and D. White, J. Comb. Theory A 40 (1985), 211-247) construct a bijection

$$
\pi: \operatorname{Tab}^{(p)}(\Lambda, \nu) \rightarrow L R(\lambda, \widetilde{\nu}, \eta) .
$$

In particular,

$$
K_{\lambda \widetilde{\nu} \eta}(1)=\left|\operatorname{Tab}^{(p)}(\Lambda, \nu)\right|=\epsilon_{p}(\Lambda) K_{\Lambda,} \underbrace{\nu \oplus \cdots \oplus \nu}_{p}\left(\zeta_{p}\right),
$$

where $\zeta_{p}=\exp (2 \pi i / p)$ and $\epsilon_{p}(\Lambda)= \pm 1$. The second equality is due to A. Lascoux, B. Leclerc and J.-Y. Thibon (C.R. Acad. Sci. Paris 316 (1993), 1-6).

Now follow [43], Section 5, denote by $\operatorname{spin}(T)$ the spin of a ribbon tableau $T$. Recall that the spin of a ribbon tableau $T$ is by definition the sum of the spins of its ribbons; the spin of a ribbon $R$ is by definition $(h(R)-1) / 2$, where $h(R)$ denotes the height of $R$; the height of a ribbon is defined to be one less than the number of rows it occupies.

Finally, define the $(q, t)$-ribbon polynomial

$$
c_{\lambda^{(1)}, \ldots, \lambda(p)}^{\nu}(q, t)=\sum_{T \in \operatorname{Tab}^{(p)}(\Lambda, \nu)} t^{\operatorname{spin}(T)} q^{\widetilde{c}(\pi(T))},
$$

where

$$
\widetilde{c}(\pi(T)):=c(\pi(T))-d(\lambda, \mu, \nu) .
$$


From the very definition polynomials $c_{\lambda^{(1)}, \ldots, \lambda^{(p)}}^{\nu}(q, t)$ have the following properties

- $c_{\lambda(1), \ldots, \lambda(p)}^{\nu}(q, t) \in \mathbb{N}[q, t]$

- $c_{\lambda^{(1)}, \ldots, \lambda^{(p)}}^{\nu}(1,1)=\left|\operatorname{Tab}^{(p)}(\Lambda, \nu)\right|$;

Conjecture 3.10 Bijection $\pi$ may be constructed in such a way that

- $c_{\lambda(1), \ldots, \lambda(p)}^{\nu}(q, 1)=K_{\lambda \widetilde{\nu} \eta}(q)$;

- $c_{\lambda(1), \ldots, \lambda^{(p)}}^{\nu}(0, t)=c_{\lambda^{(1)}, \ldots, \lambda^{(p)}}^{\nu}(t)$,

where $c_{\lambda^{(1)}, \ldots, \lambda^{(p)}}^{\nu}(t)$ denotes the $t$-deformation of multiple Littlewood-Richardson numbers introduced by A. Lascoux, B. Leclerc and J.-Y. Thibon (see e.g., B. Leclerc and J.-Y. Thibon, Littlewood-Richardson coefficients and Kazhdan-Lusztig polynomials, math.QA/9809122, 52p.).

4. a. Let $n \geq 1$ be an integer, consider staircase partition

$$
\delta_{n}=(n, n-1, \ldots, 2,1)
$$

and partitions $\mu_{n}=\left(\delta_{n-1}, 1^{n}\right)$, and $\eta_{n}=\left(n-1,1^{n}\right)$. Show that

$$
K_{\delta_{n}, \mu_{n}, \eta_{n}}(q)=q^{n-1} \prod_{j=1}^{n} \frac{1-q^{j}}{1-q} .
$$

b. Let $n \geq 2$ be an integer.

i) Consider staircase partition $\delta_{n}=(n, n-1, \ldots, 2,1)$ and partitions $\mu_{n}=\left(\delta_{n-2}, 1^{2 n-1}\right)$, and $\eta_{n}=\left(n-2,1^{2 n-1}\right)$. Show that

$$
K_{\delta_{n}, \mu_{n}, \eta_{n}}(1)=\frac{2^{2 n}\left(2^{2 n}-1\right)}{2 n}\left|B_{2 n}\right| .
$$

Equivalently,

$$
\sum_{n \geq 1} K_{\delta_{n}, \mu_{n}, \eta_{n}}(1) \frac{x^{2 n-1}}{(2 n-1) !}=\tan x .
$$

Hence, the number of standard Young tableaux of skew shape $\delta_{n} \backslash \delta_{n-2}$ is equal to the tangent number

$$
T_{n}:=\frac{2^{2 n}\left(2^{2 n}-1\right)}{2 n}\left|B_{2 n}\right| .
$$

Here and further $B_{n}$ denotes the $n$-th Bernoulli number. Recall, that

$$
\sum_{n \geq 0} B_{n} \frac{x^{n}}{n !}=\frac{x}{\exp x-1} .
$$

ii) Show that the Kostka-Foulkes polynomial $K_{\delta_{n}, \mu_{n}, \eta_{n}}(q)$ is divisible by the product $\prod_{j=1}^{n-1}\left(1+q^{j}\right)$, and the ratio is a polynomial with non-negative integer coefficients. 
iii) Show that the number of semistandard Young tableaux of skew shape $\delta_{n} \backslash \delta_{n-2}$ and weight $\left(2^{n-1}, 1\right)$ is equal to $2^{-(n-1)} T_{n}$.

iv) Show that the number of semistandard Young tableaux of weight $\left(2^{n-1}\right)$ and skew shape $\delta_{n} \backslash(n-1, n-3, \ldots, 2,1)$ is equal to $2^{n-2}\left|G_{2 n-2}\right|$, where $G_{2 n}$ denotes the $n$-th Genocci number. Recall that

$$
\sum_{n=1}^{\infty} G_{n} \frac{x^{n}}{n !}=\frac{2 x}{1+\exp x}=x\left(1-\tanh \frac{x}{2}\right) .
$$

It is easy to see that

$$
\left|G_{2 n}\right|=2\left(2^{2 n}-1\right)\left|B_{2 n}\right|, \quad G_{2 n+1}=0, \text { if } n>0 .
$$

v) Consider partitions $\alpha_{n}=(n, n-1, \ldots, 3,2), \beta_{n}=\left(\delta_{n-2}, 1^{2 n-2}\right)$, and $\gamma_{n}=\left(n-2,1^{2 n-2}\right)$. Show that

$$
K_{\alpha_{n}, \beta_{n}, \gamma_{n}}(1)=\left|E_{2 n-2}\right|
$$

Equivalently,

$$
\sum_{n \geq 1} K_{\alpha_{n}, \beta_{n}, \gamma_{n}}(1) \frac{x^{2 n-2}}{(2 n-2) !}=\sec x .
$$

Hence, the number of standard Young tableaux of skew shape $(n, n-1, \ldots, 3,2) \backslash \delta_{n-2}$ is equal to the secant (or Euler) number $\left|E_{2 n-2}\right|$.

Here $E_{2 n}$ denotes the $n$-th Euler number. Recall that

$$
\sum_{n \geq 0} E_{2 n} \frac{x^{2 n}}{(2 n) !}=\sec x
$$

Formulae for the numbers $K_{\delta_{n}, \mu_{n}, \eta_{n}}(1)$ from the items $i$ ) and $v$ ) of Exercise $\mathbf{4 b}$ are wellknown, see e.g. H.O. Foulkes (Discr. Math. 15 (1976), 235-252), or I. Gessel and G. Viennot (Adv. Math. 58 (1985), 300-321).

c. i) Denote by $\mathcal{A}_{n}$ the set of alternating (or zigzag) permutations of $n$ letters, i.e.

$$
\mathcal{A}_{n}=\left\{\left(a_{1}, \ldots, a_{n}\right) \in S_{n} \mid \begin{array}{ll}
a_{2 i-1}<a_{2 i}, & i=1, \ldots,\left[\frac{n}{2}\right] \\
a_{2 i}>a_{2 i+1}, & i=1, \ldots,\left[\frac{n-1}{2}\right] .
\end{array}\right\}
$$

Show that

$$
\sum_{n \geq 0}\left|\mathcal{A}_{n}\right| \frac{x^{n}}{n !}=\tan x+\sec x .
$$

This result is due to D. André (J. Math. Pures Appl. 7 (1881), 167-184).

ii) Denote by $\mathcal{S}_{n}$ the set of permutations $w=w_{1} w_{2} \cdots w_{n} \in S_{n}$ satisfying the following conditions

- if $n=2 k$, then $i$ and $i+1$ must precede $i+k$ for $1 \leq i \leq k-1$, while $k$ must precede $2 k$;

- if $n=2 k+1$, then $i$ and $i+1$ must precede $i+k+1$ for $1 \leq i \leq k$. 
Show that

$$
\sum_{n \geq 1}\left|\mathcal{S}_{n}\right| \frac{x^{n}}{n !}=\tan x+\sec x .
$$

This result is due to R. Stanley (Ann. Discr. Math. 6 (1980), 333-342).

iii) Construct bijection between the set of alternating permutations $\mathcal{A}_{n}$ and that of $\mathcal{S}_{n}$.

d. Let $\mathcal{P}_{n}$ be a convex integral polytope in $\mathbb{R}^{n}$ determined by the following inequalities

- $x_{i} \geq 0,1 \leq i \leq n$,

- $x_{i}+x_{i+1} \leq 1,1 \leq i \leq n-1$.

i) Show that the number of vertices of the polytope $\mathcal{P}_{n}$ is equal to the $(n+1)$-th Fibonacci number $F_{n+1}$.

Recall that Fibonacci's numbers $F_{k}$ may be defined using the generating function

$$
\sum_{k \geq 0} F_{k} t^{k}=\frac{1}{1-t-t^{2}}
$$

Question. What are the numbers $f_{i}\left(\mathcal{P}_{n}\right), 0 \leq i \leq n-1$, of $i$-dimensional faces of the polytope $\mathcal{P}_{n}$ ?

ii) Let $\psi_{n}$ denotes the volume of the polytope $\mathcal{P}_{n}$. Show that

$$
\sum_{n \geq 0} \psi_{n} x^{n}=\tan x+\sec x
$$

This Exercise was proposed by R. Stanley (Amer. Math. Monthly 85 (1978), p.197, Problem E2701). For the solution to this problem given by I.G. Macdonald and R.B. Nelsen (independently), see Amer. Math. Monthly 86 (1979), p.396.

iii) Let

$$
\delta\left(\mathcal{P}_{n}\right)=\left(\delta_{0}^{(n)}, \delta_{1}^{(n)}, \ldots, \delta_{n-1}^{(n)}\right)
$$

denotes the $\delta$-vector of the polytope $\mathcal{P}_{n}$, i.e.

$$
\sum_{k \geq 0} i\left(\mathcal{P}_{n} ; k\right) t^{k}=\left(\sum_{k=0}^{n} \delta_{k}^{(n)} t^{k}\right) /(1-t)^{n+1},
$$

where $i\left(\mathcal{P}_{n} ; k\right)$ denotes the number of points $\alpha \in \mathcal{P}_{n}$ such that $k \alpha \in \mathbb{Z}^{n}$.

- Show that

$$
\begin{aligned}
& \delta_{n}^{(n)}=\delta_{n-1}^{(n)}=0, \quad \delta_{0}^{(n)}=\delta_{n-2}^{(n)}=1, \quad \delta_{1}^{(n)}=\delta_{n-3}^{(n)}=F_{n+1}-n-1, \\
& \delta\left(\mathcal{P}_{n} ; t\right)=\sum_{k=0}^{n} \delta_{k}^{(n)} t^{k}=\sum_{w \in \mathcal{S}_{n}} t^{\operatorname{des}(w)} .
\end{aligned}
$$

- Show that $\delta\left(\mathcal{P}_{n} ; t\right)$ is a symmetric polynomial (in the variable $t$ ).

Conjecture 3.11 The $\delta\left(\mathcal{P}_{n} ; t\right)$ is a unimodal polynomial. 
e. i) Let $q=\exp \left(\frac{2 \pi i}{2 k+3}\right)$ be primitive root of unity. For each integer $m$ between 0 and $k$, let $V_{m}$ denote the $(2 m+1)$-dimensional irreducible representation of the quantum universal enveloping algebra $U_{q}(\operatorname{sl}(2))$. Show that

$$
i\left(\mathcal{P}_{n} ; k\right)=\operatorname{Mult}\left(V_{\left[\frac{k+1}{2}\right]}: V_{\left[\frac{k+1}{2}\right]}^{\widehat{\otimes}(n+2)}\right),
$$

i.e. the number of integer points $i\left(\mathcal{P}_{n} ; k\right)$ of the convex integral polytope $k \mathcal{P}_{n}$ is equal to the multiplicity of the irreducible representation $V_{\left[\frac{k+1}{2}\right]}$ of the quantum universal enveloping algebra $U_{q}(s l(2))$ with

$$
q=\exp \left(\frac{2 \pi i}{2 k+3}\right),
$$

in the $(n+2)$-th restricted tensor power of the representation $V_{\left[\frac{k+1}{2}\right]}$. We refer the reader to [27, Chapter 13, Exercise 13.34, for definition and basic properties of the restricted tensor products.

ii) Denote by $A_{k}=\left(a_{i j}\right)$ the $(k+1)$ by $(k+1)$ integer matrix with the following matrix elements:

$$
a_{i j}= \begin{cases}1, & \text { if } i+j \geq k+2 \\ 0, & \text { if } i+j<k+2\end{cases}
$$

- Show that

$$
i\left(\mathcal{P}_{n} ; k\right)=\left(A_{k}^{n+1}\right)_{k+1, k+1},
$$

i.e. the number of integer points of the convex integral polytope $k \mathcal{P}_{n}$ is equal to the $(k+$ $1, k+1)$-entry of the $(n+1)$-th power of the matrix $A_{k}$.

- Show that

$$
A_{k}^{2}=(\min (i, j))_{1 \leq i, j \leq k+1},
$$

and the characteristic polynomial

$$
D_{k}(t):=\operatorname{det}\left(t I_{k+1}-A_{k}^{2}\right),
$$

where $I_{k+1}$ is the identity matrix of order $k+1$, satisfies the recurrence relation

$$
\begin{aligned}
& D_{k}(t)=(1-2 t) D_{k-1}(t)-t^{2} D_{k-2}(t), \quad \text { if } \quad k \geq 2, \\
& D_{0}(t)=1-t, \quad D_{1}(t)=1-3 t+t^{2} .
\end{aligned}
$$

- Show that the roots $t_{a}$ of the characteristic polynomial $D_{k}(t)$ are given by

$$
t_{a}=\frac{1}{4} \sec ^{2} \frac{a \pi}{2 k+3}, \quad 1 \leq a \leq k+1
$$

- Show that

$$
A_{k}^{-2}=\left(b_{i j}\right)_{1 \leq i, j \leq k+1}
$$


where

$$
b_{i j}= \begin{cases}2-\delta_{i, k+1}, & \text { if } i=j \\ -1, & \text { if }|i-j|=1 \\ 0, & \text { if }|i-j| \geq 2\end{cases}
$$

All statements of Exercise e, ii) may be considered as well-known, but we are not able to find precise references where these statements were formulated for the first time.

iii) Let $Z_{n}$ denotes the $n$-element "zigzag poset" with elements $\left\{x_{1}, \ldots, x_{n}\right\}$ and cover relations $x_{2 i-1}<x_{2 i}$ and $x_{2 i}>x_{2 i+1}$.

- Show that

$$
i\left(\mathcal{P}_{n+1} ; k\right)=\Omega\left(Z_{n} ; k\right),
$$

where $\Omega\left(Z_{n} ; k\right)$ denotes the order polynomial of the poset $Z_{n}$. We refer the reader to a book by R. Stanley [66, vol.1, Section 3.11, p.130, for a definition of the order polynomial of a finite poset.

iv) Follow [66], vol.1, Section 3, p.157, consider the generating function

$$
G_{k}(x)=\sum_{n \geq 0} i\left(\mathcal{P}_{n} ; k\right) x^{n}
$$

Show that

$$
G_{1}(x)=1 /(1-x) \text { and } G_{k+1}=\frac{1+G_{k}(x)}{3-x^{2}-G_{k}(x)}, \quad \text { if } k \geq 1 .
$$

This result is due to G. Ziegler, see e.g. [66], vol.1, Chapter 3, Exercise $23 \mathbf{d}$. The generating function $G_{k}(x)$ was computed for the first time by R. Stanley (Ann. Discr. Math. 6 (1980), 333-342, Example 3.2).

Problem 3.12 Compute the two variables generating function

$$
G(x, t)=\sum_{n, k \geq 0} i\left(\mathcal{P}_{n} ; k\right) x^{n} t^{k} .
$$

v) Show that the number of integral points $i\left(\mathcal{P}_{n} ; k\right)$ of the polytope $k \mathcal{P}_{n}$ is equal to the number of $n$-step paths when light ray is reflected from $(k+1)$ glass plates (the so-called multiple reflections or wave sequences).

Further details and references to the Exercise e, v), may be found in

- L. Moser and M. Wyman, The Fibonacci Quarterly 11 (1973), 302-306;

- B. Junge and V. Hoggatt Jr., The Fibonacci Quarterly 11 (1973), 285-291;

- G. Kreweras, Math. Sci. Hum. 14 (1976), n053, 5-30;

- J. Berman and P. Köhler, Mitt. Math. Sem. Giessen 121 (1976), 103-124; and the literature quoted therein.

Remark 3.13 (Fermionic formula for the numbers $i\left(\mathcal{P}_{n} ; k\right)$ ) Consider partitions

$$
\begin{aligned}
\lambda & :=\lambda_{(n, k)}=\left((n+3)\left[\frac{k+1}{2}\right],(n+1)\left[\frac{k+1}{2}\right]\right), \\
\mu & :=\mu_{(n, k)}=(\underbrace{2\left[\frac{k+1}{2}\right], \ldots, 2\left[\frac{k+1}{2}\right]}_{n+2}),
\end{aligned}
$$


and set $l=2 k+1$. It follows from part i) that $i\left(\mathcal{P}_{n} ; k\right)$ is equal to the $l$-restricted Kostka number $K_{\lambda \mu}^{(l)}$.

For the reader's convenience, let us recall [33 a fermionic formula for the $l$-restricted Kostka polynomial $K_{\lambda \mu}^{(l)}(q)$ in the case of two row partition $\lambda=\left(\lambda_{1} \geq \lambda_{2} \geq 0\right)$ and the same size partition $\mu$ :

$$
K_{\lambda \mu}^{(l)}(q)=\sum_{\nu} q^{2 n(\nu)} \prod_{j \geq 1}\left[\begin{array}{c}
P_{j, l}(\nu ; \mu)+m_{j}(\nu) \\
m_{j}(\nu)
\end{array}\right]_{q},
$$

summed over all partitions $\nu=\left(\nu_{1} \geq \nu_{2} \geq \cdots \geq 0\right)$ such that

- $|\nu|=\nu_{1}+\nu_{2}+\cdots=\lambda_{2}, \nu_{1} \leq l$,

- $P_{j, l}(\nu ; \mu):=\sum_{a} \min \left(j, \mu_{a}\right)-\max \left(j+\lambda_{1}-\lambda_{2}-l, 0\right)-2 Q_{j}(\nu) \geq 0$ for all $j \geq 1$.

Here and further we are using the following notation: $Q_{j}(\nu):=\sum_{k \leq j} \nu_{k}^{\prime}, m_{j}(\nu)=\nu_{j}^{\prime}-\nu_{j+1}^{\prime}$ and $n(\nu)=\sum_{i \geq 1}(i-1) \nu_{i}$.

Thus, we come to a fermionic formula,

$$
i\left(\mathcal{P}_{n} ; k\right)=K_{\lambda_{(n, k)}, \mu_{(n, k)}}(1)=\sum_{\nu} \prod_{j \geq 1}\left(\begin{array}{c}
P_{j}(\nu)+m_{j}(\nu) \\
m_{j}(\nu)
\end{array}\right)
$$

summed over all partitions $\nu=\left(\nu_{1} \geq \nu_{2} \geq \cdots \geq 0\right)$ such that

- $|\nu|=\nu_{1}+\nu_{2}+\cdots=(n+1)\left[\frac{k+1}{2}\right], \quad \nu_{1} \leq 2 k+1$,

- $P_{j}(\nu):=(n+2) \min \left(j, 2\left[\frac{k+1}{2}\right]\right)-\max \left(j-2\left[\frac{k}{2}\right]-1,0\right)-2 Q_{j}(\nu) \geq 0$ for all $j \geq 1$.

In particular, if $k=1$ the formula (3.14) gives a "fermionic expression" for Fibonacci's numbers.

vi) Show that

$$
i\left(\mathcal{P}_{n} ; k\right)=\sum_{\nu} \prod_{j \geq 1}\left(\begin{array}{c}
P_{j}(\nu ; n)+m_{j}(\nu) \\
m_{j}(\nu)
\end{array}\right),
$$

summed over all partitions $\nu=\left(\nu_{1}, \nu_{2}, \ldots\right)$ such that

- $\nu_{1} \leq k$;

- $P_{j}(\nu ; n):=(n+1) j-2 Q_{j}(\nu) \geq 0$ for all $j \geq 1$,

where as before $m_{j}(\nu)=\nu_{j}^{\prime}-\nu_{j+1}^{\prime}$, and $Q_{j}(\nu)=\sum_{a} \min \left(j, \nu_{a}\right)$.

More generally, denote by $\mathcal{P}_{n}^{(k)}(r, s)$ the convex polytope

$$
\left\{x=\left(x_{1}, \ldots, x_{n}\right) \in \mathbb{R}_{\geq 0}^{n} \mid x \in k \mathcal{P}_{n}, x_{1} \leq r, x_{n} \leq s\right\} .
$$

- Show that

$$
\#\left|\mathcal{P}_{n}^{(k)}(r, s) \cap \mathbb{Z}^{n}\right|=\sum_{\nu} \prod_{j \geq 1}\left(\begin{array}{c}
P_{j}(\nu ; n, r, s)+m_{j}(\nu) \\
m_{j}(\nu)
\end{array}\right),
$$

summed over all partitions $\nu=\left(\nu_{1}, \nu_{2}, \ldots\right)$ such that

- $\nu_{1} \leq k$; 
- $P_{j}(\nu ; n, r, s):=(n-1) j+\min (j, r)+\min (j, s)-2 Q_{j}(\nu) \geq 0$ for all $j \geq 1$.

Note that the RHS(3.14) and the RHS(3.15) have a different combinatorial structure, but count the same number $\left(=i\left(\mathcal{P}_{n} ; k\right)\right)$. This observation gives rise to an interesting combinatorial identity: $\operatorname{RHS}(3.14)=\operatorname{RHS}(3.15)$. It will be interesting to find a direct combinatorial/bijective proof of the latter.

vii) Consider matrix $M:=M_{k}(x)$ of size $k+1$ by $k+1$ with entries

$$
m_{i j}= \begin{cases}0, & \text { if } i+j<k+2 \\ x^{k-j+1}, & \text { if } i+j \geq k+2\end{cases}
$$

For each integer $r$ between 0 and $k$, define the column vector

$$
|r\rangle=e_{r+1}+\cdots+e_{k+1}=(\underbrace{\overbrace{0, \ldots, 0}^{r}, 1, \ldots, 1}_{k+1})^{t} \in \operatorname{Mat}_{1 \times(k+1)}(\mathbb{Z}) .
$$

Consider the following product of matrices

$$
M\left(q^{N-1} x\right) \ldots M(q x) M(x)|r\rangle:=\left(a_{r, k, N}^{(0)}(x ; q), \ldots, a_{r, k, N}^{(k)}(x ; q)\right)^{t} .
$$

- Show that for each integer $s$ between 0 and $k$, one has

$$
a_{r, k, N}^{(s)}(x ; q)=\sum_{\nu}(q x)^{|\nu|} q^{2 n(\nu)-Q_{r}(\nu)} \prod_{j=1}^{k}\left[\begin{array}{c}
P_{j}(\nu ; r, s)+m_{j}(\nu) \\
m_{j}(\nu)
\end{array}\right]_{q}
$$

summed over all partitions $\nu=\left(\nu_{1}, \nu_{2}, \ldots\right)$ such that

- $\nu_{1} \leq k$;

- $P_{j}(\nu ; r, s):=(N-1) j+\min (j, r)+\min (j, s)-2 Q_{j}(\nu) \geq 0$ for all $j \geq 1$;

- $n(\nu)=\sum(i-1) \nu_{i} ; \quad m_{j}(\nu)=\nu_{j}^{\prime}-\nu_{j+1}^{\prime}, \quad Q_{j}(\nu)=\sum_{a} \min \left(j, \nu_{a}\right)$.

Show that

$$
a_{r, k, N}^{(s)}(x ; q)=\sum_{\left(b_{1}, \ldots, b_{N}\right) \in \mathcal{P}_{N}^{(k)}(r, s)} x^{\sum b_{j}} q^{\sum j b_{j}} .
$$

As far as I am aware, the product of matrices

$$
M_{k}\left(q^{N-1} x\right) \ldots M_{k}(q x) M_{k}(x)|r\rangle
$$

was invented by B. Feigin (private communication, 1992) as a tool for obtaining a fermionic formula for the level $k$ vacuum representation of the affine Lie algebra $\widehat{s l}_{2}$, and that for the characters of certain unitary representations of the Virasoro algebra. This product of matrices was studied further by E. Frenkel and A. Szenes (Int. Math. Res. Notices 2 (1993), 53-60) and by A.N. Kirillov Fusion algebra and Verlinde formula, Preprint of Isaac Newton Institute IN-92019, and hep-th/9212084. To our knowledge, the fermionic formula (3.17) for polynomials $a_{r, k, N}^{(s)}(x ; q)$ was obtained for the first time in 33, p.99. 
f. Consider the sets

$$
\mathcal{S}_{n}^{(i, j)}=\left\{w=w_{1} \cdots w_{n} \in \mathcal{S}_{n} \mid w_{1}=i, w_{n}=j\right\}, \quad \text { and } \mathcal{S}_{n}^{(i)}=\bigcup_{j \in[1, n]} \mathcal{S}_{n}^{(i, j)} .
$$

Show that

i) $\sum_{w \in \mathcal{S}_{n+1}^{(1)}} t^{\operatorname{des}(w)}=\delta\left(\mathcal{P}_{n} ; t\right)=\sum_{u \in \mathcal{S}_{n}} t^{\operatorname{des}(u)}$;

ii) if $n \equiv 0(\bmod 2)$, then

$$
\sum_{w \in S_{n}^{(i, j)}} t^{\operatorname{des}(w)}=\sum_{u \in S_{n}^{(n-j+1, n-i+1)}} t^{\operatorname{des}(u)}, \text { and } S_{n}^{(n)}=\left\{u=w n \mid w \in S_{n-1}\right\} ;
$$

iii) if $n=2 k+1, k \geq 1$, then

$$
\sum_{w \in S_{n}^{(i, j)}} t^{\operatorname{des}(w)}=\sum_{u \in S_{n}^{(k+2-i, 3 k+3-j)}} t^{n-2-\operatorname{des}(u)} .
$$

Let us denote by $\mathcal{S}_{n}^{*}$ the set of all permutations $w=w_{1} \cdots w_{n}$ such that the permutation $\widetilde{w}=1\left(w_{1}+1\right) \cdots\left(w_{n}+1\right)$ belongs to the set $\mathcal{S}_{n+1}$. It follows from part i) that $\left|\mathcal{S}_{n}^{*}\right|=\left|\mathcal{S}_{n}\right|$.

Example 3.14 Consider the case $n=5$. Then

$$
\begin{aligned}
\mathcal{A}_{5}= & \{13254,14253,14352,15243,15342,23154,24153,24351,25143,25341, \\
& 34152,34251,35142,35241,45132,45231\} ; \\
\mathcal{S}_{5}= & \{12345,12354,12435,13245,13254,21345,21435,21354,23145,23154, \\
& 23514,31245,31254,32514,32154,32145\} ; \\
\mathcal{S}_{5}^{*}= & 12345,12435,12354,12453,13245,12543,12534,13254,21354,21345, \\
& 21435,21453,21543,21534,25134,25143\} .
\end{aligned}
$$

Hence, $\delta\left(\mathcal{P}_{5} ; t\right)=1+7 t+7 t^{2}+t^{3}$;

$$
\sum_{w \in \mathcal{S}_{5}} t^{\operatorname{des}(w)} q^{\text {majw }}=1+t\left(2 q+2 q^{2}+2 q^{3}+q^{4}\right)+t^{2}\left(q^{3}+2 q^{4}+2 q^{5}+2 q^{6}\right)+t^{3} q^{7} ;
$$

$\psi_{5}:=\operatorname{vol}\left(\mathcal{P}_{5}\right)=\frac{2^{6}\left(2^{6}-1\right)}{6 !} B_{6}=\frac{2}{15}$.

For the reader's convenience, we list below (for $n=5$ ) some classes of permutations $w \in S_{n}$ which are equinumerous to the set of alternating permutations $\mathcal{A}_{n}$ :

- augmented André permutations of the first kind (see e.g., M. Purtill, Trans. AMS 338 (1993), 77-104, and the literature quoted therein)

$$
\begin{aligned}
& \widetilde{\mathcal{A}}_{5}^{I}=\{12345,12435,13425,23415,13245,14235,34125,24135,23145,21345, \\
&41235,31245,21435,32415,41325,31425\}
\end{aligned}
$$


- André permutations of the second kind (D. Foata and M.-P. Schützenberger, in A survey of Combinatorial Theory, J.N. Srivastava et al. eds., Amsterdam, 1973, 173-187)

$$
\begin{aligned}
\mathcal{A}_{5}^{I I}= & \{12345,12534,14523,34512,15234,14235,34125,45123,35124,51234, \\
& 41235,31245,51423,53412,41523,31524\} ;
\end{aligned}
$$

- augmented simsun permutations (see e.g., R. Stanley, Math. Z. 216 (1994), p.498)

$$
\begin{aligned}
\widetilde{\mathcal{S S}}_{5}= & \{12345,12435,13245,13425,14235,21345,21435,23145,23415,24135, \\
& 31425,31245,34125,41235,41325,42315\}
\end{aligned}
$$

- Jacobi permutations (G. Viennot, Journ. Comb. Th. A 29 (1980), p.124, Definition 3)

$$
\begin{aligned}
J_{5}= & \{15432,35421,52431,42531,32541,54132,53142,13542,43152,52143, \\
& 15243,42153,14253,32154,13254,15432\} .
\end{aligned}
$$

It looks very challenging to construct bijections between the sets $\mathcal{A}_{n}, \widetilde{\mathcal{A}}_{n}^{I}, \mathcal{A}_{n}^{I I}, \mathcal{S}_{n}, \mathcal{S}_{n}^{*}$, $\widetilde{\mathcal{S S}}_{n}$ and $J_{n}$.

g. Let $n \geq 2$, denotes by $\widetilde{\mathcal{P}}_{n}$ the convex (rational) polytope in $\mathbb{R}^{n}$ determined by the following inequalities

- $x_{i} \geq 0,1 \leq i \leq n$,

- $x_{i}+x_{i+1} \leq 1,1 \leq i \leq n-1$,

- $x_{1}+x_{n} \leq 1$.

Show that

$$
\sum_{n \geq 1} \operatorname{vol}\left(\widetilde{\mathcal{P}}_{n}\right) x^{n-1}=\frac{1}{2}(\tan x+\sec x)
$$

i.e.

- $\operatorname{vol}\left(\mathcal{P}_{2 k-1}\right)=2 \operatorname{vol}\left(\widetilde{\mathcal{P}}_{2 k}\right)$, if $k \geq 1$,

- $\operatorname{vol}\left(\mathcal{P}_{2 k}\right)=2 \operatorname{vol}\left(\widetilde{\mathcal{P}}_{2 k+1}\right)$, if $k \geq 0$,

where by definition we set $\widetilde{\mathcal{P}}_{1}:=[0,1 / 2]$.

5. Let us fix $n \geq 3$. i) Consider partitions $\lambda_{n}=(n+1, n, \ldots, 4,3), \mu_{n}=(n-2, n-$ $\left.3, \ldots, 2,1^{3 n-3}\right)$, and $\eta_{n}=\left(n-2,1^{3 n-3}\right)$. Show that

$$
K_{\lambda_{n} \mu_{n} \eta_{n}}(1)=\frac{(3 n-3) !\left(2^{2 n-2}-2\right)}{(2 n-2) !}\left|B_{2 n-2}\right|,
$$

and thus, the number in the RHS(3.19) is equal to the number of standard (skew) Young tableaux of shape $(n+1, n, \ldots, 3) \backslash(n-2, n-3, \ldots, 1)$. This result is due to I. Gessel and G. Viennot [16], §11, and A.N. Kirillov [37]. It has been shown in [37 that

$$
(3 g) ! \operatorname{det}\left|\frac{1}{(3-2 i+2 j) !}\right|_{1 \leq i, j \leq g}=\frac{(3 g) !\left(2^{2 g}-2\right)}{(2 g) !}\left|B_{2 g}\right|=(3 g) ! \operatorname{vol}\left(\mathcal{N}_{g+1}\right),
$$


where $\operatorname{vol}\left(\mathcal{N}_{g}\right)$ stands for the volume of the moduli space $\mathcal{N}_{g}$ of stable rank 2 vector bundles over a smooth complex curve of genus $g$.

ii) Consider partitions $\lambda_{n}=(n+1, n, \ldots, 4,3,2,1)$, $\mu_{n}=\left(n-2, n-3, \ldots, 2,1^{3 n}\right)$, and $\eta_{n}=\left(n-2,1^{3 n}\right)$. Show that

$$
K_{\lambda_{n} \mu_{n} \eta_{n}}(1)=2^{2 n} \frac{(3 n) !}{(2 n) !}\left|B_{2 n}\right| .
$$

6. Let $m, n \geq 2$ and $N \geq n$ be integers. Consider partitions $\lambda_{N}=N(n, n-1, \ldots, 2,1)+$ $(m, \underbrace{0, \ldots, 0}_{n-1}), \mu_{N}=N(n, n-1, \ldots, 2,1)+(\underbrace{0, \ldots, 0}_{n}, m)$, and a composition $\eta=(1, n-2,1)$.

Show that if $m \geq n-1$, then

$$
K_{\lambda_{N}, \mu_{N}, \eta}(q)=\sum_{k=0}^{m}\left(\begin{array}{c}
n-2+k \\
k
\end{array}\right) q^{k+m}
$$

7. Let $\alpha$ and $\beta$ be partitions, and $n, s$ be integer numbers such that $s \geq|\beta|$. Define $m:=s-|\beta| \geq 0$, and consider partition

$$
\lambda:=[\alpha, \beta]_{s}=(\alpha_{1}+s, \ldots, \alpha_{p}+s, \underbrace{s, \ldots, s}_{n-p-t}, s-\beta_{t}, \ldots, s-\beta_{1}),
$$

where $p=l(\alpha), t=l(\beta)$, and the dominant sequence of partitions

$$
R:=R_{n}^{r, s}=\{\underbrace{(1), \ldots,(1)}_{r}, \underbrace{\left(1^{n-1}\right), \ldots,\left(1^{n-1}\right)}_{s}\},
$$

where $r:=|\alpha|-|\beta|+s$. Let us introduce notation $S_{m}:=R_{m}^{m, m}$, and assume additionally that $n \geq m+l(\alpha)+l(\beta)$.

i) Show that

$$
K_{\lambda R}(q) \doteq\left[\begin{array}{c}
r \\
m
\end{array}\right]_{q}\left[\begin{array}{c}
s \\
m
\end{array}\right]_{q} K_{\alpha,(1|\alpha|)}(q) K_{\beta,(1|\beta|)}(q) K_{\left(m^{m}\right), S_{m}}(q) .
$$

ii) Show that

$$
K_{\left(m^{m}\right), S_{m}}(1)=m !,
$$

and $K_{\lambda R}(1)$ is equal to the number $c_{r, s}^{[\alpha, \beta]}$ of up-down staircase tableaux of shape

$$
\{\alpha, \beta\}_{n}:=(\alpha_{1}, \ldots, \alpha_{p-1}, \alpha_{p}, \underbrace{0, \ldots, 0}_{n-p-t},-\beta_{t}, \ldots,-\beta_{1})
$$

and type $\epsilon_{r, s}:=(\underbrace{1, \ldots, 1}_{r}, \underbrace{-1, \ldots,-1}_{s})$, see [67], Definition 4.4. In particular, the number $a_{2 s}^{[\alpha, \beta]}$ of alternating (or oscillating) tableaux, i.e. up-down staircase tableaux of shape $\{\alpha, \beta\}_{n}$ and type $\epsilon=(\underbrace{1,-1,1,-1, \ldots, 1,-1}_{2 s}),[i b i d]$, is equal to

$$
(s-|\alpha|) !\left(\begin{array}{c}
s \\
|\alpha|
\end{array}\right)^{2} f^{\alpha} f^{\beta}, \text { if } n \geq s-|\alpha|+l(\alpha)+l(\beta) .
$$


Formulae for the numbers $c_{r, s}^{[\alpha, \beta]}$ and $a_{2 s}^{[\alpha, \beta]}$ are due to J. Stembridge [67], Proposition 4.8, and in some particular cases to P. Hanlon [24]. Formula (3.21) may be considered as a $q$-analog of the above results obtained by J. Stembridge.

Examples 3.15 $1^{0}$ Take $\alpha=\beta=(2,2)$ and $n=s=6$. Then $\lambda:=[\alpha, \beta]_{6}=(8,8,6,6,4,4)$, $m=2$, and $R:=R_{6}^{6,6}=\{\underbrace{(1), \ldots,(1)}_{6}, \underbrace{\left(1^{5}\right), \ldots,\left(1^{5}\right)}_{6}\}$. Using the fermionic formula (4.1), one can check that

$$
K_{\lambda R}(q)=q^{98}\left(1+q^{2}\right)^{3}\left[\begin{array}{l}
6 \\
2
\end{array}\right]_{q}^{2}, \quad K_{\alpha,\left(1^{4}\right)}(q)=q^{2}+q^{4}=K_{\left(2^{2}\right), S_{2}}(q) .
$$

$2^{0}$ Take $\alpha=\beta=(2,1), n=7$, and $r=s=6$. Then $\lambda:=[\alpha, \beta]_{7}=(8,7,6,6,6,5,4), m=3$, and $R:=R_{7}^{6,6}=\{\underbrace{(1), \ldots,(1)}_{6}, \underbrace{\left(1^{6}\right), \ldots,\left(1^{6}\right)}_{6}\}$. Using the fermionic formula (4.1), one can check that

$$
\begin{aligned}
& K_{\lambda R}(q)=q^{95}(1+q)^{2}\left[\begin{array}{l}
6 \\
3
\end{array}\right]_{q}^{2}\left(1+q^{2}+2 q^{3}+q^{4}+q^{6}\right) \\
& K_{(2,1),\left(1^{3}\right)}(q)=q+q^{2} \\
& K_{(333), S_{3}}(q)=q^{9}\left(1+q^{2}+2 q^{3}+q^{4}+q^{6}\right) .
\end{aligned}
$$

iii) Show that if $r \geq|\alpha|, s \geq|\beta|$, and $r-s=|\alpha|-|\beta|$, then

$$
K_{[\alpha, \beta]_{n+1}, R_{n+1}^{r, s}}(q) \geq K_{[\alpha, \beta]_{n}, R_{n}^{r, s}}(q)
$$

for all $n \geq l(\alpha)+l(\beta)$, and if $n \geq s-|\beta|+l(\alpha)+l(\beta)$, then the $\operatorname{LHS}(3.22)$ is equal to the $\operatorname{RHS}(3.22)$.

Example 3.16 Take $\alpha=\beta=(2,1)$, and $r=s=6$. Using the fermionic formula (4.1), one can check that

$$
K_{[\alpha, \beta]_{7}, R_{7}^{6,6}}(q)-K_{[\alpha, \beta]_{6}, R_{6}^{6,6}}(q)=q^{98}\left(1+q+q^{2}+q^{3}+q^{4}\right)^{2} .
$$

Questions. i) Let $\lambda$ be a partition and $R=\left\{R_{a}=\left(\mu_{a}^{\eta_{a}}\right)\right\}_{a=1}^{l}$ be a dominant sequence of rectangular shape partitions. Under what conditions on $\lambda$ and $R$ the parabolic Kostka number $K_{\lambda R} \neq 0$ ?

One obvious necessary condition is the following: $\lambda \geq \mu(R)$ with respect to the dominance ordering on the set of partitions. Let us recall, see Section 3 , that $\mu(R)$ denotes the partition which is obtained by concatenating the parts of partitions $R_{a}$ in order.

ii) When does the parabolic Kostka number $K_{\lambda, R}$ equal to 1 ?

For example,

$$
K_{(8 n, 8 n, 8 n, 8 n, 8 n),((5 n, 5 n),(5 n, 5 n),(5 n, 5 n),(5 n, 5 n))}(q)=q^{24 n} .
$$

iii) Does there exist an explicit formula for computing the degree of parabolic Kostka polynomial $K_{\lambda R}(q)$ ? Recall that $\operatorname{deg} K_{\lambda \mu}(q)=n(\mu)-n(\lambda)$, see e.g. [52], Chapter I. It looks plausible that the degree $d(\lambda, \mu ; \eta)$ of parabolic Kostka polynomial $K_{\lambda \mu \eta}(q)$ is a homogeneous degree 1 function, i.e. $d(n \lambda, n \mu ; \eta)=n d(\lambda, \mu ; \eta)$ for any positive integer $n$. 
Example 3.17 Below we list some "exotic examples" of Kostka-Foulkes and parabolic Kostka polynomials:

$$
\begin{aligned}
K_{(42),\left(2,1^{4}\right)}(q) & =q^{4}(11211), \\
K_{(442),(3,3,2,1,1)}(q) & =q^{2}(11311), \\
K_{(4422),(3,3,(2,2), 1,1)}(q) & =q^{4}(11411), \\
K_{(4431),(3,(2,2),(2,2), 1)}(q) & =2 q^{4}(111), \\
K_{(444222),(3,(3,3),(2,2,2), 2,1)}(q) & =q^{4}(2332), \\
K_{(664),((3,3),(3,3), 2,2)}(q) & =q^{8}(21211) \\
K_{(654321),(4,(3,3,3),(2,2),(1,1,1,1))}(q) & =3 q^{6}(1+q)^{3}, \\
K_{(7654321),((4,4,4),(3,3,3,3), 2,(1,1))}(q) & =3 q^{7}(1+q)^{4}, \\
K_{(7654321),((4,4,4,4), 4,2,(1,1),(1,1,1,1))}(q) & =q^{8}(5,17,24,17,5), \\
K_{(87654321),(5,(4,4,4,4),(3,3,3),(1,1,1,1,1), 1)}(q) & =6 q^{11}(1+q)^{4}\left(2+3 q+2 q^{2}\right), \\
K_{(2 n, 2 n, 2 n, 2 n),((n, n),(n, n),(n, n),(n, n))}(q) & =q^{4 n}\left[\begin{array}{c}
n+2 \\
2
\end{array}\right]
\end{aligned}
$$

More generally, let us take $\lambda=(\underbrace{2 n, \ldots, 2 n}_{2 k})$, and $R=\left(\left(n^{k}\right),\left(n^{k}\right),\left(n^{k}\right),\left(n^{k}\right)\right)$. Then

$$
K_{\lambda, R}(q)=q^{2 k n}\left[\begin{array}{c}
n+k \\
k
\end{array}\right]_{q^{2}} .
$$

Indeed, there are exactly $\left(\begin{array}{c}n+k \\ k\end{array}\right)$ admissible configurations of type $(\lambda ; R)$, each of those has zero essential vacancy numbers only. Similarly, let $n>m \geq 0$ and $k \geq 1$ be integers, consider partition $\lambda=(\underbrace{2 n, \ldots, 2 n}_{2 k}, \underbrace{2 m, \ldots, 2 m}_{2 k})$ and sequence of rectangular shape partitions

$$
R=\left(\left((m+n)^{k}\right),\left((m+n)^{k}\right),\left((m+n)^{k}\right),\left((m+n)^{k}\right)\right) .
$$

Then

$$
\begin{gathered}
K_{\lambda, R}(q)=q^{2 k(n-m)}\left[\begin{array}{c}
k+n-m \\
k
\end{array}\right]_{q^{2}} . \\
K_{(9621),((3,3),(3,3), 3,3)}(q)=q^{13}(25652) \doteq s_{21}\left(1, q, q, q^{2}\right) .
\end{gathered}
$$

See also Section 6, Exercise 14 for a generalization of the latter example.

\section{Fermionic formula for parabolic Kostka polynomials}

Let $\lambda$ be a partition and $R=\left(\left(\mu_{a}^{\eta_{a}}\right)\right)_{a=1}^{p}$ be a sequence of rectangular shape partitions such that

$$
|\lambda|=\sum_{a}\left|R_{a}\right|=\sum_{a} \mu_{a} \eta_{a}
$$


Definition 4.1 The configuration of type $(\lambda ; R)$ is a sequence of partitions $\nu=\left(\nu^{(1)}, \nu^{(2)}, \ldots\right)$ such that

$$
\left|\nu^{(k)}\right|=\sum_{j>k} \lambda_{j}-\sum_{a \geq 1} \mu_{a} \max \left(\eta_{a}-k, 0\right)=-\sum_{j \leq k} \lambda_{j}+\sum_{a \geq 1} \mu_{a} \min \left(k, \eta_{a}\right)
$$

for each $k \geq 1$.

Note that if $k \geq l(\lambda)$ and $k \geq \eta_{a}$ for all $a$, then $\nu^{(k)}$ is empty. In the sequel (except Corollary 4.4) we make the convention that $\nu^{(0)}$ is the empty partition.

For a partition $\mu$ define the number $Q_{n}(\mu)=\mu_{1}^{\prime}+\cdots+\mu_{n}^{\prime}$, which is equal to the number of cells in the first $n$ columns of $\mu$. The vacancy numbers $P_{n}^{(k)}(\nu):=P_{n}^{(k)}(\nu ; R)$ of the configuration $\nu$ of type $(\lambda ; R)$ are defined by

$$
P_{n}^{(k)}(\nu)=Q_{n}\left(\nu^{(k-1)}\right)-2 Q_{n}\left(\nu^{(k)}\right)+Q_{n}\left(\nu^{(k+1)}\right)+\sum_{a \geq 1} \min \left(\mu_{a}, n\right) \delta_{\eta_{a}, k}
$$

for $k, n \geq 1$, where $\delta_{i, j}$ is the Kronecker delta.

Definition 4.2 The configuration $\nu$ of type $(\lambda ; R)$ is called admissible, if

$$
P_{n}^{(k)}(\nu ; R) \geq 0 \text { for all } k, n \geq 1
$$

We denote by $C(\lambda ; R)$ the set of all admissible configurations of type $(\lambda ; R)$, and call the vacancy number $P_{n}^{(k)}(\nu ; R)$ essential, if $m_{n}\left(\nu^{(k)}\right)>0$.

Finally, for configuration $\nu$ of type $(\lambda ; R)$ let us define its charge

$$
c(\nu)=\sum_{k, n \geq 1}\left(\begin{array}{c}
\alpha_{n}^{(k-1)}-\alpha_{n}^{(k)}+\sum_{a} \theta\left(\eta_{a}-k\right) \theta\left(\mu_{a}-n\right) \\
2
\end{array}\right)
$$

and cocharge

$$
\bar{c}(\nu)=\sum_{k, n \geq 1}\left(\begin{array}{c}
\alpha_{n}^{(k-1)}-\alpha_{n}^{(k)} \\
2
\end{array}\right)
$$

where $\alpha_{n}^{(k)}=\left(\nu^{(k)}\right)_{n}^{\prime}$ denotes the size of the $n$-th column of the $k$-th partition $\nu^{(k)}$ of the configuration $\nu$; for any real number $x \in \mathbb{R}$ we put $\theta(x)=1$, if $x \geq 0$, and $\theta(x)=0$, if $x<0$.

Theorem 4.3 (Fermionic formula for parabolic Kostka polynomials [35, 41]) Let $\lambda$ be a partition and $R$ be a dominant sequence of rectangular shape partitions. Then

$$
K_{\lambda R}(q)=\sum_{\nu} q^{c(\nu)} \prod_{k, n \geq 1}\left[\begin{array}{c}
P_{n}^{(k)}(\nu ; R)+m_{n}\left(\nu^{(k)}\right) \\
m_{n}\left(\nu^{(k)}\right)
\end{array}\right]_{q}
$$

summed over all admissible configurations $\nu$ of type $(\lambda ; R) ; m_{n}(\lambda)$ denotes the number of parts of the partition $\lambda$ of size $n$. 
Corollary 4.4 (Fermionic formula for Kostka-Foulkes polynomials [30]) Let $\lambda$ and $\mu$ be partitions of the same size. Then

$$
K_{\lambda \mu}(q)=\sum_{\nu} q^{c(\nu)} \prod_{k, n \geq 1}\left[\begin{array}{c}
P_{n}^{(k)}(\nu, \mu)+m_{n}\left(\nu^{(k)}\right) \\
m_{n}\left(\nu^{(k)}\right)
\end{array}\right]_{q},
$$

summed over all sequences of partitions $\nu=\left\{\nu^{(1)}, \nu^{(2)}, \ldots\right\}$ such that

- $\left|\nu^{(k)}\right|=\sum_{j>k} \lambda_{j}, k=1,2, \ldots$;

- $P_{n}^{(k)}(\nu, \mu):=Q_{n}\left(\nu^{(k-1)}\right)-2 Q_{n}\left(\nu^{(k)}\right)+Q_{n}\left(\nu^{(k+1)}\right) \geq 0$ for all $k, n \geq 1$, where by definition we put $\nu^{(0)}=\mu$;

$$
\text { - } c(\nu)=\sum_{k, n \geq 1}\left(\begin{array}{c}
\left(\nu^{(k-1)}\right)_{n}^{\prime}-\left(\nu^{(k)}\right)_{n}^{\prime} \\
2
\end{array}\right) .
$$

\section{Exercises}

1. Show that

$$
\sum_{\nu} q^{c(\nu)} \prod_{k, n \geq 1}\left[\begin{array}{c}
P_{n}^{(k)}(\nu ; R)+m_{n}\left(\nu^{(k)}\right) \\
m_{n}\left(\nu^{(k)}\right)
\end{array}\right]_{q}^{\prime}=0,
$$

where the sum is taken over the set of all non-admissible configurations of type $(\lambda ; R)$, i.e. configurations $\nu$ of type $(\lambda ; R)$ such that

$$
P_{n}^{(k)}(\nu ; R)+m_{n}\left(\nu^{(k)}\right)<0
$$

for some $k, n \geq 1$. Here, for any integer $N$ and non-negative integer $M$ we use the notation $\left[\begin{array}{l}N \\ M\end{array}\right]_{q}^{\prime}$ to denote the modified $q$-binomial coefficient

$$
\left[\begin{array}{l}
N \\
M
\end{array}\right]_{q}^{\prime}=\frac{\left(1-q^{N}\right)\left(1-q^{N-1}\right) \cdots\left(1-q^{N-M+1}\right)}{(1-q)\left(1-q^{2}\right) \cdots\left(1-q^{M}\right)} .
$$

2. (KOH's identity) Follow [71], denote by $G(n, k)$ the $q$-binomial coefficient $\left[\begin{array}{c}n+k \\ k\end{array}\right]_{q}$ (so that $G(n, k)=0$, if either $n<0$ or $k<0)$.

Show that

$$
G(n, k)=\sum_{\nu \vdash k} q^{2 n(\nu)} \prod_{j=1}^{k} G\left((n+2) j-2 k+\sum_{l \geq j}(l-j) d_{l}, d_{j}\right),
$$

summed over all partitions $\nu=\left(1^{d_{1}}, 2^{d_{2}}, \ldots, k^{d_{k}}\right)$ of $k$;

$$
2 n(\nu)=\sum_{j \geq 1} j d_{j}^{2}+2 \sum_{i<j} \min (i, j) d_{i} d_{j}-k .
$$


Binomial identity (4.5) is due to Zeilberger [71] and may be considered as an algebraic version of the main result, Theorem 2.13, of K. O'Hara's paper [56]. Note that if one replaces in the identity (4.5) the $q$-binomial coefficient $G(n, k)$ by the modified $q$-binomial coefficient $\left[\begin{array}{c}n+k \\ k\end{array}\right]_{q}^{\prime}$, see definition in the previous Exercise 1, then this new version of the identity (4.5) becomes "much more elementary", and a short algebraic proof of it was found by Macdonald [50].

Remark 4.5 Binomial identity (4.5) is a particular case of the identity (1.4) in the case $N=k+n$ and $\lambda=\left(1^{k}\right)$. Note that the symmetry relation

$$
\left[\begin{array}{c}
n+k \\
k
\end{array}\right]=\left[\begin{array}{c}
n+k \\
n
\end{array}\right]
$$

gives rise to a highly nontrivial identity between binomial sums.

Problem 4.6 Let $\lambda$ and $\mu$ be partitions, $l(\mu) \leq n$, and $\eta$ be a composition of $n$. Find a fermionic formula for the parabolic Kostka polynomial $K_{\lambda \mu \eta}(q)$ which generalizes that (4.1).

3. (Domino tableaux and configurations)

i) Let $\lambda$ and $\mu$ be partitions such that $l(\mu)=s$, and $C(\lambda ; \mu)$ be the corresponding set of admissible configurations. Denote by $C_{(2)}(\lambda ; \mu)$ the subset of $C(\lambda ; \mu)$ consisting of all configurations $\nu$ such that

$$
P_{n}^{(k)}(\nu) m_{n}\left(\nu^{(k)}\right) \equiv 0(\bmod 2) \text { for all } n, k \geq 1 \text {. }
$$

Conditions (4.6) may be rewritten in the following form

$$
\left[P_{n}^{(k)}(\nu) / 2\right]+\left[m_{n}\left(\nu^{(k)}\right) / 2\right]=\left[\left(P_{n}^{(k)}(\nu)+m_{n}\left(\nu^{(k)}\right)\right) / 2\right],
$$

where for any $x \in \mathbb{R}$, the symbol $[x]$ stands for the nearest integer which is smaller or equal to $x$. Finally, let $c(\nu)$ denotes the charge of configuration $\nu$, see (4.3) for definition.

- Show that if $\nu \in C_{(2)}(\lambda ; \mu)$, then

$$
c(\nu) \equiv \epsilon(\lambda)(\bmod 2)
$$

where $\epsilon(\lambda)=\lambda_{1}^{\prime}+\lambda_{3}^{\prime}+\lambda_{5}^{\prime}+\cdots$.

Next, for any configuration $\nu \in C_{(2)}(\lambda ; \mu)$ define its spin by

$$
\operatorname{spin}(\nu)=\frac{1}{2} \sum_{k, n \geq 1}\left\{\frac{\left(\nu^{(k-1)}\right)_{n}^{\prime}-\left(\nu^{(k)}\right)_{n}^{\prime}}{2}\right\}
$$

where for any $x \in \mathbb{R}$, the symbol $\{x\}, 0 \leq\{x\}<1$, denotes the fractional part of $x$. In other words, the $\operatorname{spin}(\nu)$ of a configuration $\nu$ is equal to the one fourth of the number of odd 
numbers appearing among the all differences $\left(\nu^{(k-1)}\right)_{n}^{\prime}-\left(\nu^{(k)}\right)_{n}^{\prime}, k, n \geq 1$. Recall that by definition $\nu^{(0)}=\mu$.

Finally, for any partition $\tau$ such that $|\tau|=|\lambda|+|\mu|$ define the $(q, t)$-domino polynomial

$$
c_{\Lambda}^{\tau}(q, t)=\sum_{\nu \in C_{(2)}(\Lambda ; \tau \oplus \tau)} t^{\operatorname{spin}(\nu)} q^{\widetilde{c}(\nu)} \prod_{k, n \geq 1}\left[\begin{array}{c}
\widehat{P}_{n}^{(k)}(\nu)+\widehat{m}_{n}\left(\nu^{(k)}\right) \\
\widehat{m}_{n}\left(\nu^{(k)}\right)
\end{array}\right]_{q},
$$

where

( $\alpha) \Lambda$ is a unique partition such that $2-\operatorname{core}(\Lambda)=\emptyset$ and $2-$ quotient $(\Lambda)=(\lambda, \mu)$;

$(\beta)$ for any configuration $\nu \in C_{(2)}(\Lambda ; \tau \oplus \tau), \widetilde{c}(\nu):=(c(\nu)-\epsilon(\lambda)) / 2$ denotes its normalized charge;

$(\gamma)$ for any non-negative integer $m$ we put $\widehat{m}:=[m / 2]$;

$(\delta)$ as usually, $\left[\begin{array}{c}m \\ n\end{array}\right]_{q}$ stands for the $q$-binomial coefficient.

- Show that polynomials $c_{\Lambda}^{\tau}(q, t)$ have the following property:

$$
c_{\Lambda}^{\tau}(1,1)=\left|\operatorname{Tab}^{(2)}(\Lambda, \tau)\right|,
$$

i.e. $c_{\Lambda}^{\tau}(1,1)$ is equal to the number of semistandard domino tableaux of shape $\Lambda$ and weight $\tau$.

The formula (4.8) with $q=1$ gives very effective method for computing the spin generating function on the set of domino tableaux.

ii) Denote by $\mathbb{S}$ the Schützenberger transformation acting on the set of semistandard Young tableaux of the same shape:

$$
\mathbb{S}: \operatorname{STY}(\lambda, \eta) \rightarrow \operatorname{STY}(\lambda, \overleftarrow{\eta})
$$

where for any composition $\eta=\left(\eta_{1}, \ldots, \eta_{s}\right)$ we denote by $\overleftarrow{\eta}$ the "reverse" composition $\left(\eta_{s}, \eta_{s-1}, \ldots, \eta_{2}, \eta_{1}\right)$. See e.g. [13], Appendix, where the transformation $\mathbb{S}$ is called evacuation, or [30, 35].

Let $\Lambda$ and $\mu$ be partitions such that $|\Lambda|=2|\mu|$, and 2-core $(\Lambda)=\emptyset$.

- Show that there exists a natural bijection

$$
\theta: \operatorname{STY}(\Lambda, \mu \vee \mu)^{\mathbb{S}} \simeq \operatorname{Tab}^{(2)}(\Lambda, \mu)
$$

where for any composition $\mu=\left(\mu_{1}, \ldots, \mu_{p}\right)$ the symbol $\mu \vee \mu$ denotes the composition $\left(\mu_{1}, \mu_{2}, \ldots, \mu_{p-1}, \mu_{p}, \mu_{p}, \mu_{p-1}, \ldots, \mu_{2}, \mu_{1}\right)$. In other words, the set of domino tableaux of shape $\Lambda$ and weight $\mu$ is in one-to-one correspondence with the set of semistandard self-evacuating Young tableaux of shape $\Lambda$ and weight $\mu \vee \mu$. Recall, see e.g., J. Stembridge (Duke Math. J. 82 (1996), 585-606) that a tableau $T \in S T Y(\lambda, \mu)$ is called self-evacuating if $T$ is invariant with respect to the action of Schützenberger's transformation $\mathbb{S}$.

As far as I am aware, the statement that the set of self-evacuating Young tableaux of a given shape $\Lambda$ and weight $\mu \vee \mu$ is equinumerous to that of domino tableaux of the same shape $\Lambda$ and weight $\mu$ was conjectured by $\mathrm{R}$. Stanley and has been proved for the first time 
by J. Stembridge $[i b i d]$. An "elementary proof" has been found later by A.D. Berenstein and A.N. Kirillov (Proc. of 10th Intern. Conf. FPSAC, Fields Institute, Toronto, 1998, p.55-66, and q-alg/9709010). Explicit construction of a bijection between the sets of self-evacuating and domino tableaux was given by S. Fomin (Zap. Nauch. Sem. LOMI 55 (1986), 156-175) in the case $\mu=\left(1^{n}\right)$, and was generalized by A.N. Kirillov, A. Lascoux, B. Leclerc and J.-Y. Thibon (C.R. Acad. Sci. Paris 318 (1994), 395-400) for general $\mu$.

- Show that if a domino tableaux $\mathcal{T} \in \operatorname{Tab}^{(2)}(\Lambda, \mu)$ corresponds to the tableau $T \in$ $\operatorname{STY}(\Lambda, \mu \vee \mu)^{\mathbb{S}}$ under the bijection (4.9) constructed by A.N. Kirillov et al. [ibid], and the semistandard tableau $T$ corresponds to the pair $(\nu ; J)$ under the rigged configurations bijection, then

$$
\operatorname{spin}(\mathcal{T})=\operatorname{spin}(\nu)
$$

where the $\operatorname{spin}(\nu)$ of a configuration $\nu$ is defined by (4.7).

Example 4.7 Take $\lambda=(321), \mu=(3)$ and $\nu=(4321)$. Then $\Lambda=(77411), \tilde{\lambda}=(7321)$, $\widetilde{R}=(43321)$, and $K_{\Lambda, \nu \oplus \nu}(1)=2642, K_{\widetilde{\lambda} \widetilde{R}}(q)=q^{3}(1367531)$. It is not very difficult to check that

$$
\begin{aligned}
& K_{\Lambda, \nu \oplus \nu}(-1)=\left|\operatorname{Tab}^{(2)}(\Lambda, \nu)\right|=K_{\widetilde{\lambda} \widetilde{R}}(1)=26, \\
& |C(\Lambda ; \nu \oplus \nu)|=47, \quad\left|C_{(2)}(\Lambda ; \nu \oplus \nu)\right|=19,
\end{aligned}
$$

and $(q, t)$-domino polynomial is equal to

$$
\begin{aligned}
& q^{4} t+\left[q^{4}+2 q^{5}+2 q^{6}+q^{6}(1+q)\right] t^{2}+\left[3 q^{5}+q^{5}(1+q)+3 q^{6}+q^{6}(1+q)\right. \\
& \left.\quad+q^{9}(1+q)\right] t^{3}+\left[q^{7}(1+q)+2 q^{8}(1+q)\right] t^{4} \\
& =q^{4} t+q^{4} t^{2}[1+q(1+q)(2+q)]+q^{5} t^{3}(1+q)\left[4+q+q^{4}\right] \\
& \quad+q^{7} t^{4}(1+q)[1+2 q] .
\end{aligned}
$$

iii) Let $\lambda$ and $\mu$ be partitions such that 2 -core $(\lambda)=\emptyset$, and $|\lambda|=2|\mu|$.

- Show that for any admissible configuration $\nu \in C(\lambda ; \mu \oplus \mu)$

$$
2 \operatorname{spin}(\nu) \in \mathbb{Z}_{\geq 0} \text { and } 2 \operatorname{spin}(\nu) \equiv c(\nu)(\bmod 2) .
$$

Assume additionally that $\lambda$ has a form $2 \nu \oplus 2 \nu$ for some partition $\nu$, or equivalently, that $2-$ quotient $(\lambda)=(\nu, \nu)$.

- Show that if $\nu \in C_{(2)}(\lambda ; \mu \oplus \mu)$, then

$$
\operatorname{spin}(\nu) \in \mathbb{Z}_{\geq 0}
$$

iv) (Schützenberger transformation and rigged configurations)

Let $\lambda$ be a partition, $R$ be a sequence of rectangular shape partitions, and $T \in L R(\lambda, R)$ be a Littlewood-Richardson tableau. Denote by $\mathbb{S}(T) \in L R(\lambda, \overleftarrow{R})$ the image of tableau $T$ under the Schützenberger transformation, see e.g. 41]. 
- Show that if the pair $\left(\nu,\left\{J_{n, \alpha}^{(k)}\right\}\right)$ corresponds to the tableau $T$ under the rigged configurations bijection, then the pair

$$
\left(\nu,\left\{P_{n}^{(k)}(\nu ; R)-J_{n, m_{n}\left(\nu^{(k)}\right)-\alpha+1}^{(k)}\right\}\right)
$$

corresponds to the tableau $\mathbb{S}(T)$.

This result was discovered and proved by the author in some particular cases (including the case of semistandard tableaux). A proof in general rectangular case has been obtained by A.N. Kirillov, A. Schilling and M. Shimozono 41].

v) Let $\lambda$ and $\mu, l(\mu)=p$, be partitions, and $T \in S T Y(\lambda, \mu)$ be a semistandard Young tableau of shape $\lambda$ and weight $\mu$. With tableau $T$ one can associate a word $w:=w(T)$ which is given by reading the numbers in $T$ from right to left in successive rows starting with the top row. With the word $w:=w(T)$ one can associate a sequence of subwords $w_{1}, w_{2}, \ldots, w_{p}$ such that weight $\left(w_{i}\right)=\left(1^{\left|w_{i}\right|}\right)$ for $i=1, \ldots, p$, see e.g. [52], Chapter III, Section 6, p.242.

Next, let $\nu$ be an admissible configuration corresponding to the tableau $T$ under the rigged configurations bijection, see e.g. [30].

- Show that

$$
\left(\nu^{(1)}\right)_{k}^{\prime}=\partial\left(w_{k}\right), \quad 1 \leq k \leq p,
$$

where for any standard word $w$ the symbol $\partial(w)$ denotes the number of integers $j$ such that $j$ and $j+1$ both appear in the word $w$, but $j+1$ lies to the right of $j$. For example, if $w=5761324$, then $\partial(w)=3$. If $w:=w(T)$, then the numbers $\partial\left(w_{k}\right)$ are related to a structure of descent set $\operatorname{Des}(T)$ of tableau $T$, see e.g. 30.

Recall [29, 30, 35, 41] that the rigged configurations bijection establishes a one-to-one correspondence between the set of Littlewood-Richardson tableaux $L R(\lambda, R)$ and that of rigged configurations $R C(\lambda, R)$. This bijection was discovered and constructed by the author in the middle of 80's, and since that time many interesting combinatorial properties (see e.g. [30, 31, 35] and the present paper) of rigged configurations bijection were found by the author, G.-N. Han, S. Fishel, M. Kleber, A. Schilling and M. Schimozono.

4. Let $p \geq 2$ be an integer, $\lambda$ be a partition such that $p$-core $(\lambda)=\emptyset$, and $R$ be a dominant sequence of rectangular shape partitions such that $|\lambda|=p|R|$. Denote by $R^{(p)}$ a dominant rearrangement of the sequence of rectangular shape partitions $\{\underbrace{R, R, \ldots, R}_{p}\}$.

Denote by $C_{(p)}\left(\lambda ; R^{(p)}\right)$ the subset of the set of admissible configurations $C\left(\lambda ; R^{(p)}\right)$ consisting of all configurations $\nu \in C\left(\lambda ; R^{(p)}\right)$ such that

$$
\left[\frac{P_{n}^{(k)}(\nu ; R)+m_{n}\left(\nu^{(k)}\right)}{p}\right]=\left[\frac{P_{n}^{(k)}(\nu ; R)}{p}\right]+\left[\frac{m_{n}\left(\nu^{(k)}\right)}{p}\right]
$$

for all integers $k, n \geq 1$. Recall that for any $x \in \mathbb{R}$ the symbol $[x]$ stands for the nearest integer which is smaller or equal to $x$.

If $\nu \in C\left(\lambda ; R^{(p)}\right)$, denote by $K_{\nu}(q)$ the following product

$$
K_{\nu}(q)=q^{c(\nu)} \prod_{k, n \geq 1}\left[\begin{array}{c}
P_{n}^{(k)}(\nu ; R)+m_{n}\left(\nu^{(k)}\right) \\
m_{n}\left(\nu^{(k)}\right)
\end{array}\right]_{q},
$$


and if $\nu \in C_{(p)}\left(\lambda ; R^{(p)}\right)$, denote by $K_{\nu}^{(p)}(q)$ the product

$$
K_{\nu}^{(p)}(q)=q^{c(\nu)} \prod_{k, n \geq 1}\left[\begin{array}{c}
\widehat{P}_{n}^{(k)}(\nu ; R)+\widehat{m}_{n}\left(\nu^{(k)}\right) \\
\widehat{m}_{n}\left(\nu^{(k)}\right)
\end{array}\right]_{q},
$$

where for any $m \in \mathbb{Z}$ we denote by $\widehat{m}:=[m / p]$.

It follows from Theorem 4.3 that

$$
K_{\lambda R}(q)=\sum_{\nu \in C\left(\lambda ; R^{(p)}\right)} K_{\nu}(q)
$$

- Show that if $\nu \in C_{(p)}\left(\lambda ; R^{(p)}\right)$, then

$$
K_{\nu}\left(\zeta_{p}\right)= \pm K_{\nu}^{(p)}(1) \in \mathbb{Z}
$$

It looks a challenging task to describe explicitly the sign in the latter formula.

- Show that if $p=2$ or 3 , then the integer numbers $K_{\nu}\left(\zeta_{p}\right)$ have the same sign for all configurations $\nu \in C_{(p)}\left(\lambda ; R^{(p)}\right)$. Note that this is not true if $p \geq 4$.

Conjecture 4.8 Let $\lambda$ and $\mu$ be partitions such that $p-\operatorname{core}(\lambda)=\emptyset$ and $|\lambda|=p|\mu|$. For any configuration $\nu \in C_{(p)}\left(\lambda ; \mu^{(p)}\right)$ one can define the integer number $\operatorname{spin}(\nu)$ which is called by the spin of configuration $\nu$, such that

$$
\sum_{\nu \in C_{(p)}\left(\lambda ; \mu^{(p)}\right)} t^{\operatorname{spin}(\nu)} K_{\nu}^{(p)}(1)=\epsilon_{p}(\lambda) \sum_{T \in T_{a b^{(p)}}(\lambda, \mu)} t^{\operatorname{spin}(T)},
$$

where $\epsilon_{p}(\lambda)= \pm 1$, and $\mu^{(p)}=\mu \oplus \cdots \oplus \mu$ ( $p$ times $)$.

5. Let $\lambda, \mu=\left(\mu_{1} \geq \cdots \geq \mu_{p}>0\right)$ and $\nu$ be partitions such that $|\nu|=|\lambda|+|\mu|$. In the sequel we will identify a partition $\lambda$ with its diagram, see e.g. [52], Chapter I, p.2.

Let $x_{1}$ be the rightmost square in the top row of $\lambda$, and let $x_{2}$ be the leftmost square in the bottom row of $\mu$. Let $\mu^{v}$ (respectively, $\mu^{h}$ ) denote the diagram obtained from $\mu$ by a shift sending $x_{2}$ to the square immediately above $x_{1}$ (respectively, immediately right of $x_{1}$ ) and let $\lambda * \mu^{v}$ (respectively, $\lambda * \mu^{h}$ ) denote the diagram $\lambda \cup \nu^{v}$ (respectively, $\lambda \cup \mu^{h}$ ). Finally, let $\lambda * \mu$ denote the partition $\left(\left(\lambda_{1}^{p}\right)+\mu\right) \oplus \lambda$ and $\widetilde{\nu}$ (respectively, $\widetilde{\nu}_{h}$ and $\widetilde{\nu}_{v}$ ) denote a dominant rearrangement of the sequence of rectangular shape partitions $\left(\left(\lambda_{1}^{p}\right), \nu\right)$ (respectively, $\left(\left(\lambda_{1}-\right.\right.$ $\left.\left.1)^{p}\right), \nu\right)$ and $\left(\left(\lambda_{1}^{p-1}\right), \nu\right)$.

Show that

$$
K_{\lambda * \mu, \widetilde{\nu}}(q)=K_{\lambda * \mu^{h}, \widetilde{\nu}_{h}}(q)+q^{\alpha(\lambda, \mu, \nu)} K_{\lambda * \mu^{v}, \widetilde{\nu}_{v}}(q),
$$

where $\alpha(\lambda, \mu, \nu)$ is a non-negative integer which may be computed explicitly.

The latter relation (4.15) between parabolic Kostka polynomials may be considered as a $q$-analog of the formula (1) from [52], Chapter I, Section 5, Exercise 21(a), in the case when both $\theta$ and $\varphi$ (see $[i b i d])$ are partitions. 
Note finally, see Section [2, Exercise 3 ii), that

$$
K_{\lambda * \mu, \widetilde{\nu}}(1)=\left|\operatorname{Tab}^{(2)}(\Lambda, \nu)\right|,
$$

where $\Lambda$ is a unique partition such that 2 -core $(\Lambda)=\emptyset$ and 2 -quotient $(\Lambda)=(\lambda, \mu)$. It will be interesting to find an explanation of the relation (4.15) based on consideration domino tableaux only.

6. (Examples of fewnomial parabolic Kostka polynomials)

a. Let $n \geq 2$ be an integer. Consider almost staircase partition

$$
\lambda=(2 n, 2 n-1, \ldots, n, \widehat{n-1}, n-2, \ldots, 2,1),
$$

and sequence of rectangular shape partitions

$$
R=\left((2 n-1),\left(n^{n}\right),\left((n-1)^{n-1}\right),(1)\right) .
$$

i) Show that

$$
K_{\lambda R}(q)=(n-1) q\left(\begin{array}{c}
n \\
2
\end{array}\right)+1(1+q)^{2} .
$$

ii) Let $k \geq 1$ be an integer. Consider partition

$$
\lambda^{(k)}=\lambda \oplus \underbrace{(2 n-1) \oplus \cdots \oplus(2 n-1)}_{k},
$$

and sequence of rectangular shape partitions

$$
R^{(k)}=(\underbrace{(2 n-1), \ldots,(2 n-1)}_{k+1},\left(n^{n}\right),\left((n-1)^{n-1}\right),(1)) .
$$

Show that

$$
\begin{aligned}
K_{\lambda^{(k)}, R^{(k)}}(q) & =q^{\left(\begin{array}{c}
n \\
2
\end{array}\right)+1}\left[(n-1)+3(n-1) q+3 n \frac{q^{2}-q^{k+1}}{1-q}\right. \\
& \left.+(3 n-4) q^{k+1}+(n-1) q^{k+2}\right] .
\end{aligned}
$$

In particular, $\lim _{k \rightarrow \infty} K_{\lambda^{(k)}, R^{(k)}}(q) \doteq \frac{(n-1)+2(n-1) q+3 q^{2}}{1-q}$.

b. Let $n \geq 1$ be an integer. Consider almost staircase partition

$$
\lambda=(2 n+1,2 n, \ldots, n+1, \widehat{n}, n-1, \ldots, 2,1),
$$

and sequence of rectangular shape partitions

$$
R=\left((2 n),\left((n+1)^{n}\right),\left(n^{n-1}\right),(1)\right) .
$$


Show that

$$
K_{\lambda R}(q)=q^{\left(\begin{array}{c}
n \\
2
\end{array}\right)+1}(1+q)(n+(n-1) q) .
$$

c. Let $n \geq 1$ be an integer, and $\lambda$ be the almost staircase partition from $\mathbf{b}$. Consider a sequence of rectangular shape partitions $R=\left((2 n),\left(n^{n}\right),\left(n^{n}\right),(1)\right)$.

Show that

$$
K_{\lambda R}(q)=q^{\left(\begin{array}{c}
n \\
2
\end{array}\right)+1}\left(n+(2 n-1) q+n q^{2}\right) .
$$

d. Let $n \geq 2$ be an integer. Consider almost staircase partition

$$
\lambda=(2 n, \widehat{2 n-1}, 2 n-2, \ldots, n, \widehat{n-1}, n-2, \ldots, 2,1)
$$

and sequence of rectangular shape partitions

$$
R=\left((2 n-1),\left((n-1)^{n-1}\right),\left((n-1)^{n-1}\right),(1)\right) .
$$

i) Show that

$$
K_{\lambda R}(q)=q^{\left(\begin{array}{c}
n \\
2
\end{array}\right)+1}(n-1,2 n-3, n-1) .
$$

ii) Let $k \geq 1$ be an integer. Consider partition

$$
\lambda^{(k)}=\lambda \oplus \underbrace{(2 n-1) \oplus(2 n-1) \cdots \oplus(2 n-1)}_{k},
$$

and sequence of rectangular shape partitions

$$
R^{(k)}=(\underbrace{(2 n-1), \ldots,(2 n-1)}_{k+1},\left((n-1)^{n-1}\right),\left((n-1)^{n-1}\right),(1)) .
$$

Show that

$$
\begin{aligned}
K_{\lambda^{(k)}, R^{(k)}}(q) & =q^{\left(\begin{array}{c}
n \\
2
\end{array}\right)+1}\left[(n-1)+(3 n-4) q+3 n \frac{q^{2}-q^{k+1}}{1-q}\right. \\
& \left.+(3 n-4) q^{k+1}+(n-1) q^{k+2}\right] .
\end{aligned}
$$

In particular, $\lim _{k \rightarrow \infty} K_{\lambda^{(k)}, R^{(k)}}(q) \doteq \frac{(n-1)+(2 n-3) q+4 q^{2}}{1-q}$.

e. Let $n \geq 3$ be an integer. Consider staircase partition

$$
\lambda=(2 n+1,2 n, \ldots, 2,1)
$$

and a sequence of rectangular shape partitions

$$
R=\left((2 n),(2 n-1,2 n-1),\left((n+1)^{n-1}\right),\left((n-1)^{n-2}\right),(2)\right) .
$$


Show that

$$
K_{\lambda R}(q)=2(n-2) q\left(\begin{array}{c}
n-2 \\
2
\end{array}\right)+2(1+q)^{3} .
$$

f. i) Let $\rho_{n}=(2 n-1,2 n-3, \ldots, 3,1)$ be a partition with parts which are consecutive odd numbers, starting from 1 till $2 n-1$. Consider partition $\lambda=\rho_{n} \oplus(2 n) \oplus(2 n)$, and a sequence of rectangular shape partitions

$$
R=\left((2 n-1),(\underbrace{n, \ldots, n}_{\left[\frac{n+3}{2}\right]}),(\underbrace{n, \ldots, n}_{\left[\frac{n+2}{2}\right]}),(1)\right) .
$$

Show that

$$
K_{\lambda R}(q)=q^{\left[\frac{n+2}{2}\right]\left[\frac{n+3}{2}\right]}\left(\left[\frac{n+2}{2}\right], n,\left[\frac{n+2}{2}\right]\right) .
$$

ii) Consider partition $\lambda=\rho_{n} \oplus(2 n+1) \oplus(2 n)$, and a sequence of rectangular shape partitions

$$
R=\left((2 n),(\underbrace{n, \ldots, n}_{\left[\frac{n+3}{2}\right]}),(\underbrace{n, \ldots, n}_{\left[\frac{n+2}{2}\right]}),(1)\right)
$$

Show that

$$
K_{\lambda R}(q)=q^{\left[\frac{n+2}{2}\right]\left[\frac{n+3}{2}\right]}\left(\left[\frac{n+2}{2}\right], n+1,\left[\frac{n+2}{2}\right]\right) .
$$

iii) Let $\widetilde{\rho}_{n}$ denotes partition $\rho_{n}+(\underbrace{0, \ldots, 0}_{n-1}, 1)$. Consider partition $\lambda=\widetilde{\rho}_{n} \oplus(2 n) \oplus(2 n)$, and a sequence of rectangular shape partitions

$$
R=\left((2 n-1),(\underbrace{n, \ldots, n}_{\left[\frac{n+3}{2}\right]}),(\underbrace{n, \ldots, n}_{\left[\frac{n+2}{2}\right]}),(2)\right) .
$$

Show that if $n \geq 3$, then

$$
K_{\lambda R}(q)=q^{\left[\frac{n+2}{2}\right]\left[\frac{n+3}{2}\right]}\left(\left[\frac{n+2}{2}\right],\left[\frac{3 n}{2}\right],\left[\frac{3 n}{2}\right],\left[\frac{n+2}{2}\right]\right) .
$$

iv) Let $\lambda$ be the same partition as in iii). Consider a sequence of rectangular shape partitions

$$
R=\left((2 n-1),(\underbrace{n, \ldots, n}_{\left[\frac{n+3}{2}\right]}),(\underbrace{n, \ldots, n}_{\left[\frac{n+2}{2}\right]}),(1,1)\right) .
$$

Show that if $n \geq 2$, then

$$
K_{\lambda R}(q)=q^{\left[\frac{n+2}{2}\right]\left[\frac{n+3}{2}\right]}\left(\left[\frac{n}{2}\right],\left[\frac{3 n-2}{2}\right],\left[\frac{3 n-2}{2}\right],\left[\frac{n}{2}\right]\right) .
$$




\section{Generalized exponents and mixed tensor represen- tations}

Let $\mathfrak{g}=\operatorname{sl}(N, \mathbb{C})$ denote the Lie algebra of all $N \times N$ complex matrices of trace 0 , and $G=G L(N, \mathbb{C})$ denote the Lie group of all invertible $N \times N$ complex matrices. Let

$$
a d: G \rightarrow \operatorname{Aut}(\mathfrak{g})
$$

denote the adjoint representation of $G$, defined by

$$
(\operatorname{adX})(A)=X A X^{-1}
$$

where $X \in G$, and $A \in \mathfrak{g}$.

The adjoint action of $G L(N, \mathbb{C})$ extends to the action on the symmetric algebra

$$
S^{\bullet}(\mathfrak{g})=\oplus_{k \geq 0} S^{k}(\mathfrak{g}),
$$

where $S^{k}(\mathfrak{g})$ denotes the $k$-th symmetric power. It is well known 42 that the ring

$$
I=S^{\bullet}(\mathfrak{g})^{G}=\left\{f \in S^{\bullet}(\mathfrak{g}) \mid X \cdot f=f, \forall x \in G\right\}
$$

of invariants of this action is a polynomial ring in $N-1$ variables $f_{2}, \ldots, f_{N-1}$, where $f_{i} \in$ $S^{i}(\mathfrak{g})^{G}$. By a theorem of Kostant 42,

$$
S^{\bullet}(\mathfrak{g})=I \otimes H
$$

is a free module over $G$-invariants $I$ generated by harmonics $H$. Moreover,

$$
H=\oplus_{p \geq 0} H^{p}
$$

is a graded (so $H^{p}=H \cap S^{p}(\mathfrak{g})$ ), locally finite $\mathfrak{g}$-representation. The graded character $\mathrm{ch}_{q}$ of the symmetric algebra of adjoint representation is given by the following formal power series

$$
\operatorname{ch}_{q}\left(S^{\bullet}(\mathfrak{g})\right)=\sum_{k \geq 0} q^{k} \operatorname{ch}\left(S^{k}(\mathfrak{g})\right)=\prod_{1 \leq i, j \leq n}\left(1-q x_{i} / x_{j}\right)^{-1}
$$

For any finite dimensional $\mathfrak{g}$-representation $V$ let us define

$$
G_{N}(V):=G_{N}(V ; q)=\sum_{p \geq 0}\left\langle V, H^{p}\right\rangle q^{p}
$$

where

$$
\left\langle V_{1}, V_{2}\right\rangle=\operatorname{dim}_{\mathfrak{g}} \operatorname{Hom}\left(V_{1}, V_{2}\right)
$$

is the standard pairing on the representation ring of the Lie algebra $\mathfrak{g}$. By a theorem of Kostant 42,

$$
\left.G_{N}(V)\right|_{q=1}=\operatorname{dim} V(0)
$$


where $V(0)$ denotes the zero weight subspace of representation $V$. Hence, $G_{N}(V)$ is a polynomial in $q$ with non-negative integer coefficients. Follow Kostant [42], the integers $e_{1}, \ldots, e_{s}$ with

$$
G_{n}(V)=\sum_{i=1}^{s} q^{e_{i}}
$$

are called generalized exponents of the representation $V$. Kostant's problem $[$ ibid] is to determine/compute these numbers for a given representation $V$.

Let $V_{\lambda}:=V_{\lambda}^{[N]}$ denotes the irreducible highest weight $\lambda$ representation of the Lie algebra $\mathfrak{g}:=\operatorname{sl}(N, \mathbb{C})$. Theorem 5.1 below together with the fermionic formula (4.2) for the KostkaFoulkes polynomials, gives an effective method for computing the generalized exponents of irreducible representation of the Lie algebra $\mathfrak{g}=\operatorname{sl}(N)$.

Theorem 5.1 (20]) Let $\lambda$ be a partition, then

$$
G_{N}\left(V_{\lambda}\right)= \begin{cases}K_{\lambda,\left(\left(\frac{|\lambda|}{N}\right)^{N}\right)}(q), & \text { if }|\lambda| \equiv 0(\bmod N), \\ 0, & \text { otherwise. }\end{cases}
$$

For an "elementary" proof of Theorem 5.1, which is based only on the theory of symmetric functions, see [10].

Using Theorem 5.1, one can compute, in principal, the generalized exponents for any finite dimensional $\mathfrak{g} l(N)$-module $V$. What seems to be very interesting is that for certain representations, see below, there exist alternative expressions for the generalized exponents polynomials which have independent interest and more convenient for computations. Before turning to our main results of this Section, it is useful to recall a few definitions and results from [3, 4, 20].

Let $\alpha, \beta$ be partitions, and $V_{\alpha}^{(N)}, V_{\beta}^{(N)}$ be the highest weight $\alpha$ and $\beta$ (respectively) irreducible representations of the Lie algebra $\mathfrak{g} l(N)$. For any finite dimensional $\mathfrak{g} l(N)$-module $V$ let $V^{*}$ denote its dual. If $l(\alpha)+l(\beta) \leq N$, denote by $V_{\alpha, \beta}^{(N)}$ the Cartan piece in the tensor product $V_{\alpha}^{(N)} \otimes V_{\beta}^{(N) *}$, i.e. the irreducible submodule generated by the tensor product of highest weight vectors of each component. Follow [3, 20], we call a representation obtained in this way by mixed tensor representation. Clearly, $V_{\alpha, \beta}^{(N)}$ is the dual of $V_{\beta, \alpha}^{(N)}$.

Since it is irreducible, $V_{\alpha, \beta}^{(N)}$ is equal to $V_{\lambda}^{(N)}$ for a unique partition $\lambda$ of less than $N$ rows. Let us write $[\alpha, \beta]_{N}$ for this $\lambda$. It is well-known [3, 20], and goes back to D.E. Littlewood 46], that

$$
[\alpha, \beta]_{N}=(\alpha_{1}+\beta_{1}, \ldots, \alpha_{s}+\beta_{1}, \underbrace{\beta_{1}, \ldots, \beta_{1}}_{N-s-r}, \beta_{1}-\beta_{r}, \ldots, \beta_{2}-\beta_{1}, 0),
$$

where $s=l(\alpha), r=l(\beta)$, and we assume that $s+r \leq N$. For example,

$$
V_{0,0}^{(N)}=V_{0}^{(N)} \simeq \mathbb{C}, \quad V_{(1),(1)}^{(N)}=V_{\left(21^{N-2}\right)}^{(N)}=\mathfrak{g}
$$

is the adjoint representation. 
Theorem $5.2([3,20])$ i) Fix $N \geq 1$. Then the representations $V_{\alpha \beta}^{[N]}$, where partitions $\alpha$ and $\beta$ satisfy $l(\alpha)+l(\beta) \leq N$, and $|\alpha|=|\beta|$, form a complete, repetition-free list of the irreducible finite-dimensional representations of the group $P G L_{N}$;

ii) $G_{N}\left(V_{\alpha, \beta}\right)=K_{[\alpha, \beta]_{N},\left(\beta_{1}^{N}\right)}(q)$;

iii) $G_{N}\left(V_{\alpha} \otimes V_{\beta}^{*}\right):=G_{N}\left(V_{\alpha}^{[N]} \otimes\left(V_{\beta}^{[N]}\right)^{*}\right)$

$=\sum_{\mu} K_{\alpha \mu}(q) K_{\beta \mu}(q)\left[\begin{array}{c}N \\ N-\mu_{1}^{\prime}, \mu_{1}^{\prime}-\mu_{2}^{\prime}, \ldots, \mu_{N}^{\prime}\end{array}\right]_{q}$, where $\mu_{i}^{\prime}, 1 \leq i \leq N$, denote the column lengths of partition $\mu$, and

$$
\left[\begin{array}{c}
N \\
m_{1}, \ldots, m_{N}
\end{array}\right]_{q}=\frac{(q ; q)_{N}}{(q ; q)_{m_{1}} \cdots(q ; q)_{m_{N}}}
$$

denotes the q-multinomial coefficient if $m_{i} \geq 0,1 \leq i \leq N, N=m_{1}+\cdots+m_{N}$, and 0 otherwise.

Our first result in this Section is Theorem 5.3 below, which connects the generalized exponents polynomial $G_{N}\left(V_{\alpha} \otimes V_{\beta}^{*}\right)$ with a certain parabolic Kostka polynomial, and gives, via Corollary 5.4, the first real means for computing the $G_{N}\left(V_{\alpha} \otimes V_{\beta}^{*}\right)$.

Theorem 5.3 Let $\alpha, \beta$ be partitions, $|\alpha|=|\beta|, l(\alpha) \leq r$. Then

$$
G_{N}\left(V_{\alpha} \otimes V_{\beta}^{*}\right) \doteq K_{[\alpha, \beta]_{N+r}, R_{N}}(q)
$$

where $R_{N}=\{\underbrace{\left(\beta_{1}\right), \ldots,\left(\beta_{1}\right)}_{N},\left(\beta_{1}^{r}\right)\}$.

Corollary 5.4 (Fermionic formula for the generalized exponents polynomial $G_{N}\left(V_{\alpha} \otimes V_{\beta}^{*}\right)$ ) Let $\alpha, \beta$ be partitions, $|\alpha|=|\beta|, l(\alpha) \leq r$. Then

$$
q^{|\alpha|} G_{N}\left(V_{\alpha} \otimes V_{\beta}^{*}\right)=\sum_{\nu} q^{c(\nu)} \prod_{k, j \geq 1}\left[\begin{array}{c}
P_{j}^{(k)}(\nu)+m_{j}\left(\nu^{(k)}\right)+k \delta_{j, \beta_{1}} \theta(r-k) \\
P_{j}^{(k)}(\nu)
\end{array}\right]_{q}
$$

summed over all admissible configurations $\nu$ of type $\left([\alpha, \beta]_{N} ;\left(\beta_{1}^{N}\right)\right)$.

See Definitions 4.1 and 4.2 for explanation of symbols in the RHS(5.3)

Remark 5.5 Let $\alpha, \beta$ be partitions such that $l(\alpha)+l(\beta) \leq N, l(\alpha) \leq r$. Then

$$
G_{N}\left(V_{\alpha} \otimes V_{\beta}^{*}\right) \neq 0
$$

if and only if $|\alpha| \equiv|\beta| \bmod N$ and $\widetilde{\beta}_{1}=\beta_{1}+\frac{|\alpha|-|\beta|}{N} \geq 0$;

if so, then

$$
G_{N}\left(V_{\alpha} \otimes V_{\beta}^{*}\right)=K_{[\alpha, \beta]_{N+r}, \widetilde{R}_{N}}(q),
$$


where $\widetilde{R}_{N}=\{\underbrace{\left(\widetilde{\beta}_{1}\right), \ldots,\left(\widetilde{\beta}_{1}\right)}_{N},\left(\beta_{1}^{r}\right)\}$.

Also we have

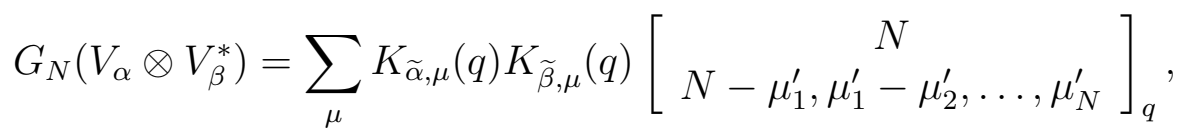

where $\widetilde{\alpha}=\alpha$, and $\widetilde{\beta}=\beta \oplus\left(\left(\frac{|\alpha|-|\beta|}{N}\right)^{N}\right)$, if $|\alpha| \geq|\beta|$, and $\widetilde{\alpha}=\alpha \oplus\left(\left(\frac{|\beta|-|\alpha|}{N}\right)^{N}\right)$, and $\widetilde{\beta}=\beta$, if $|\alpha| \leq|\beta|$.

Corollary 5.6 Let $\alpha, \beta$ be partitions, $|\alpha|=|\beta|$, then

$$
\sum_{\mu} \frac{K_{\alpha \mu}(q) K_{\beta \mu}(q)}{b_{\mu}(q)}=\frac{K_{\beta \alpha}(q, q)}{H_{\alpha}(q)}
$$

where $K_{\beta \alpha}(q, q)$ denotes the specialization $q=t$ of the double Kostka polynomial $K_{\beta \alpha}(q, t)$ introduced by Macdonald [52], Chapter VI, §8;

$$
b_{\mu}(q):=\prod_{i \geq 1}(q ; q)_{\mu_{i}^{\prime}-\mu_{i+1}^{\prime}} .
$$

Proof. It follows from Theorem 5.2, iii) that the

$$
\operatorname{LHS}(5.3)=\lim _{N \rightarrow \infty} G_{N}\left(V_{\alpha} \otimes V_{\beta}^{*}\right)
$$

If we compare the RHS(5.3) with that of (6.14), we immediately see that

$$
\lim _{N \rightarrow \infty} G_{N}\left(V_{\alpha} \otimes V_{\beta}^{*}\right)=q^{-|\alpha|} \lim _{N \rightarrow \infty} s_{\alpha} * s_{\beta}\left(q, \ldots, q^{N-1}\right)=s_{\alpha} * s_{\beta}\left(1, q, q^{2}, \ldots\right),
$$

where $s_{\alpha} * s_{\beta}(1, q, \ldots)$ denotes the principal specialization $x_{i}=q^{i-1}, 1,2,3, \ldots$, of the internal product of Schur functions $s_{\alpha}$ and $s_{\beta}$, see Section 6 for definition. It follows from [52], Chapter VI, §8, Example 3, that

$$
s_{\alpha} * s_{\beta}\left(1, q, q^{2}, \ldots\right)=\frac{K_{\alpha \beta}(q, q)}{H_{\beta}(q)}=\frac{K_{\beta \alpha}(q, q)}{H_{\alpha}(q)} .
$$

Remark 5.7 The identity (5.6) is essentially equivalent to the definition of Kostka-Foulkes polynomials $K_{\lambda \mu}(q)$, see Section 2 , (2.1). Indeed, by definition,

$$
s_{\beta}(x)=\sum_{\mu} K_{\beta \mu}(q) P_{\mu}(x ; q)=\sum_{\mu} \frac{K_{\beta \mu}(q)}{b_{\mu}(q)} Q_{\mu}(x ; q) .
$$


Therefore, in the $\lambda$-ring notation, we have

$$
s_{\beta}\left[\frac{X}{1-q}\right]=\sum_{\mu} \frac{K_{\beta \mu}(q)}{b_{\mu}(q)} Q_{\mu}\left[\frac{X}{1-q} ; q\right] .
$$

By definition,

$$
Q_{\mu}\left[\frac{X}{1-q} ; q\right]=Q_{\mu}^{\prime}(x ; q)
$$

is the modified Hall-Littlewood polynomial, and

$$
Q_{\mu}^{\prime}(x ; q)=\sum_{\alpha} K_{\alpha \mu}(q) s_{\alpha}(x) .
$$

On the other hand, by definition (see [52], Chapter VI, (8.11))

$$
J_{\mu}(x ; q, t)=\sum_{\lambda} K_{\lambda \mu}(q, t) s_{\lambda}[X(1-t)]
$$

or

$$
J_{\mu}\left[\frac{X}{1-t} ; q, t\right]=\sum_{\lambda} K_{\lambda \mu}(q, t) s_{\lambda}(x) .
$$

Now, if we put $q=t$ in (5.8), we obtain

$$
H_{\mu}(q) s_{\mu}\left[\frac{X}{1-q}\right]=\sum_{\lambda} K_{\lambda \mu}(q, q) s_{\lambda}(x) .
$$

Hence,

$$
\sum_{\alpha} \frac{K_{\alpha \beta}(q, q)}{H_{\beta}(q)} s_{\alpha}(x)=s_{\beta}\left[\frac{X}{1-q}\right]=\sum_{\alpha} \sum_{\mu} \frac{K_{\beta \mu}(q) K_{\alpha \mu}(q)}{b_{\mu}(q)} s_{\alpha}(x) .
$$

These equalities imply (5.6).

We conclude this Section with a generalization of Theorem 5.1.

By a theorem of Kostant [42] the symmetric algebra $S^{\bullet}(\mathfrak{g})$ of the adjoint representation $\mathfrak{g}$ of the Lie algebra $\mathfrak{g} l(N)$ is isomorphic as the graded $\mathfrak{g} l(N)$-modules to the direct sum of modules $V_{\mu} \otimes V_{\mu}^{*}$ when $\mu$ ranges over the set $\mathcal{P}_{N}$ of all partitions whose length do not exceed $N$ :

$$
S^{\bullet}(\mathfrak{g}) \simeq \oplus_{\mu \in \mathcal{P}_{N}}\left(V_{\mu} \otimes V_{\mu}^{*}\right) .
$$

Let $l \geq 2$ be an integer, we are going to introduce and study $l$-restricted generalized exponents. Consider the algebra of $l$-restricted representations of the $q$-analog of universal enveloping algebra $U_{q}(\operatorname{sl}(N))$, when $q=\exp \left(\frac{2 \pi i}{l+N}\right)$ is the primitive $(l+N)$-th root of unity. This algebra, known as Verlinde algebra $\mathcal{V}(l, N)$, has generators $V_{\lambda}$, where $\lambda$ ranges over the set $\mathcal{P}_{N}^{(l)}$ of $l$-restricted partitions, i.e. partitions $\lambda$ such that $l(\lambda)<N, l\left(\lambda^{\prime}\right) \leq l$. The multiplication in the algebra $\mathcal{V}(l, N)$ is given by the so-called $l$-restricted tensor product, which we will denote by $\widehat{\otimes}$. We refer the reader to [27], Chapter 13, Exercise 13.34, for definition and basic properties of the Verlinde algebra. 
Definition 5.8 Let $l \geq 2$ be an integer, define

$$
\begin{aligned}
S_{l}^{\bullet}(\mathfrak{g}) & =\bigoplus_{k \geq 0} S_{l}^{k}(\mathfrak{g}), \quad \text { where } \\
S_{l}^{k}(\mathfrak{g}) & =\bigoplus_{|\mu|=k}\left(V_{\mu} \widehat{\otimes} V_{\mu}^{*}\right)
\end{aligned}
$$

and $\mu$ ranges over the set $\mathcal{P}_{N}^{(l)}$ of l-restricted partitions, and $|\mu|=k$.

Definition 5.9 (l-restricted generalized exponents) Let $V$ belongs to the Verlinde algebra $\mathcal{V}(l, N)$. The l-restricted generalized exponents polynomial $G_{N}^{(l)}(V)$ is defined by the following formula

$$
G_{N}^{(l)}(V):=G_{N}^{(l)}(V ; q)=\sum_{k \geq 0}\left[V: S_{l}^{k}(\mathfrak{g})\right] q^{k},
$$

where for any two elements $V$ and $W$ of the Verlinde algebra $\mathcal{V}(l, N)$ the symbol $[V: W]$ has the following meaning:

let $V=\sum_{\lambda} a_{\lambda} V_{\lambda}$ and $W=\sum_{\mu} b_{\mu} V_{\mu}$ be the decompositions of the elements $V$ and $W$ in terms of the generators $V_{\lambda}$ of the Verlinde algebra $\mathcal{V}(l, N)$. By definition,

$$
[V: W]=\sum_{\lambda} a_{\lambda} b_{\lambda}
$$

Definition 5.10 (Verlinde polynomials) Let $l \geq 2$ and $L \geq 1$ be integers, and $V \in \mathcal{V}(l, N)$. Denote by $\left(S_{l}^{\bullet}(\mathfrak{g})^{\widehat{\otimes} L}\right)^{(k)}$ the degree $k$ component of the $L$-th $l$-restricted tensor power of the element $S_{l}^{\bullet}(\mathfrak{g}) \in \mathcal{V}(l, N)$. Polynomial

$$
G_{N}^{(l, L)}(V):=\sum_{k \geq 0}\left[V:\left(S_{l}^{\bullet}(\mathfrak{g})^{\widehat{\otimes} L}\right)^{(k)}\right] q^{k}
$$

is called by Verlinde polynomial.

Let us denote by $\widehat{\mathcal{P}}_{N}^{(l)}$ the set of all partitions $\lambda$ such that $l(\lambda) \leq N$ and $\lambda_{1}-\lambda_{N} \leq l$.

Problem 5.11 Let $\lambda, \alpha, \beta$ be partitions from the set $\widehat{\mathcal{P}}_{N}^{(l)}$. Compute polynomials $G_{N}^{(l, L)}\left(V_{\lambda}\right)$ and $G_{N}^{(l, L)}\left(V_{\alpha} \widehat{\otimes} V_{\beta}^{*}\right)$.

Theorem 5.12 Let $\lambda \in \widehat{\mathcal{P}}_{N}^{(l)}$, then

$$
G_{N}^{(l)}\left(V_{\lambda}\right)= \begin{cases}K_{\lambda,\left(\left(\frac{|\lambda|}{N}\right)^{N}\right)}^{(l)}(q), & \text { if }|\lambda| \equiv 0(\bmod N) \\ 0, & \text { otherwise }\end{cases}
$$


Here for a partition $\lambda \in \widehat{\mathcal{P}}_{n}^{(l)}$ and any partition $\mu$ the symbol $K_{\lambda, \mu}^{(l)}(q)$ denotes the $l-$ restricted Kostka-Foulkes polynomial. We refer the reader to [26], Section 4.1, see also references therein, for a definition of the so-called l-restricted one dimensional sums $X_{\mu}^{(l)}(\lambda)$ which we identify with $l$-restricted Kostka-Foulkes polynomials

$$
K_{\lambda \mu}^{(l)}(q):=X_{\mu}^{(l)}(\lambda)
$$

In my opinion, this is one of the most "natural" definitions of the $l$-restricted Kostka-Foulkes polynomials. Other definitions appeal either to the Representation Theory or Quantum Groups at roots of unity [folklore], or that of the Hecke-Iwahori algebras (Goodman F. and Wenzl H., Adv. Math. 82 (1990), 244-265). Restricted Kostka-Foulkes polynomials have many interesting combinatorial properties (restricted Young tableaux, restricted LittlewoodRichardson rule, ...), and appear to be connected with the characters of Virasoro and affine Lie algebras, see e.g. [33], p.101-105; [26], Section 5, and references therein.

Problem 5.13 Let $\alpha$ and $\beta$ be partitions of the same size from the set $\widehat{\mathcal{P}}_{N}^{(l)}$. Define lrestricted double-Kostka polynomial $K_{\alpha \beta}^{(l)}(q, t)$ with the following properties

i) $K_{\alpha \beta}^{(l)}(q, t)$ is a polynomial with non-negative integer coefficients;

ii) $K_{\alpha \beta}^{(l)}(0, t)$ is equal to the l-restricted Kostka-Foulkes polynomial $K_{\alpha \beta}^{(l)}(t)$;

iii) if $l$ is big enough, then $K_{\alpha \beta}^{(l)}(q, t)$ coincides with the double Kostka polynomial $K_{\alpha \beta}(q, t)$ introduced by Macdonald [52], Chapter VI.

See recent preprint by L. Lapointe, A. Lascoux and J. Morse, A filtration of the symmetric function space and refinement of the Macdonald positivity conjecture, math.QA/0008073, where certain polynomials $K_{\lambda \mu}^{(k)}(q, t) \leq K_{\lambda \mu}(q, t)$ which might be satisfy the above conditions i) and ii), have been introduced and studied.

\section{Exercises}

1. Show that if $\beta=(n)$, then the identity (5.6) takes the following form

$$
\sum_{\mu \vdash n} \frac{q^{n(\mu)} K_{\alpha, \mu}(q)}{b_{\mu}(q)}=\frac{K_{\alpha^{\prime},\left(1^{n}\right)}(q)}{(q ; q)_{n}} .
$$

In the particular case $\alpha=(n)$, the identity (5.9) becomes

$$
\sum_{\mu \vdash n} \frac{q^{2 n(\mu)}}{\prod_{i}(q ; q)_{m_{i}(\mu)}}=\frac{1}{(q ; q)_{n}} .
$$

This identity is due to Philip Hall (Comm. Math. Helv. 11 (1938), 126-129).

2. Let $\alpha$ be a partition of $n$. Show that

$$
\sum_{\mu \vdash n} \frac{q^{n(\mu)} K_{\alpha, \mu}(q) \prod_{j=1}^{\mu_{1}^{\prime}}\left(1+z q^{1-j}\right)}{b_{\mu}(q)}=\prod_{(i, j) \in \alpha}\left(1+z q^{j-i}\right) \frac{K_{\alpha^{\prime},\left(1^{n}\right)}(q)}{(q ; q)_{n}} .
$$


In the particular case $\alpha=(n)$, we obtain a generalization of the Philip Hall identity (5.10):

$$
\sum_{\mu \vdash n} \frac{q^{2 n(\mu)} \prod_{j=1}^{\mu_{1}^{\prime}}\left(1+z q^{1-j}\right)}{b_{\mu}(q)}=\frac{(-z ; q)_{n}}{(q ; q)_{n}} .
$$

3. Fusion rules and Catalan numbers.

Let $q^{n+2}=1, n \equiv 1(\bmod 2)$ be a primitive root of 1 of odd order. For each integer $m$ such that $0 \leq 2 m+1 \leq n$, denote by $V_{m}$ the $(2 m+1)$-dimensional irreducible representation of $U_{q}(\operatorname{sl}(2))$. Show that

$$
\begin{aligned}
& \text { Mult }\left[V_{0}: V_{\frac{n-1}{2}}^{\widehat{\otimes} 2 k}\right]=C_{k}, \quad \text { if } k \leq n-2, \\
& \operatorname{Mult}\left[V_{0}: V_{\frac{n-1}{2}}^{\widehat{\otimes} 2 k}\right]<C_{k}, \quad \text { if } \quad k \geq n-1 .
\end{aligned}
$$

Here $C_{k}$ denotes the $k$-th Catalan number. In other words, if $n \geq k+2$, then the Catalan number $C_{k}$ is equal to the $(n-2)-$ restricted Kostka number $K_{\lambda \mu}^{(n-2)}$, where $\lambda=(k(n-$ $1) / 2, k(n-1) / 2)$ and $\mu=\left(((n-1) / 2)^{2 k}\right)$.

Using the fermionic formula (3.13) for restricted Kostka polynomials, show that

$$
C_{k}=\sum_{\nu} \prod_{j \geq 1}\left(\begin{array}{c}
P_{j}(\nu)+m_{j}(\nu) \\
m_{j}(\nu)
\end{array}\right),
$$

summed over all partitions $\nu$ such that

- $|\nu|=k(n-1) / 2, \nu_{1} \leq n-2$,

- $P_{j}(\nu):=k \min (2 j, n-1)-2 Q_{j}(\nu) \geq 0$.

Thus, if $n \geq k+2$, then $(n-2)$-restricted Kostka polynomial $K_{\lambda \mu}^{(n-2)}(q)$ with $\lambda=$ $\left((k(n-1) / 2)^{2}\right)$ and $\mu=\left(((n-1) / 2)^{2 k}\right)$, see (3.13), may be considered as a $q$-analog of the Catalan number $C_{k}$.

4. Generalized exponents for the Lie algebras of type $A_{2}$ and $A_{3}$.

a. Let $\lambda=\left(\lambda_{1} \geq \lambda_{2} \geq 0\right)$ and $\mu=\left(l^{3}\right)$ be partitions, $|\lambda|=|\mu|$.

Show that

$$
G_{\lambda}^{(3)}(q)=K_{\lambda,\left(l^{3}\right)}(q)=q^{c}\left[\begin{array}{c}
\min \left(\lambda_{1}-\lambda_{2}, \lambda_{2}\right)+1 \\
1
\end{array}\right]_{q},
$$

where $c=\max \left(\lambda_{1}-\lambda_{2}, \lambda_{2}\right)$.

b. Let $\lambda=\left(\lambda_{1} \geq \lambda_{2} \geq \lambda_{3} \geq 0\right)$ and $\mu=\left(l^{4}\right)$ be partitions, $|\lambda|=|\mu|$. Show that

$$
\begin{aligned}
& q^{2 l(l-1)-n(\lambda)} G_{\lambda}^{(4)}\left(q^{-1}\right)=\widetilde{K}_{\lambda,\left(l^{4}\right)}(q)=\left[\begin{array}{c}
\min \left(\lambda_{1}-\lambda_{2}, \lambda_{3}\right)+1 \\
1
\end{array}\right]_{q} \\
& \times \sum_{0 \leq a<\frac{\lambda_{2}-\lambda_{3}}{2}} q^{2 a}\left[\begin{array}{c}
\max \left(\lambda_{1}-\lambda_{2}, \lambda_{3}\right)+4 \min \left(l-\lambda_{3}-a, \lambda_{2}-l-a, 0\right)+1 \\
1
\end{array}\right]_{q} \\
& +\epsilon q^{\lambda_{2}-\lambda_{3}}\left[\begin{array}{c}
\min \left(\lambda_{1}-\lambda_{2}, \lambda_{3}\right)+2 \\
2
\end{array}\right]_{q},
\end{aligned}
$$


where $\epsilon=\frac{1+(-1)^{\lambda_{2}-\lambda_{3}}}{2}$.

c. Show that if $\lambda=\left(\lambda_{1} \geq \lambda_{2} \geq \cdots \geq \lambda_{N} \geq 0\right)$ is a partition such that $\lambda_{N} \geq l$, and $|\lambda|=(N+l) l$, then

$$
G_{\lambda}^{(N+1)}(q)=q^{n(\alpha)+l}\left[\begin{array}{l}
N \\
\alpha^{\prime}
\end{array}\right]
$$

where $\alpha=\left(\lambda_{1}-l, \lambda_{2}-l, \ldots, \lambda_{N}-l\right)$.

d. Let $\lambda=\left(\lambda_{1} \geq \cdots \geq \lambda_{N} \geq 0\right)$ be partition such that $l \geq \lambda_{2}$, and $|\lambda|=(N+1) l$. Show that

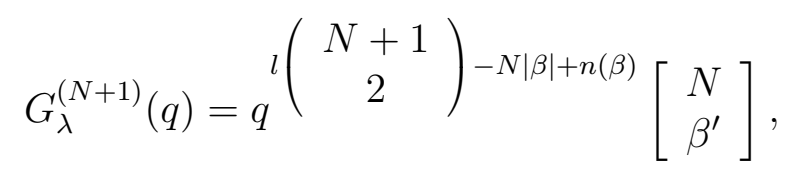

where $\beta=\left(\lambda_{2}, \lambda_{3}, \ldots, \lambda_{N}\right)$.

e. Let $\lambda=\left(\lambda_{1} \geq \lambda_{2} \geq \lambda_{3} \geq 0\right)$ be partition such that $\lambda_{1}=\lambda_{2}+\lambda_{3}$, and $|\lambda|=4 l$. Show that

$$
\widetilde{K}_{\lambda,\left(l^{4}\right)}(q)=\frac{\left(1-q^{\lambda_{2}-\lambda_{3}+1}\right)\left(1-q^{\lambda_{3}+1}\right)^{2}}{(1-q)^{2}\left(1-q^{2}\right)}+\epsilon q^{\lambda_{2}+1} \frac{1-q^{\lambda_{3}+1}}{1-q^{2}},
$$

where $\epsilon=\frac{1+(-1)^{\lambda_{1}}}{2}$.

\section{Internal product of Schur functions}

The irreducible characters $\chi^{\lambda}$ of the symmetric group $S_{n}$ are indexed in a natural way by partitions $\lambda$ of $n$. If $w \in S_{n}$, then define $\rho(w)$ to be the partition of $n$ whose parts are the cycle lengths of $w$. For any partition $\lambda$ of $m$ of length $l$, define the power-sum symmetric function

$$
p_{\lambda}=p_{\lambda_{1}} \ldots p_{\lambda_{l}}
$$

where $p_{n}(x)=\sum x_{i}^{n}$. For brevity write $p_{w}:=p_{\rho(w)}$. The Schur functions $s_{\lambda}$ and power-sums $p_{\mu}$ are related by a famous result of Frobenius

$$
s_{\lambda}=\frac{1}{n !} \sum_{w \in S_{n}} \chi^{\lambda}(w) p_{w}
$$

For a pair of partitions $\alpha$ and $\beta,|\alpha|=|\beta|=n$, let us define the internal product $s_{\alpha} * s_{\beta}$ of Schur functions $s_{\alpha}$ and $s_{\beta}$ :

$$
s_{\alpha} * s_{\beta}=\frac{1}{n !} \sum_{w \in S_{n}} \chi^{\alpha}(w) \chi^{\beta}(w) p_{w}
$$

It is well-known that

$$
s_{\alpha} * s_{(n)}=s_{\alpha}, \quad s_{\alpha} * s_{\left(1^{n}\right)}=s_{\alpha^{\prime}},
$$

where $\alpha^{\prime}$ denotes the conjugate partition to $\alpha$. 
Let $\alpha, \beta, \gamma$ be partitions of a natural number $n \geq 1$, consider the following numbers

$$
g_{\alpha \beta \gamma}=\frac{1}{n !} \sum_{w \in S_{n}} \chi^{\alpha}(w) \chi^{\beta}(w) \chi^{\gamma}(w) .
$$

The numbers $g_{\alpha \beta \gamma}$ coincide with the structural constants for multiplication of the characters $\chi^{\alpha}$ of the symmetric group $S_{n}$ :

$$
\chi^{\alpha} \chi^{\beta}=\sum_{\gamma} g_{\alpha \beta \gamma} \chi^{\gamma} .
$$

Hence, $g_{\alpha \beta \gamma}$ are non-negative integers. It is clear that

$$
s_{\alpha} * s_{\beta}=\sum_{\gamma} g_{\alpha \beta \gamma} s_{\gamma} .
$$

Let us introduce polynomials $L_{\alpha \beta}^{\mu}(q)$ via the decomposition of the internal product of Schur functions $s_{\alpha} * s_{\beta}(x)$ in terms of Hall-Littlewood functions:

$$
s_{\alpha} * s_{\beta}(x)=\sum_{\mu} L_{\alpha \beta}^{\mu}(q) P_{\mu}(x ; q) .
$$

It follows from (2.1) and (6.5) that

$$
L_{\alpha \beta}^{\mu}(q)=\sum_{\gamma} g_{\alpha \beta \gamma} K_{\gamma \mu}(q) .
$$

Thus, the polynomials $L_{\alpha \beta}^{\mu}(q)$ have non-negative integer coefficients, and

$$
L_{\alpha \beta}^{\mu}(0)=g_{\alpha \beta \mu} .
$$

The polynomials $L_{\alpha \beta}^{\mu}(q)$ can be considered as a generalization of the Kostka-Foulkes polynomials. Indeed, if partition $\beta$ consists of one part, $\beta=(n)$, then

$$
L_{\alpha \beta}^{\mu}(q)=K_{\alpha, \mu}(q) .
$$

Example 6.1 Take partitions $\alpha=(4,2)$ and $\beta=(3,2,1)$, then

$$
s_{\alpha} * s_{\beta}=s_{51}+2 s_{42}+2 s_{41^{2}}+s_{3^{2}}+3 s_{321}+2 s_{31^{3}}+s_{2^{3}}+2 s_{2^{2} 1^{2}}+s_{21^{4}} .
$$

Therefore,

$$
\begin{aligned}
& L_{\alpha \beta}^{(51)}(q)=1, \quad L_{\alpha \beta}^{(42)}(q)=(21), \quad L_{\alpha \beta}^{(411)}(q)=(231), \quad L_{\alpha \beta}^{(33)}(q)=(121), \\
& L_{\alpha \beta}^{(321)}(q)=(3531), \quad L_{\alpha \beta}^{\left(31^{3}\right)}(q)=(257531), \\
& \begin{aligned}
L_{\alpha \beta}^{\left(2^{3}\right)}(q) & =(135531), \quad L_{\alpha \beta}^{\left(2^{2} 1^{2}\right)}=(2699631), \\
L_{\alpha \beta}^{\left(21^{4}\right)}(q) & =(1,4,8,12,14,13,10,6,3,1) \\
& =(1+q)^{2}\left(1+q^{2}\right)\left(1+q+q^{2}\right)\left(1+q+q^{3}\right), \\
L_{\alpha \beta}^{\left(1^{6}\right)}(q) & =q(1,3,6,10,14,18,20,20,18,14,10,6,3,1) \\
& =q(1+q)^{3}\left(1+q^{2}\right)\left(1+q^{2}+q^{4}\right)^{2} .
\end{aligned}
\end{aligned}
$$


The value of polynomial $L_{\alpha \beta}^{\mu}(q)$ at $q=1$ admits the following combinatorial interpretation. First of all, for any skew shapes $A$ and $B$ consider the set $\boldsymbol{\nu}(A, B)$ of all $B$-tableaux of shape $A$. Let $b_{1}, b_{2}, \ldots$ be the row lengths of skew shape $B$, recall that a semistandard Young tableau $T$ of (skew) shape $A$ and weight $\left(b_{1}, b_{2}, \ldots\right)$ is called $B$-tableau, if the word $w(T)$ associated to $T$ (and given by reading the numbers in $T$ from right to left in successive rows, starting with the top row) is a $B$-lattice one.

Recall that for a given skew shape $B=\lambda \backslash \mu$, a word $w=w_{1} w_{2} \cdots w_{p}, w_{i} \in \mathbb{Z}_{>0}$, is called $B$-lattice, if for any $j, 1 \leq j \leq p$, such that $w_{j}=k>1$, the following inequality holds

$$
\begin{gathered}
\#\left\{i \mid i<j \text { and } w_{i}=w_{j}-1\right\}+\min \left(\lambda_{k}-\mu_{k}, \mu_{k-1}-\mu_{k}\right) \\
\geq \#\left\{i \mid i \leq j \text { and } w_{i}=w_{j}\right\} .
\end{gathered}
$$

In particular, if $B$ is a diagram (i.e. $\mu=0$ ) then the definitions (6.8) and (2.13) of $B$-lattice words and lattice words coincide.

Summarizing,

$$
\boldsymbol{\nu}(A, B)=\left\{T \in S T Y\left(A,\left(b_{1}, b_{2}, \ldots\right)\right) \mid w(T) \text { is a } B \text {-lattice word }\right\} .
$$

Let $\nu(A, B)$ be the number of $B$-tableaux of shape $A$. It follows from [9] and [73 that

$$
\nu(A, B)=\left\langle s_{A}, s_{B}\right\rangle=\operatorname{dim}_{\mathfrak{g l} l(n)}\left(V_{A}, V_{B}\right) .
$$

Note that if $A=\lambda \backslash \mu$ and $B$ is a diagram, say $B=\eta$, then $\nu(A, B)$ is equal to the Littlewood-Richardson number $c_{\mu \eta}^{\lambda}$, i.e. the number of semistandard Young tableaux $T$ of the (skew) shape $A$ and weight $\eta$ such that the corresponding word $w(T)$ is a lattice word.

Example 6.2 Take $A=(4332) \backslash(211), B=(5321) \backslash(21)$. Then

$$
K_{A,(3221)}(q)=\sum_{\pi} c_{211, \pi}^{4332} K_{\pi, 3221}(q)=\sum_{T \in S T Y(A,(3221))} q^{c(T)}=2+4 q+3 q^{2}+q^{3} .
$$

On the other hand, $\nu(A, B)=8$. It is easy to check that two semistandard Young tableaux $T_{1}, T_{2}$ of shape $A$ and weight (3221), which correspond to the words $w\left(T_{1}\right)=11214233$ and $w\left(T_{2}\right)=41213231$, do not belong to the set $\boldsymbol{\nu}(A, B)$.

Proposition 6.3 ([9]) Let $\alpha, \beta$ be partitions, $\mu$ be composition and $|\alpha|=|\beta|=|\mu|$. Then

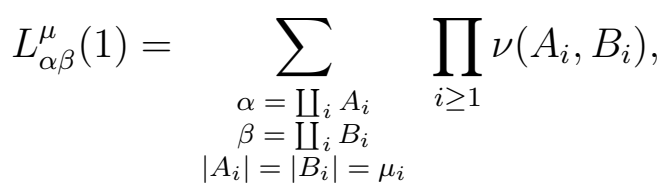

Here the symbol $\coprod_{i} A_{i}$ stands for the disjoint union of sets $A_{i}$. 
Definition 6.4 Let $\alpha, \beta$ be partitions, $\mu$ be composition, and $|\alpha|=|\beta|=|\mu|, l(\mu)=r$. A sequence of skew semistandard Young tableaux $\left(T_{1}, \ldots, T_{r}\right)$ is called Littlewood-Richardson sequence of type $(\alpha, \beta ; \mu)$, if there exist two sequences of partitions

$$
\left\{0=\alpha_{0} \subset \alpha_{1} \subset \alpha_{2} \subset \cdots \subset \alpha_{r}=\alpha\right\} \text { and }\left\{0=\beta_{0} \subset \beta_{1} \subset \cdots \subset \beta_{r}=\beta\right\},
$$

such that

i) $\left|\alpha_{i}\right|-\left|\alpha_{i-1}\right|=\left|\beta_{i}\right|-\left|\beta_{i-1}\right|=\mu_{i}, 1 \leq i \leq r$;

ii) $T_{i}$ is a $\left(\beta_{i} \backslash \beta_{i-1}\right)$-tableau of (skew) shape $\alpha_{i} \backslash \alpha_{i-1}, 1 \leq i \leq r$.

It is clear from (6.9) that $L_{\alpha \beta}^{\mu}(1)$ is equal to the number of Littlewood-Richardson sequences of tableaux $\left(T_{1}, \ldots, T_{r}\right)$ of type $(\alpha, \beta ; \mu)$. We will denote this set by $\boldsymbol{\nu}^{\mu}(\alpha, \beta)$ and its cardinality by $\nu^{\mu}(\alpha, \beta)$. From the very definition,

$$
L_{\alpha \beta}^{\mu}(1)=\nu^{\mu}(\alpha, \beta)=\sum_{\lambda} g_{\alpha \beta \lambda} K_{\lambda \mu} .
$$

It is not difficult to see that

$$
\nu^{\left(1^{n}\right)}(\alpha, \beta)=f^{\alpha} f^{\beta},
$$

where $f^{\alpha}$ denotes the number of standard Young tableaux of shape $\alpha$. For a $q$-analog of the latter formula, see Exercise 1 to this Section.

Problem 6.5 Find a statistics $d$ on the set $\boldsymbol{\nu}^{\mu}(\alpha, \beta)$ with the generating function $L_{\alpha \beta}^{\mu}(q)$.

Let $N \geq 2$, consider the principal specialization $x_{i}=q^{i}, 1 \leq i \leq N-1$, and $x_{i}=0$, if $i \geq N$, of the internal product of Schur functions $s_{\alpha}$ and $s_{\beta}$ :

$$
s_{\alpha} * s_{\beta}\left(q, q^{2}, \ldots, q^{N-1}\right)=\frac{1}{n !} \sum_{w \in S_{n}} \chi^{\alpha}(w) \chi^{\beta}(w) \prod_{k \geq 1}\left(\frac{q^{k}-q^{k N}}{1-q^{k}}\right)^{\rho_{k}(w)} .
$$

By a result of R.-K. Brylinski [4], Corollary 5.3, the polynomials

$$
s_{\alpha} * s_{\beta}\left(q, \ldots, q^{N-1}\right)
$$

admit the following interpretation. Let $P_{n, N}$ denote the variety of $n$ by $n$ complex matrices $z$ such that $z^{N}=0$. Denote by

$$
R_{n, N}:=\mathbb{C}\left[P_{n, N}\right]
$$

the coordinate ring of polynomial functions on $P_{n, N}$ with values in the field of complex numbers $\mathbb{C}$. This is a graded ring:

$$
R_{n, N}=\oplus_{k \geq 0} R_{n, N}^{(k)}
$$

where $R_{n, N}^{(k)}$ is a finite dimensional $\mathfrak{g} l(n)$-module with respect to the adjoint action. Let $\alpha$ and $\beta$ be partitions of common size. Then 4

$$
s_{\alpha} * s_{\beta}\left(q, \ldots, q^{N-1}\right)=\sum_{k \geq 0}\left\langle V_{[\alpha, \beta]_{n}}, P_{n, N}^{(k)}\right\rangle q^{k},
$$


as long as $n \geq \max (N l(\alpha), N l(\beta), l(\alpha)+l(\beta))$.

Our main result of this Section is Theorem 6.6 below, which connects the principal specialization of the internal product of Schur functions with certain parabolic Kostka polynomials, and gives, via Corollary 6.7, an effective method for computing the polynomials $s_{\alpha} * s_{\beta}\left(q, \ldots, q^{N-1}\right)$ which for the first time does not use the character table of the symmetric group $S_{n}$.

Theorem 6.6 i) Let $\alpha, \beta$ be partitions, $|\alpha|=|\beta|, l(\alpha) \leq r$, and $l(\alpha)+l(\beta) \leq N r$. Consider the sequence of rectangular shape partitions

$$
R_{N}=\{\underbrace{\left(\beta_{1}^{r}\right), \ldots,\left(\beta_{1}^{r}\right)}_{N}\} .
$$

Then

$$
s_{\alpha} * s_{\beta}\left(q, \ldots, q^{N-1}\right) \doteq K_{[\alpha, \beta]_{N r}, R_{N}}(q) .
$$

ii) (Dual form) Let $\alpha, \beta$ be partitions such that $|\alpha|=|\beta|, l(\alpha) \leq r$ and $l(\beta) \leq k$. For given non-negative integer $N$ such that $\alpha_{1}+\beta_{1} \leq N r$, consider partition

$$
\lambda_{N}:=\left(r N-\beta_{k}, r N-\beta_{k-1}, \ldots, r N-\beta_{1}, \alpha\right)
$$

and a sequence of rectangular shape partitions

$$
R_{N}:=(\underbrace{\left(r^{k}\right), \ldots,\left(r^{k}\right)}_{N}) .
$$

Then

$$
K_{\lambda_{N} R_{N}}(q) \doteq s_{\alpha} * s_{\beta}\left(q, \ldots, q^{N-1}\right)
$$

Corollary 6.7 (Fermionic formula for the principal specialization of the internal product of Schur functions, [31, 35]) Let $\alpha, \beta$ be partitions, $|\alpha|=|\beta|, l(\alpha)=r$. Then

$$
\begin{aligned}
& s_{\alpha} * s_{\beta}\left(q, \ldots, q^{N-1}\right) \\
& =\sum_{\nu} q^{c(\nu)} \prod_{k, j \geq 1}\left[\begin{array}{c}
P_{j}^{(k)}(\nu)+m_{j}\left(\nu^{(k)}\right)+N(k-1) \delta_{j, \beta_{1}} \theta(r-k) \\
P_{j}^{(k)}(\nu)
\end{array}\right]_{q},
\end{aligned}
$$

where the sum runs over all admissible configurations $\nu$ of type $\left([\alpha, \beta]_{N},\left(\beta_{1}^{N}\right)\right)$.

See Definitions 4.1 and 4.2 for explanation of symbols in the RHS(6.13).

Corollary 6.8 ([3]) Let $\alpha, \beta$ be partitions, $|\alpha|=|\beta|$. Then the principal specialization

$$
s_{\alpha} * s_{\beta}\left(q, \ldots, q^{N-1}\right)
$$

is a symmetric and unimodal polynomial. 
At the end of this Section we discuss a connection between the structural constants $g_{\alpha \beta \gamma}$ and the decomposition into irreducible parts of the exterior algebra

$$
\Lambda^{\bullet}(\mathfrak{g})=\oplus_{k \geq 0} \Lambda^{k}(\mathfrak{g})
$$

of the adjoint representation $\mathfrak{g}$ of the Lie algebra $\mathfrak{g} l(n)$.

To start, let us recall the well-known formula for the graded character $\operatorname{ch}_{q}\left(\Lambda^{\bullet}(\mathfrak{g})\right)$ of the exterior algebra of adjoint representation:

$$
\operatorname{ch}_{q}\left(\Lambda^{\bullet}(\mathfrak{g})\right)=\sum_{k \geq 0} q^{k} \operatorname{ch}\left(\Lambda^{k}(\mathfrak{g})\right)=\prod_{1 \leq i, j \leq n}\left(1+q x_{i} / x_{j}\right) .
$$

For any finite dimensional $\mathfrak{g} l(n)$-module $V$, let us consider the following polynomial

$$
E_{n}(V):=E_{n}(V ; q)=\sum_{k \geq 0} \operatorname{Mult}\left[V: \Lambda^{k}(\mathfrak{g})\right] q^{k} .
$$

Problem 6.9 Let $V_{\lambda}$ and $V_{\mu}$ be irreducible finite dimensional representations of the Lie algebra $\mathfrak{g l}(n)$. Compute the polynomials $E_{n}\left(V_{\lambda}\right)$ and $E_{n}\left(V_{\lambda} \otimes V_{\mu}^{*}\right)$.

By a theorem of Berenstein and Zelevinsky [2], it is known that

i) $\left.E_{n}\left(V_{\lambda}\right)\right|_{q=1}=2^{n} \operatorname{Mult}\left[V_{\lambda}: V_{\delta} \otimes V_{\delta}\right]$;

ii) ("Kostant conjecture")

- $E_{n}\left(V_{\lambda}\right) \neq 0$, if and only if $2 \delta \geq \lambda$ with respect to the dominance ordering on the set of partitions;

- $\left.E_{n}\left(V_{\lambda}\right)\right|_{q=1}=2^{n}$ if and only if there exists a permutation $w \in S_{n}$ such that $\lambda=\delta+w(\delta)$. Recall that $\delta:=\delta_{n}=(n-1, n-2, \ldots, 2,1,0)$.

Proposition $6.10\left(60\right.$, §4) Let $\lambda$ be partition, $2 \delta \geq \lambda$. Consider partition $\widetilde{\lambda}=\lambda+\left(1^{n}\right)$, then

$$
E_{n}\left(V_{\lambda}\right)=(1+q)\left\{\sum_{\mu=(a \mid b)} g_{n^{2}, \tilde{\lambda}, \mu} q^{b}\right\},
$$

summed over all hook partitions $\mu=\left(a+1,1^{b}\right), a+b=n^{2}-1$.

Proof. Consider the $q$-discriminant of order $n$ by $n$ :

$$
\Delta_{q}\left(X_{n}\right)=\prod_{1 \leq i, j \leq n}\left(x_{i}-q x_{j}\right) .
$$

It is a symmetric function in $X_{n}=\left(x_{1}, \ldots, x_{n}\right)$, and we can consider the decomposition of $q$-discriminant $\Delta_{q}\left(X_{n}\right)$ in terms of Schur functions $s_{\lambda}\left(X_{n}\right)$ :

$$
\Delta_{q}\left(X_{n}\right)=\sum_{\mu \vdash n^{2}} e r_{\mu}(q) s_{\mu}\left(X_{n}\right) .
$$


It is not difficult to see that

$$
E_{n}\left(V_{\lambda}\right)=e r_{\widetilde{\lambda}}(-q),
$$

where $\widetilde{\lambda}=\lambda+\left(1^{n}\right)$. On the other hand,

$$
\begin{aligned}
\Delta_{q}\left(X_{n}\right) & =s_{n^{2}}\left((1-q) X_{n}\right)=\sum_{\tilde{\lambda} \vdash n^{2}}\left(s_{n^{2}} * s_{\tilde{\lambda}}\right)(1-q) s_{\tilde{\lambda}}\left(X_{n}\right) \\
& =\sum_{\tilde{\lambda} \vdash n^{2}}\left(\sum_{\mu \vdash n^{2}} g_{n^{2}, \widetilde{\lambda}, \mu} s_{\mu}(1-q)\right) s_{\tilde{\lambda}}\left(X_{n}\right),
\end{aligned}
$$

so that

$$
\operatorname{er}_{\widetilde{\lambda}}(q)=\sum_{\mu \vdash n^{2}} g_{n^{2}, \widetilde{\lambda}, \mu} s_{\mu}(1-q) .
$$

It remains to use the well-known fact, see e.g. [52], that

$$
s_{\mu}(1-q)= \begin{cases}(1-q)(-q)^{b}, & \text { if } \mu=\left(a+1,1^{b}\right), \\ 0, & \text { otherwise }\end{cases}
$$

Thus, the problem of computation of polynomials $E_{n}\left(V_{\alpha}\right)$ is equivalent to finding the structure constants $g_{\alpha \beta \gamma}$ of the multiplication of the characters of the symmetric group $S_{n^{2}}$ in a particular case when $\alpha=\left(n^{2}\right), \beta \vdash n^{2}$ and $\gamma=(a \mid b)$ is a hook partition, $a+b=n^{2}-1$.

Proposition 6.11 Let $\alpha$ and $\beta$ be partitions of the same size $n^{2}-n$ whose length do not exceed $n$. Then

$$
E_{n}\left(V_{\alpha} \otimes V_{\beta}^{*}\right)=\sum_{\lambda \vdash n^{2}} K_{\lambda \widetilde{\alpha}}^{(-1)}(-q) K_{\lambda \widetilde{\beta}}^{(-1)}(-q) \prod_{i \geq 0}(-q ;-q)_{\lambda_{i}^{\prime}-\lambda_{i+1}^{\prime}},
$$

where $K_{\lambda \mu}^{(-1)}(q)$ denotes the so-called inverse Kostka-Foulkes polynomial, i.e. the $(\lambda, \mu)$-entry of the inverse Kostka-Foulkes matrix $\left(K_{\lambda, \mu}(q)\right)^{-1}$; in the product on the RHS(6.15) we made the convention that $\lambda_{0}^{\prime}=n$; the sum (6.13) is taken over partitions $\lambda$ of size $n^{2}$ such that $\lambda \leq(2 n+1,2 n-1, \ldots, 3,1)$ with respect to the dominance ordering on the set of partitions. Note that $K_{\lambda \mu}^{(-1)}(q)=0$, unless $\lambda \geq \mu$.

Formula (6.15) is a special case of a result obtained by M. Reeder 58]. Follow [58], we give below a proof of this formula which is based only on the theory of symmetric functions.

Proof of Proposition 6.11. Consider the ring $\Lambda_{n}(t)$ of symmetric polynomials in $n$ independent variables $x_{1}, \ldots, x_{n}$ with coefficients in the field $\mathbb{Q}(q)$ of rational functions in $q$. There exist two natural scalar products $($,$) and \langle$,$\rangle on the ring \Lambda_{n}(t)$ with values in the field $\mathbb{Q}(t)$. The first scalar product $($,$) is characterized uniquely by the condition that the$ set of all Schur functions

$$
\left\{s_{\lambda}\left(X_{n}\right), \quad \lambda \text { runs through all partitions of length } \leq n\right\}
$$


forms an orthonormal basis of $\Lambda_{n}(t)$ with respect to the scalar product ( , ). Similarly, the second scalar product $\langle$,$\rangle is defined uniquely by the condition that the set of all Hall-$ Littlewood polynomials

$$
\left\{P_{\lambda}\left(X_{n} ; q\right), \lambda \text { runs through all partitions of length } \leq n\right\}
$$

forms an orthogonal basis in $\Lambda_{n}(t)$ with respect to the scalar product

$$
\langle,\rangle:\left\langle P_{\lambda}, P_{\mu}\right\rangle=\frac{1}{w_{\lambda}(q)} \delta_{\lambda, \mu}
$$

where

$$
(1-q)^{n} w_{\lambda}(q)=b_{\lambda}(q)(q ; q)_{n-\lambda_{1}^{\prime}} .
$$

It is well-known, see e.g. [52, Chapter VI, $\S 9$, that these two scalar products are connected by the following relations:

$$
(f, g)=\left\langle f \Delta_{n}(q), g\right\rangle, \quad\langle f, g\rangle=\left(f / \Delta_{n}(q), g\right)
$$

where

$$
\Delta_{n}(q)=\prod_{1 \leq i \neq j \leq n}\left(1-q x_{i} / x_{j}\right)
$$

Now we are ready to prove Proposition 6.11. First of all, if

$$
s_{\beta}(x) \Delta_{n}(q)=\sum_{\mu} c_{\mu, \beta}(q) P_{\mu}(x ; q) w_{\mu}(q),
$$

then

$$
c_{\mu, \beta}(q)=\left\langle s_{\beta} \Delta_{n}(q), P_{\mu}\right\rangle=\left(s_{\beta}, P_{\mu}\right)
$$

Thus,

$$
\begin{aligned}
E_{n}\left(V_{\alpha} \otimes V_{\beta}^{*} ; q\right) & =\left(s_{\alpha}, s_{\beta} \Delta_{n}(q)\right)=\sum_{\mu} c_{\mu, \beta}(q) w_{\mu}(q)\left(s_{\alpha}, P_{\mu}\right) \\
& =\sum_{\mu}\left(s_{\alpha}, P_{\mu}\right)\left(s_{\beta}, P_{\mu}\right) w_{\mu}(q) .
\end{aligned}
$$

It remains to observe that since

$$
s_{\alpha}(x)=\sum_{\eta} K_{\alpha \eta}(q) P_{\eta}(x ; q)
$$

the scalar product $\left(s_{\alpha}, P_{\mu}\right)$ is equal to the inverse Kostka-Foulkes polynomial $K_{\mu \alpha}^{(-1)}(q)$. 
Remark 6.12 Similar arguments may be used to prove the statement iii) of Theorem 5.2. Namely,

$$
\begin{aligned}
G_{n}\left(V_{\alpha} \otimes V_{\beta}^{*}\right) & =\frac{(q ; q)_{n}}{(1-q)^{n}}\left(s_{\alpha}, s_{\beta} / \Delta_{n}(q)\right)=[n] !\left\langle s_{\alpha}, s_{\beta}\right\rangle \\
& =\sum_{\mu} \frac{K_{\alpha \mu}(q) K_{\beta \mu}(q)}{b_{\mu}(q)} \frac{(q ; q)_{n}}{(q ; q)_{n-\mu_{1}^{\prime}}}
\end{aligned}
$$

Remark 6.13 Combinatorial interpretation of the inverse Kostka-Foulkes polynomials $K_{\lambda \mu}^{(-1)}(q)$ was obtained by Egecioglu and Remmel [11]. See also an interesting paper by J.O. Carbonara in Discrete Math. 193 (1998), 117-145.

\section{Exercises}

1. Let $\alpha, \beta$ be partitions, $|\alpha|=|\beta|=n$. Show that

$$
L_{\alpha \beta}^{\left(1^{n}\right)}(q)=K_{\beta^{\prime} \alpha}(q, q) \widetilde{K}_{\alpha,\left(1^{n}\right)}(q)=K_{\alpha^{\prime} \beta}(q, q) \widetilde{K}_{\beta,\left(1^{n}\right)}(q)
$$

where

$$
K_{\alpha \beta}(q, q):=\left.K_{\alpha \beta}(q, t)\right|_{q=t},
$$

and $K_{\alpha \beta}(q, t)$ stands for the double Kostka polynomial introduced by I. Macdonald [52], Chapter VI, (8.11).

2. (Two variable generalization of polynomials $\left.L_{\alpha \beta}^{\mu}(q)\right)$ Let $\alpha, \beta, \mu$ be partitions, $|\alpha|=|\beta|=$ $|\mu|=n$, define

$$
L_{\alpha \beta}^{\mu}(q, t)=\sum_{\gamma} g_{\alpha \beta \gamma} K_{\gamma \mu}(q, t)
$$

Polynomials $L_{\alpha \beta}^{\mu}(q, t)$ may be considered as a generalization of the double Kostka polynomials $K_{\alpha \mu}(q, t)$. Indeed, if $\beta=(n)$, then

$$
L_{\alpha \beta}^{\mu}(q, t)=K_{\alpha \mu}(q, t), \text { and } L_{\alpha\left(1^{n}\right)}^{\mu}(q, t)=K_{\alpha^{\prime} \mu}(q, t) .
$$

Polynomials $L_{\alpha \beta}^{\mu}(q, t)$ have properties similar to those of $K_{\alpha \mu}(q, t)$.

Show that

i) $L_{\alpha \beta}^{\mu}(0, t)=L_{\alpha \beta}^{\mu}(t)$;

ii) $L_{\alpha \beta}^{\mu}(0,0)=g_{\alpha \beta \mu}, L_{\alpha \beta}^{\mu}(1,1)=f^{\alpha} f^{\beta}$, where $f^{\alpha}$ denotes the number of standard (i.e. weight $\left.\left(1^{|\alpha|}\right)\right)$ Young tableaux of shape $\alpha$;

iii) $L_{\alpha \beta}^{\mu}(q, t)=L_{\alpha^{\prime} \beta^{\prime}}^{\mu^{\prime}}(t, q)$;

iv) $L_{\alpha \beta}^{\mu}(q, t)=q^{n\left(\mu^{\prime}\right)} t^{n(\mu)} L_{\alpha^{\prime} \beta}^{\mu}\left(q^{-1}, t^{-1}\right)$;

v) $L_{\alpha \beta}^{1^{n}}(q, t)=K_{\alpha^{\prime} \beta}(t, t) \widetilde{K}_{\beta,\left(1^{n}\right)}(t)=K_{\beta^{\prime} \alpha}(t, t) \widetilde{K}_{\alpha,\left(1^{n}\right)}(t)$.

vi) let $\alpha, \beta$ and $\mu$ be partitions of the same size, then

$$
\sum_{\eta} K_{\mu \eta}(q) L_{\alpha \beta}^{\eta}(q) / b_{\eta}(q)=L_{\alpha \beta}^{\mu}(q, q) / H_{\mu}(q)
$$


where $H_{\mu}(q)$ denotes the hook polynomial $\prod_{x \in \mu}\left(1-q^{h(x)}\right)$, and for any partition $\eta$ we put $b_{\eta}(q)=\prod_{i \geq 1}(q ; q)_{\eta_{i}^{\prime}-\eta_{i+1}^{\prime}}$, c.f. Corollary 5.6.

3. Let $\lambda$ and $\mu$ be partitions and $\lambda \geq \mu$ with respect to the dominance ordering on the set of partitions. Denote by $d(\alpha \beta \mu)$ the degree of polynomial $L_{\alpha \beta}^{\mu}(q)$, and consider polynomial

$$
\widetilde{L}_{\alpha \beta}^{\mu}(q)=q^{d(\alpha \beta \mu)} L_{\alpha \beta}^{\mu}\left(q^{-1}\right)
$$

Show that

$$
\widetilde{L}_{\alpha \beta}^{\mu}(q) \geq \widetilde{L}_{\alpha \beta}^{\lambda}(q)
$$

i.e. the difference $\widetilde{L}_{\alpha \beta}^{\mu}(q)-\widetilde{L}_{\alpha \beta}^{\lambda}(q)$ is a polynomial with non-negative coefficients. More generally, let $I:=\left[\mu_{1}, \mu_{2}\right]$ be an interval in the Young graph $(\mathbb{Y}, \leq)$, then

$$
\sum_{\tau \in\left[\mu_{1}, \mu_{2}\right]} \mu(\tau ; I) \widetilde{L}_{\alpha \beta}^{\tau}(q) \geq 0
$$

where $\mu(\tau ; I)$ denotes the Möbius function of the interval $I$.

If partition $\alpha$ (or $\beta$ ) consists of only one part, the latter inequality was discovered and proved by Lascoux and Schützenberger [45]; see also [30] for yet another proof.

- Construct a natural embedding of the sets

$$
\boldsymbol{\nu}^{\mu}(\alpha, \beta) \hookrightarrow \boldsymbol{\nu}^{\lambda}(\alpha, \beta)
$$

Hence, for any partition $\mu$ there exists a natural embedding (standardization map)

$$
i_{\mu}: \boldsymbol{\nu}^{\mu}(\alpha, \beta) \hookrightarrow S T Y(\alpha) \times S T Y(\beta)
$$

Problem 6.14 Describe the image of the standardization map (6.10).

Problem 6.15 Let $\alpha$ and $\beta$ be partitions, define coefficients $r_{\alpha \beta k}^{(N)}$ via the decomposition

$$
s_{\alpha} * s_{\beta}\left(q, \ldots, q^{N-1}\right)=\sum_{k=0}^{N|\alpha|} r_{\alpha \beta k}^{(N)} q^{k}
$$

It follows from Corollary 6.8 that if $1 \leq k \leq \frac{1}{2} N|\alpha|$, then

$$
g_{\alpha \beta k}^{(N)}:=r_{\alpha \beta, k}^{(N)}-r_{\alpha \beta, k-1}^{(N)} \geq 0 .
$$

Give a combinatorial, algebra-geometrical and representation theoretical interpretations of the non-negative integer numbers $g_{\alpha \beta k}^{(N)}$. 
4. Let $N \geq 1, M \geq 1$ be integer numbers. For any pair of partitions $\alpha$ and $\beta$ of the same size $n$, consider the following polynomial (cf. (6.10)):

$$
\begin{aligned}
& S_{\alpha \beta ; N, M}(q)= \\
& \frac{1}{n !} \sum_{w \in S_{n}} \chi^{\alpha}(w) \chi^{\beta}(w) \prod_{k \geq 1}\left(\frac{q^{k}-\left(1+(-1)^{k}\right) q^{k N}+(-1)^{k} q^{k(N+M-1)}}{1-q^{k}}\right)^{\rho_{k}(w)} .
\end{aligned}
$$

It is clear that

$$
\begin{aligned}
S_{\alpha \beta ; N, 1}(q) & =s_{\alpha} * s_{\beta}\left(q, \ldots, q^{N-1}\right) \\
S_{\alpha \beta ; 2,2}(q) & =(1+q) \sum_{\mu=(a \mid b)} g_{\alpha \beta \mu} q^{b}
\end{aligned}
$$

summed over all hook partitions $\mu=\left(a+1,1^{b}\right), a+b=n-1$.

- Show that $S_{\alpha \beta ; N, M}(q)$ is a polynomial with non-negative integer coefficients.

Example 6.16 Take partitions $\alpha=(31)$ and $\beta=(22)$. Using the character table for the symmetric group $S_{4}$, one can easily find the following expression for the internal product of Schur functions in question:

$$
s_{\alpha} * s_{\beta}=\frac{1}{4 !}\left(6 p_{1}^{4}-6 p_{2}^{2}\right)
$$

and therefore,

$$
\begin{aligned}
S_{\alpha \beta ; n, m}(q) & =q^{5}\left[\begin{array}{c}
n+m-2 \\
1
\end{array}\right]_{q}\left[\begin{array}{c}
2 n+2 m-5 \\
1
\end{array}\right]_{q}+q^{7}\left[\begin{array}{l}
3 \\
1
\end{array}\right]_{q}\left[\begin{array}{c}
n+m-1 \\
4
\end{array}\right]_{q} \\
& +q^{9}\left[\begin{array}{l}
3 \\
1
\end{array}\right]_{q}\left[\begin{array}{c}
n+m-2 \\
4
\end{array}\right]_{q}+q^{2 n+2}\left[\begin{array}{c}
n-1 \\
1
\end{array}\right]_{q^{2}}\left[\begin{array}{c}
m-1 \\
1
\end{array}\right]_{q^{2}} .
\end{aligned}
$$

In particular,

$$
S_{\alpha \beta ; 2,2}(q)=q^{5}\left(1+q+q^{2}\right)+q^{6}=q^{5}(1+q)^{2} .
$$

Problem 6.17 Find a fermionic formula for polynomials $S_{\alpha \beta ; N, M}(q)$ which generalizes that 6.13)

5. Let $\alpha$ be partition of size $n \geq 2$. Consider the new partition

$$
\lambda_{\alpha}=\alpha+\left((n-2)^{n}\right)=\left(\alpha_{1}+n-2, \alpha_{2}+n-2, \ldots, \alpha_{n}+n-2\right) .
$$

Show that

$$
E_{n}\left(V_{\lambda_{\alpha}}\right)=\prod_{(i, j) \in \alpha}\left(1+q^{2(i-j)+1}\right) K_{\alpha,\left(1^{n}\right)}\left(q^{2}\right) .
$$


In particular, if $\alpha=\left(1^{n}\right)$, then

$$
E_{n}\left(\lambda_{\alpha}\right)=\prod_{j=1}^{n}\left(1+q^{2 j-1}\right)
$$

is the Poincare polynomial of the unitary group $U(n)$; if $\alpha=\left(2,1^{n-2}\right)$, i.e. the highest weight of the adjoint representation, then

$$
E_{n}\left(V_{\lambda_{\alpha}}\right)=\prod_{j=1}^{n-1}\left(1+q^{2 j-1}\right) \frac{1-q^{2(n-1)}}{1-q} .
$$

Formula (6.18) was stated as a conjecture by Gupta and Hanlon [22] and has been proved by Stembridge [68.

6. Assume that partition $\lambda$ has the form $\lambda=\delta_{n}+w\left(\delta_{n}\right)$ for some permutation $w \in S_{n}$. Show that

$$
\left.E_{n}\left(V_{\lambda}\right)=q^{l\left(w w_{0}\right.}\right) \prod_{k \geq 1}\left(1+q^{2 k-1}\right)^{a_{k}(w)-a_{k-1}(w)},
$$

where $a_{k}(w)$ is equal to the length of the longest subsequence of $w$ that can be written as union of $k$ increasing subsequences.

7. (Explicit description of partitions $\lambda$ such that $\left.E_{n}\left(V_{\lambda}\right)\right|_{q=1}=2^{n}$ )

By a theorem of B. Kostant (see [2], Proposition 13) and that of A. Berenstein and A. Zelevinsky [2], Theorem 14, the partitions $\lambda$ such that $\left.E_{n}\left(V_{\lambda}\right)\right|_{q=1}=2^{n}$ are in a bijective correspondence with subsets $I \subset[1, n-1]$ : partition $\lambda(I)$ corresponding to $I$ is equal to $\delta_{n}+w_{I}\left(\delta_{n}\right)$, where $w_{I}$ is the element of the maximal length in the Weyl group generated by the simple reflections $s_{i}, i \in I$. The main purpose of the Exercises 6 and 7 is to give a direct construction of the partition $\lambda(I)$, and describe explicitly the RHS(6.19) in terms of the subset $I$.

Let $I$ be a subset of the interval $[1, n-1]$. For each $j \in[1, n] \backslash I$ we define two numbers $k_{I}^{+}(j)$ and $k_{I}^{-}(j)$ :

- Let us put $k_{I}^{+}(n-1)=k_{I}^{+}(n)=0$, and if $1 \leq j \leq n-2$, then

$$
k_{I}^{+}(j)=\max \{k \mid[j+1, \ldots, j+k] \subset I\} .
$$

Thus, we have $k_{I}^{+}(j)=0$, if $j+1 \notin I$.

- Let us put $k_{I}^{-}(1)=0$, and if $2 \leq j \leq n$, then

$$
k_{I}^{-}(j)=\max \{k \mid[j-k, \ldots, j-1] \subset I\} .
$$

Thus, we have $k_{I}^{-}(j)=0$, if $j-1 \notin I$.

Finally, let us define partition $\lambda(I)=\left(\lambda_{1}(I), \ldots, \lambda_{n}(I)\right)$ by the rule

$$
\lambda(I)_{i}=\sum_{j \geq i}\left(2+k_{I}^{-}(j)+k_{I}^{+}(j)\right) \chi(j \notin I) .
$$

- Show that 


$$
\left.E\left(V_{\lambda}\right)\right|_{q=1}=2^{n}
$$

if and only if there exists a subset $I \subset[1, n-1]$, such that $\lambda=\lambda(I)$. Thus, the number of partitions $\lambda$ such that $\left.E\left(V_{\lambda}\right)\right|_{q=1}=2^{n}$ is equal to $2^{n-1}$.

8. Let $I=\left\{1 \leq j_{1}<j_{2} \leq \cdots<j_{p} \leq n-1\right\}$ be a subset of the interval [1,n-1]. Define by induction the composition $m=\left(m_{1}, \ldots, m_{s}\right)$ of the number $p=\#|I|$. First of all, put $m_{0}=1$. If $i \geq 1$ and the numbers $m_{0}, \ldots, m_{i-1}$ have been already defined, then put $m_{i}=$

$$
\max \left\{k \in\left[1, p+1-m_{0}-\cdots-m_{i-1}\right] \mid j_{m_{0}+\cdots+m_{i-1}+k-1}=j_{m_{0}+\cdots+m_{i-1}}+k-1\right\} \text {. }
$$

Let $s:=\max \left\{j \mid m_{j} \neq 0\right\}$, it is clear that $m_{1}+\cdots+m_{s}=p$.

Show that

$$
E_{n}\left(V_{\lambda(I)}\right)=q^{c(I)}(1+q)^{n-p} \prod_{j=1}^{s} \prod_{r=1}^{m_{j}}\left(1+q^{2 r+1}\right)
$$

where $c(I)=\left(\begin{array}{c}n \\ 2\end{array}\right)-\sum_{j=1}^{s}\left(\begin{array}{c}m_{j}+1 \\ 2\end{array}\right)$.

In particular, if $I=\{1,2, \ldots, \widehat{j}, \ldots, n-1\}$, then

$$
E_{n}\left(V_{\lambda(I)}\right)=q^{j(n-j)}(1+q)^{2} \prod_{r=1}^{j-1}\left(1+q^{2 r+1}\right) \prod_{r=1}^{n-j-1}\left(1+q^{2 r+1}\right) .
$$

9. Let $\lambda=\left(\lambda_{1}, \lambda_{2}, \ldots, \lambda_{n}\right)$ be a partition. Show that

- (Reduction formulas)

i) if $\lambda \geq 2 \delta_{n+1}$, then $E_{n+1}\left(V_{\lambda}\right)=q^{n}(1+q) E_{n}\left(V_{\widetilde{\lambda}}\right)$,

where $\widetilde{\lambda}=\left(\lambda_{1}-2, \ldots, \lambda_{n}-2\right)$;

ii) if $\lambda \geq 2 \delta_{n}$, then $E_{n+1}\left(V_{\widehat{\lambda}}\right)=q^{n}(1+q) E_{n}\left(V_{\lambda}\right)$,

where $\widehat{\lambda}=\left(2 n, \lambda_{1}, \ldots, \lambda_{n}\right)$.

- (Duality symmetry)

$E_{n}\left(V_{\lambda}\right)=E_{n}\left(V_{\lambda}^{*}\right)=E_{n}\left(V_{\lambda^{+}}\right)$,

where $\lambda^{+}=\left(2 n-2-\lambda_{n}, 2 n-2-\lambda_{n-1}, \ldots, 2 n-2-\lambda_{1}\right)$.

In the case $q=1$ these formulae may be found in [60, $§ \S 5,6$.

- (Poincare duality)

$E_{n}(V ; q)=q^{n(n-1)} E_{n}\left(V ; q^{-1}\right)$.

10. Show that

$$
\sum_{\lambda \leq 2 \delta_{n}} E_{n}\left(V_{\lambda} ;-q\right) G_{n}\left(V_{\lambda} ; q\right)=1
$$

where $G_{n}(V ; q)$ is the generalized exponents polynomial, see (5.1).

11. Let $\lambda=\left(\lambda_{1} \geq \lambda_{2} \geq \cdots \geq \lambda_{k}>0\right)$ be a partition of $n$. Show that

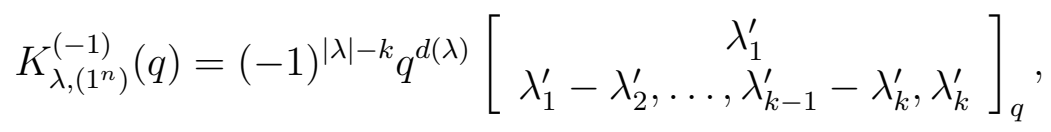


where $d(\lambda)=n(\lambda)+|\lambda|+k(k+1) / 2$.

Formula (6.20) was conjectured by J.O. Carbonara and proved by I.G. Macdonald, see Discr. Math. 193 (1998), 117-145, Proposition 3.

12. Let $\alpha$ and $\beta$ be partitions of the same size $n$. Show that

$$
s_{\alpha} * s_{\beta}(1-q)=\sum_{\mu} K_{\mu \alpha}^{(-1)}(q) K_{\mu \beta}^{(-1)}(q) b_{\mu}(q)=H_{\beta}(q) K_{\beta \alpha}^{(-1)}(q, q) \text {, }
$$

where $K_{\beta \alpha}^{(-1)}(q, q)$ denotes the $(\beta, \alpha)$-entry of the inverse matrix $\left(\left(K_{\lambda \mu}(q, q)\right)_{\lambda, \mu \vdash n}\right)^{-1}$ of size $p(n)$ by $p(n)$. Here $p(n)$ stands for the number of partitions of size $n$. Recall, see [52], Chapter VI, Example 6, p.364, that

$$
\operatorname{det}\left|\left(K_{\lambda \mu}(q, q)\right)_{\lambda, \mu \vdash n}\right|=\prod_{\lambda \vdash n} \frac{H_{\lambda}(q)}{b_{\lambda}(q)} .
$$

13. (Generalization of Corollary 6.8) Let $\alpha$ and $\beta$ be partitions of the same size and $\left(k_{1}, k_{2}, \ldots, k_{r}\right)$ be a unimodal and symmetric sequence of non-negative integer numbers.

Show that the specialization

$$
s_{\alpha} * s_{\beta}(\underbrace{q, \ldots, q}_{k_{1}}, \underbrace{q^{2}, \ldots, q^{2}}_{k_{2}}, \ldots, \underbrace{q^{r}, \ldots, q^{r}}_{k_{r}})
$$

is a symmetric and unimodal polynomial.

This result is due essentially to A. Kerber, see e.g., R. Stanley (Studies in App. Math. 72 (1985), 263-281), or 65], Theorem 14.

Question. Does there exist a fermionic formula for polynomials (6.21), which generalizes that $(6.13)$ ?

14. Let $\lambda$ be a partition of size $n$. Consider partition $\widetilde{\lambda}=(3 n, 2 n, \lambda)$ and a sequence of rectangular shape partitions $R:=((n, n),(n, n),(n),(n))$. Let $s_{\lambda}\left(1, q, q, q^{2}\right)$ denotes the specialization $x_{1}=1, \quad x_{2}=x_{3}=q, \quad x_{4}=q^{2}, x_{k}=0$, if $k \geq 5$, of the Schur function $s_{\lambda}(x)$. Show that

$$
s_{\lambda}\left(1, q, q, q^{2}\right) \doteq K_{\widetilde{\lambda} R}(q)
$$

\section{$7 \quad$ Liskova semigroup}

\subsection{Realizable polynomials and product formula}

Definition 7.1 A polynomial $P(q) \in \mathbb{N}[q]$ with non-zero constant term is called $K$-realizable if there exist two partitions $\lambda$ and $\mu,|\lambda|=|\mu|$, a composition $\eta,|\eta|=l(\mu)$, and an integer $L$ such that

$$
P(q)=q^{L} K_{\lambda \mu \eta}(q)
$$

The set of all $K$-realizable polynomials will be denoted by $\mathfrak{L}$. 
Definition 7.2 A polynomial $P(q) \in \mathbb{N}[q]$ with non-zero constant term is called $P K$-realizable if there exist a partition $\lambda$, a dominant sequence of rectangular shape partitions $R$, and an integer $L$ such that

$$
P(q)=q^{L} K_{\lambda R}(q)
$$

The set of all PK-realizable polynomials will be denoted by $\mathfrak{L}^{+}$.

Definition 7.3 A polynomial $P(q) \in 1+q \mathbb{N}[q]$ is called $K F$-realizable, if there exist partitions $\lambda$ and $\mu$ such that

$$
P(q)=\widetilde{K}_{\lambda \mu}(q)
$$

The set of all KF-realizable polynomials will be denoted by $\mathfrak{L}^{++}$. It is clear that $\mathfrak{L}^{++} \subset$ $\mathfrak{L}^{+} \subset \mathfrak{L}$.

Problem 7.4 Describe the sets $\mathfrak{L}, \mathfrak{L}^{+}$and $\mathfrak{L}^{++}$.

Conjecture 7.5 If $n \geq 3$ and $a \in \mathbb{Z}_{\geq 1}$, then $1+a q^{n} \notin \mathfrak{L}$.

Conjecture 7.6 Let $K_{\lambda R}(q)=q^{\bullet}\left(a_{0}, a_{1}, \ldots, a_{l}\right)$ be parabolic Kostka polynomial corresponding to a partition $\lambda$ and a dominant sequence of rectangular shape partitions $R$. Then both sequences $\left(a_{0}, a_{2}, a_{4}, \ldots\right)$ and $\left(a_{1}, a_{3}, a_{5}, \ldots\right)$ are unimodal.

What one can say about the structure of the sets $\mathfrak{L} \mathfrak{L}^{+}$, and $\mathfrak{L}^{++}$? First of all, it follows from Duality Theorem that if a polynomial $P(q)$ belongs to the set $\mathfrak{L}^{+}$, then the polynomial $q^{\operatorname{deg}(P)} P\left(q^{-1}\right)$ also belongs to the set $\mathfrak{L}^{+}$. Another interesting property of the set $\mathfrak{L}^{+}$follows from the following result.

Theorem 7.7 Let $\alpha$ and $\beta$ be partitions, and $R, S=\left\{\left(\mu_{a}^{\eta_{a}}\right)\right\}, 1 \leq a \leq p$, be two dominant sequences of rectangular shape partitions. Let $k=\sum \mu_{a}$, and $n$ be such that $n \geq l(\alpha)+l(\beta)$, and $n \geq \eta_{a}, 1 \leq a \leq p$. Consider partition

$$
[\alpha, \beta]_{n, k}:=(\alpha_{1}+k, \alpha_{2}+k, \ldots, \alpha_{s}+k, \underbrace{k, \ldots k}_{n-s-r}, k-\beta_{r}, \ldots, k-\beta_{1}),
$$

and a dominant rearrangement $Q$ of the sequence of rectangular shape partitions $R \cup\left\{\left(\mu_{a}^{n-\eta_{a}}\right)\right\}_{a=1}^{p}$. Then

$$
K_{[\alpha, \beta]_{n, k} Q}(q)=K_{\alpha R}(q) K_{\beta S}(q) .
$$

It follows from Theorem 7.7 that the set of PK-realizable polynomials $\mathfrak{L}^{+}$is closed under multiplication, and forms a semigroup called by Liskova semigroup.

Question. Denote by $\mathfrak{K}_{n}^{+}$the set of all degree $n$ parabolic Kostka polynomials $K_{\lambda R}(q)$ corresponding to a partition $\lambda$ and a dominant sequence of rectangular shape partitions $R$. Is it true that the set $\mathfrak{K}_{n}^{+}$is finite for all $n \geq 0$ ? If so, what does the generating function $\sum_{n \geq 0}\left|\mathfrak{K}_{n}^{+}\right| t^{n}$ looks like? 
Conjecture 7.8 Let $P(q)$ be a polynomial with non-negative integer coefficients and nonzero constant term. There exists a non-negative integer $N$, depending on $P(q)$, such that the product $(1+q)^{N} P(q)$ belongs to the Liskova semigroup $\mathfrak{L}^{+}$.

The least non-negative integer $N$ such that $(1+q)^{N} P(q) \in \mathfrak{L}^{+}$will be denoted by $I L(P)$. For example, one can show that if $n$ and $m$ are non-negative integers then $I L(n) \leq 2$, see e.g., Section 4 , Exercise 6, and $I L(n+m q) \leq|n-m|+\delta_{n, m}$.

Problem 7.9 Given an integer $n \geq 3$, compute the number $I L\left(1+q^{n}\right)$.

\subsection{Generalized $q-$ Gaussian coefficients}

Let $\lambda$ be a partition, and

$$
\left[\begin{array}{l}
n \\
\lambda
\end{array}\right]:=\prod_{x \in \lambda} \frac{1-q^{c(x)}}{1-q^{h(x)}}
$$

denotes the generalized $q$-Gaussian coefficient, [52], Chapter I, §3, Example 1. Then the polynomial $\left[\begin{array}{l}n \\ \lambda\end{array}\right]$ is KF-realizable. This statement follows from [32], Lemma 1. Namely, let $\lambda$ be a partition, consider partitions

$$
\lambda_{N}=(N|\lambda|, \lambda):=\left(N|\lambda|, \lambda_{1}, \lambda_{2}, \ldots\right), \text { and } \mu_{N}=\left(|\lambda|^{N+1}\right)=(\underbrace{|\lambda|, \ldots,|\lambda|}_{N+1}) .
$$

Then

$$
\widetilde{K}_{\lambda_{N} \mu_{N}}(q)=\left[\begin{array}{l}
N \\
\lambda^{\prime}
\end{array}\right]
$$

See [32, 35] for proof and further details. It has been shown in 33, 35] that the identity (7.1) can be considered as a generalization of the $\mathrm{KOH}$ identity (4.5), see [56, 71, 32].

Identity (7.1) admits a generalization. To start, let us recall the well-known facts (see, e.g. [52], Chapter I) that

$$
s_{\lambda}\left(1, \ldots, q^{N-1}\right)=q^{n(\lambda)}\left[\begin{array}{c}
N \\
\lambda^{\prime}
\end{array}\right], \quad s_{\lambda} * s_{(n)}\left(1, \ldots, q^{N-1}\right)=s_{\lambda}\left(1, \ldots, q^{N-1}\right)
$$

and hence

$$
s_{\lambda} * s_{(n)}\left(1, \ldots, q^{N-1}\right) \stackrel{\bullet}{=} K_{\lambda_{N} \mu_{N}}(q),
$$

where $\lambda_{N}=(N|\lambda|, \lambda)$, and $\mu_{N}=\left(|\lambda|^{N+1}\right)$. We will give below a generalization of (7.3) when partition $(n)$ is replaced by an arbitrary rectangular shape partition.

Theorem 7.10 Let $\lambda$ be a partition, and $R=\left(l^{m}\right)$ be a rectangular shape partition, $|\lambda|=$ $l m$. Consider the partition $\lambda_{N}=(\underbrace{N l, \ldots N l}_{m}, \lambda)$ and the sequence $Q_{N}$ of rectangular shape partitions $Q_{N}=(\underbrace{R, \ldots, R}_{N+1})$. Then

$$
s_{\lambda} * s_{R}\left(q, \ldots, q^{N-1}\right) \doteq K_{\lambda_{N} Q_{N}}(q) .
$$


Remark 7.11 In Section 6, Theorem 6.6, we gave another generalization of the identity (7.3) when partition $(n)$ is replaced by arbitrary partition $\beta,|\beta|=n$. As a corollary, we obtain the following identity

$$
K_{[\alpha, R]_{N r}, R_{N}}(q) \doteq K_{\alpha_{N}, Q_{N}}(q),
$$

where

$$
\begin{aligned}
{[\alpha, R]_{N r} } & =(\alpha_{1}+l, \ldots, \alpha_{r}+l, \underbrace{l, \ldots, l}_{N r-r-m}), \\
\alpha_{N} & =(\underbrace{N l, \ldots, N l}_{m}, \alpha_{1}, \ldots, \alpha_{r}), \\
R_{N} & =\left\{\left(l^{r}\right)^{N}\right\}, \quad Q_{N}=\left\{\left(l^{m}\right)^{N+1}\right\},
\end{aligned}
$$

and $r=l(\alpha)$.

\subsection{Multinomial coefficients}

Lemma 7.12 ([52], Chapter I) Let $\lambda=\left(n, 1^{m}\right)$ be a hook, and $\mu$ be a partition. Then

$$
\widetilde{K}_{\lambda \mu}(q)=\left[\begin{array}{c}
\mu_{1}^{\prime}-1 \\
m
\end{array}\right]_{q} .
$$

It follows from Lemma 7.12 and Theorem 7.7 that the $q$-multinomial coefficient

$$
\left[\begin{array}{c}
N \\
m_{1}, \ldots, m_{k}
\end{array}\right]_{q}:=\frac{(q ; q)_{N}}{(q ; q)_{m_{1}} \cdots(q ; q)_{m_{k}}}
$$

where $m_{i}, 1 \leq i \leq k$, are non-negative integers and $N=m_{1}+\cdots+m_{k}$, belongs to the Liskova semigroup $\mathfrak{L}^{+}$. Indeed,

$$
\left[\begin{array}{c}
N \\
m_{1}, \ldots, m_{k}
\end{array}\right]_{q}=\left[\begin{array}{c}
N \\
m_{1}
\end{array}\right]_{q}\left[\begin{array}{c}
N-m_{1} \\
m_{2}
\end{array}\right]_{q} \cdots\left[\begin{array}{c}
N-m_{1}-\cdots-m_{k-1} \\
m_{k}
\end{array}\right]_{q} .
$$

Question. Is it true that the $q-$ multinomial coefficients belong to the set $\mathfrak{L}^{++}$?

Note, that according to Section [3, (3.23), the $q^{2}$-binomial coefficient $\left[\begin{array}{c}n \\ m\end{array}\right]_{q^{2}}$ also belongs to the Liskova semigroup $\mathfrak{L}^{+}$. Thus, the $q^{2}$-multinomial coefficients $\left[\begin{array}{c}N \\ m_{1}, \ldots, m_{k}\end{array}\right]_{q^{2}}$ belong to the Liskova semigroup as well.

Questions. i) Is it true that for any partition $\lambda$ the generalized $q^{2}$-binomial coefficient $\left[\begin{array}{c}n \\ \lambda\end{array}\right]_{q^{2}}$ belongs to the Liskova semigroup ?

ii) Assume that some symmetric and unimodal polynomial $P(q)$ belongs to the Liskova semigroup $\mathfrak{L}^{+}$. Is it true that the polynomial $P\left(q^{2}\right)$ also belongs to the semigroup $\mathfrak{L}^{+}$? 


\subsection{Kostka-Foulkes polynomials and transportation matrices}

Let $\lambda, \mu$ be partitions, $|\lambda|=|\mu|$, and $n, N$ be natural numbers such that $l(\lambda) \leq n, l(\mu) \leq n$, and $N \geq \lambda_{1}+\mu_{1}$. Let us define partitions $\alpha_{N}=\left(N^{n}\right)$ and

$$
\beta_{N}=\left(N-\lambda_{n}, N-\lambda_{n-1}, \ldots, N-\lambda_{1}, \mu_{1}, \mu_{2}, \ldots, \mu_{n}\right) .
$$

Theorem 7.13 Let $\lambda, \mu, n, N, \alpha_{N}$ and $\beta_{N}$ be as above. Then

i) $K_{\alpha_{N} \beta_{N}}(q) \leq K_{\alpha_{N+1} \beta_{N+1}}(q)$;

$$
\text { ii) If } N \geq|\lambda| \text {, then } K_{\alpha_{N} \beta_{N}}(q) \doteq \sum_{\eta} K_{\eta \lambda}(q) K_{\eta \mu}(q) \text {. }
$$

Identity (7.5) admits the following combinatorial interpretation. First of all, note that the value of the $\operatorname{LHS}(7.5)$ at $q=1$ is equal to the number of plane partitions $\pi$ of shape $\left(N^{n}\right)$ whose diagonal sums are fixed. More precisely, $\pi=\left(\pi_{i j}\right)_{1 \leq i<j \leq n}$ is a $n$ by $n$ matrix of non-negative integers such that

$$
\begin{aligned}
& \pi_{i j} \geq \pi_{i+1 j}, \quad 1 \leq i \leq n-1, \quad 1 \leq j \leq n ; \quad \pi_{i j} \geq \pi_{i j+1}, \\
& 1 \leq i \leq n, \quad 1 \leq j \leq n-1 ; \quad \sum_{j=1}^{k} \pi_{j, k-j+1}=\sum_{j=1}^{k} \mu_{n-k+j} ; \\
& \sum_{j=1}^{n-k+1} \pi_{n-j+1, k+j-1}=\sum_{j=k}^{n} \lambda_{j}, \quad 1 \leq k \leq n .
\end{aligned}
$$

Indeed, let $T$ be a semistandard Young tableau of shape $\left(N^{n}\right)$ and weight $\left(N-\lambda_{n}, N-\right.$ $\left.\lambda_{n-1}, \ldots, N-\lambda_{1}, \mu_{1}, \ldots, \mu_{n}\right)$. Denote by $d_{i j}, 1 \leq i \leq n, 1 \leq j \leq 2 n$, the number of cells in the $i$-th row of $T$ occupied by numbers that do not exceed $j$. Consider the diamond $\tilde{\pi}=\left(\tilde{\pi}_{i j}\right)$, where

$$
\tilde{\pi}_{i j}=N-d_{i j}, \quad 1 \leq i \leq n, \quad 1 \leq j \leq 2 n-1, \quad \text { and } \quad 0 \leq j-i \leq 2 n-1 .
$$

It is clear that $\tilde{\pi}_{i j} \geq \tilde{\pi}_{i+1, j}, \tilde{\pi}_{i j} \geq \tilde{\pi}_{i, j+1}$ and

$$
\sum_{j=1}^{k} \tilde{\pi}_{j k}=\sum_{j=1}^{k} \mu_{n-k+j}, \quad \sum_{j=k}^{n} \widetilde{\pi}_{j, n+k-1}=\sum_{j=k}^{n} \lambda_{j}, \quad 1 \leq k \leq n .
$$

If we put $\pi_{i j}=\widetilde{\pi}_{i, j-i+1}$, we will obtain the plane partition $\pi=\left(\pi_{i j}\right)_{1 \leq i, j \leq n}$ as it was defined above.

Clearly, the diamond $\tilde{\pi}$ defines two semistandard Young tableaux $P$ and $Q$ of the same shape: $P$ of weight $\overleftarrow{\lambda}=\left(\lambda_{n}, \lambda_{n-1}, \ldots, \lambda_{1}\right)$ and $Q$ of weight $\overleftarrow{\mu}=\left(\mu_{n}, \mu_{n-1}, \ldots, \mu_{1}\right)$. The statement ii) of Theorem 7.15 is equivalent to the following pure combinatorial one: the difference of charges

$$
c(T)-c(\mathbb{S}(P))-c(\mathbb{S}(Q))
$$


is a constant depending only on $n, \lambda$ and $\mu$. Here $\mathbb{S}$ denotes the Schützenberger transformation

$$
\mathbb{S}: \operatorname{STY}(\lambda, \mu) \rightarrow \operatorname{STY}(\lambda, \overleftarrow{\mu})
$$

on the set of semistandard Young tableaux of shape $\lambda$. See e.g. [45], or [13], Appendix, p.184, where the transformation $\mathbb{S}$ is called evacuation, or [30, 35].

Theorem 7.14 Let $\lambda, \mu$ be partitions, $|\lambda|=|\mu|, l(\lambda) \leq n, N \geq \lambda_{1}$. Let us define the rectangular shape partition $\alpha_{N}=\left(n^{N}\right)$, and dominant sequence of rectangular shape partitions $R_{N}=\left\{\mu,\left(1^{N-\lambda_{n}}\right), \ldots,\left(1^{N-\lambda_{1}}\right)\right\}$. Then

i) $K_{\alpha_{N} R_{N}}(q) \leq K_{\alpha_{n+1} R_{N+1}}(q)$;

$$
\text { ii) If } \quad N \geq|\lambda| \text {, then } K_{\alpha_{N} R_{N}}(q) \doteq \sum_{\eta} \bar{K}_{\eta \lambda}(q) K_{\eta^{\prime} \mu}(q)
$$

More generally, let $\beta$ be partition, $l(\beta) \leq n$, and $R, S=\left\{\left(\mu_{a}^{\eta_{a}}\right)\right\}_{a=1}^{p}$ be two dominant sequences of rectangular shape partitions such that $\sum \eta_{a}=n$, and $|S|=|R|+|\beta|$. Consider partitions $\alpha_{N}=\left(N-\beta_{n}, N-\beta_{n-1}, \ldots, N-\beta_{1}\right)$, and a dominant rearrangement $Q_{N}$ of the sequence of rectangular shape partitions $R \cup\left\{\left(N-\mu_{a}\right)^{\eta_{a}}\right\}_{a=1}^{p}$.

Theorem 7.15

i) $K_{\alpha_{N} Q_{N}}(q) \leq K_{\alpha_{N+1} Q_{N+1}}(q)$;

$$
\text { ii) If } \quad N \geq|S| \text {, then } K_{\alpha_{N} Q_{N}}(q) \doteq \sum_{\gamma} K_{\gamma \backslash \beta, R}(q) K_{\gamma, S}(q) \text {. }
$$

Similarly, let $\beta$ be partition, $l\left(\beta^{\prime}\right) \leq k$, and $R, S=\left\{\left(\mu_{a}^{\eta_{a}}\right)\right\}_{a=1}^{p}$ be two dominant sequences of rectangular shape partitions such that $\sum \mu_{a}=k$, and $|S|=|R|+|\beta|$. Consider partition $\alpha_{N}=\left(N-\beta_{k}, N-\beta_{k-1}, \ldots, N-\beta_{1}\right)^{\prime}$, and a dominant rearrangement $Q_{N}$ of the sequence of rectangular shape partitions $R \cup\left\{\left(\mu_{a}^{N-\eta_{a}}\right)\right\}_{a=1}^{p}$.

Theorem $7.16 \quad$ i) $K_{\alpha_{N} Q_{N}} \leq K_{\alpha_{N+1} Q_{N+1}}(q)$;

ii) If $\quad N \geq|S|$, then $K_{\alpha_{N} Q_{N}}(q) \doteq \sum_{\gamma} \bar{K}_{\gamma^{\prime} \backslash \beta^{\prime}, R}(q) K_{\gamma, S}(q)$.

\subsection{Gelfand-Tsetlin polytope and volume of weight subspaces}

Let $\lambda$ be a partition and $\mu$ be a composition of the same size and whose lengths do not exceed $n$. Let $G T(\lambda, \mu)$ be the convex polytope in the space $\mathbb{R}^{\frac{n(n+1)}{2}}$ of all points $\mathbf{x}=\left(x_{i j}\right)_{1 \leq i \leq j \leq n}$ satisfying the following conditions

- $x_{i n}=\lambda_{i}, 1 \leq i \leq n$;

- $x_{i, j+1} \geq x_{i j}, x_{i j} \geq x_{i+1, j+1}$, for all $1 \leq i \leq j \leq n-1$;

- $x_{11}=\mu_{1}$, and if $2 \leq j \leq n$, then $\sum_{i=1}^{j} x_{i j}-\sum_{i=1}^{j-1} x_{i j-1}=\mu_{j}$.

The polytope $G T(\lambda, \mu)$ is called Gelfand-Tsetlin polytope. The following result goes back to the original paper by Gelfand and Tsetlin (Doklady Akad. Nauk SSSR (N.S.) 71 (1950), 825-828), and was rediscovered many times. 
Proposition 7.17 Dimension $\operatorname{dim} V_{\lambda}(\mu)$ of the weight $\mu$ subspace $V_{\lambda}(\mu)$ of the irreducible highest weight $\lambda$ representation $V_{\lambda}$ of the general linear algebra $\mathfrak{g l}(n)$ is equal to the number of integer points in the Gelfand-Tsetlin polytope $G T(\lambda, \mu)$ :

$$
\operatorname{dim} V_{\lambda}(\mu)=\#\left|G T(\lambda, \mu) \cap \mathbb{Z}^{n(n+1) / 2}\right|
$$

Conjecture 7.18 (38) Let $\lambda$ and $\mu$ be partitions. All vertices of the Gelfand-Tsetlin polytope $G T(\lambda, \mu)$ have integer coordinates, i.e. $G T(\lambda, \mu)$ is a convex integral polytope.

If Conjecture 7.18 is true, then

$$
\sum_{l \geq 0} K_{l \lambda, l \mu} t^{l}=\frac{P_{\lambda \mu}(t)}{(1-t)^{d+1}}
$$

where $d=\operatorname{dim} G T(\lambda, \mu)$, and $P_{\lambda \mu}(t)$ is a polynomial with non-negative integer coefficients such that $P_{\lambda \mu}(1)$ is equal to the (normalized) volume of the Gelfand-Tsetlin polytope $G(\lambda, \mu)$.

Another consequence of Conjecture 7.18, which can be proved using the specialization $q=1$ of fermionic formula (4.2), is that the Kostka number $K_{l \lambda, l \mu}$ is a polynomial $\mathcal{E}_{\lambda \mu}(l)$ in $l$ with integer coefficients. We will call the latter by Ehrhart polynomial of the weight subspace $V_{\lambda}(\mu)$, and denote it by $\mathcal{E}_{\lambda \mu}(t)$. Hence, if $l \in \mathbb{Z}_{\geq 0}$, then

$$
\left.\mathcal{E}_{\lambda \mu}(t)\right|_{t=l}=K_{l \lambda, l \mu}
$$

It is well-known, see e.g., Exercise 6 to Section 0, that in general the Ehrhart polynomial of a convex integral polytope may have negative integer coefficients. Nevertheless, based on examples, we make a conjecture that the Ehrhart polynomial $\mathcal{E}_{\lambda \mu}(t)$ of the weight subspace $V_{\lambda}(\mu)$ has in fact non-negative integer coefficients.

Example 7.19 Let $n \geq 2$ be a positive integer. Consider partitions $\lambda=\left(n^{n}\right)$ and $\mu=$ $\left((n-1)^{n}, 1^{n}\right)$. Then the Kostka number $K_{l \lambda, l \mu}$ is equal to the number of transportation matrices of size $n$ by $n$ with row and column sums both equal to $\left(l^{n}\right)$. Thus, $\mathcal{E}_{\lambda \mu}(t)$ is the Ehrhart polynomial of the Birkhoff polytope

$$
\mathcal{B}_{n}=\left\{\left(x_{i j}\right) \in \mathbb{R}^{n^{2}} \mid \sum_{i=1}^{n} x_{i j}=1, \sum_{j=1}^{n} x_{i j}=1\right\},
$$

and $P_{\lambda \mu}(1)$ is equal to the normalized volume $\widetilde{\operatorname{vol}}\left(\mathcal{B}_{n}\right)$ of the polytope $\mathcal{B}_{n}$.

To our knowledge, the Ehrhart polynomial and volume of the Birkhoff polytope $\mathcal{B}_{n}$ are known only up to $n \leq 8$, [10]. The (normalized) leading coefficient of Ehrhart's polynomial $\mathcal{E}_{\lambda \mu}(t)$ of a weight subspace $V_{\lambda}(\mu)$ is equal to the (normalized) volume of Gelfand-Tsetlin's polytope $G(\lambda, \mu)$, and called by volume of the weight subspace $V_{\lambda}(\mu)$. It plays an important role in the theory of unitary representations of semisimple Lie groups (Harish-Chandra, Duistermaat J.J. and Heckman G.J.). 


\section{Exercises}

1. a. Let $\lambda$ be a partition, $|\lambda|=n$. Show that

$$
\sum_{l(\lambda) \leq p}\left(K_{\lambda,\left(1^{n}\right)}(q)\right)^{2}=\sum_{\nu} q^{c(\nu)} \prod_{k, j \geq 1}\left[\begin{array}{c}
P_{j}^{(k)}(\nu)+m_{j}\left(\nu^{(k)}\right) \\
m_{j}\left(\nu^{(k)}\right)
\end{array}\right]_{q},
$$

summed over all admissible configurations $\nu \in C\left(\left(n^{n}\right),\left((n-1)^{n}, 1^{n}\right)\right)$ of the square shape $\left(n^{n}\right)$ and weight $\left((n-1)^{n}, 1^{n}\right)$, such that $l\left(\nu^{(p)}\right) \leq n-p$, i.e. the number of parts of the partition $\nu^{(p)}$ does not exceed $n-p$.

For the reader's convenience, we recall the meaning of notions and symbols which were used in this Exercise (see Section 4, Definitions 4.1 and 4.2).

First of all, summation in the RHS(7.11) is taken over all sequences of partitions $\nu=$ $\left\{\nu^{(1)}, \nu^{(2)}, \ldots\right\}$ such that

- $\left|\nu^{(k)}\right|=n(n-k), 1 \leq k \leq n-1$; by definition, $\nu^{(0)}=\emptyset$;

- $P_{j}^{(k)}(\nu):=n \min (n, j+1) \delta_{k, 1}+Q_{j}\left(\nu^{(k-1)}\right)-2 Q_{j}\left(\nu^{(k)}\right)+Q_{j}\left(\nu^{(k+1)}\right) \geq 0$, for all $k, j \geq 1$.

By definition, these two conditions mean that the configuration $\nu$ is admissible of type $\left(\left(n^{2}\right) ;\left((n-1)^{n}, 1^{n}\right)\right)$, i.e. belongs to the set of admissible configurations $C\left(\left(n^{2}\right) ;\left((n-1)^{n}, 1^{n}\right)\right)$. Recall, that notation $\left((n-1)^{n}, 1^{n}\right)$ stands for the partition $(\underbrace{n-1, \ldots, n-1}_{n}, \underbrace{1, \ldots, 1}_{n})$.

- By definition, for any partition $\nu, Q_{j}(\nu)=\sum_{a} \min \left(j ; \nu_{a}\right)$ is equal to the number of boxes in the first $j$ columns of the diagram corresponding to partition $\nu$, and $m_{j}(\nu)=\nu_{j}^{\prime}-\nu_{j+1}^{\prime}$ is equal to the number of parts of partition $\nu$ of size $j$.

Secondly, the symbol $c(\nu)$ stands for the charge of configuration $\nu$, i.e.

$$
c(\nu)=\sum_{j \geq 1}\left(\begin{array}{c}
n\left(1+\delta_{j, 1}\right)-\left(\nu^{(1)}\right)_{j}^{\prime} \\
2
\end{array}\right)+\sum_{k \geq 2, j \geq 1}\left(\begin{array}{c}
\left(\nu^{(k-1)}\right)_{j}^{\prime}-\left(\nu^{(k)}\right)_{j}^{\prime} \\
2
\end{array}\right) .
$$

b. Deduce from identity (7.11) yet another combinatorial formula for Catalan numbers:

$$
\begin{aligned}
C_{n} & =\frac{(2 n) !}{n !(n+1) !}=K_{(n, n),\left(1^{n}\right)}(1) \\
& =\sum_{\beta \vdash\left(n^{2}-n\right)} \prod_{k=1}^{n-1}\left(\begin{array}{c}
(2 k+1) n-2 k-2\left(\beta_{1}+\cdots+\beta_{k}\right)+\beta_{k}-\beta_{k+1} \\
\beta_{k}-\beta_{k+1}
\end{array}\right),
\end{aligned}
$$

summed over all partitions $\beta=\left(\beta_{1} \geq \beta_{2} \geq \cdots \geq \beta_{n}\right)$ of $n^{2}-n$, such that for all $k, 1 \leq k \leq n$, the following inequalities hold

$$
\left.k(n-1) \leq \beta_{1}+\cdots+\beta_{k} \leq \frac{1}{2}\left((2 k+1) n-2 k-n \delta_{k, n}\right)\right) .
$$

The sum $\sum_{l(\lambda) \leq 2}\left(K_{\lambda,\left(1^{n}\right)}(q)\right)^{2}$ gives a new $q$-analog of the Catalan numbers which is different from those of Carlitz and Riordan [6]. 
If $p=3$, the $\operatorname{RHS}(7.11)$ with $q=1$ gives rise to a combinatorial formula for the number of vexillary permutations (see, e.g. [51], Definition (1.27)) in the symmetric group $S_{n}$.

2. (Kostant partition function)

a. Let

$$
\Sigma \subset \Phi\left(1^{n}\right)=\left\{(i, j) \in \mathbb{Z}^{2} \mid 1 \leq i<j \leq n\right\}
$$

be a subset of the set of all positive roots of type $A_{n-1}$. The $q$-analog of Kostant partition function $K_{\Sigma}(\gamma ; q)$ is defined via the decomposition

$$
\prod_{(i, j) \in \Sigma}\left(1-q x_{i} / x_{j}\right)^{-1}=\sum_{\gamma} K_{\Sigma}(\gamma ; q) x^{\gamma},
$$

summed over all sequences of integers $\gamma=\left(\gamma_{1}, \gamma_{2}, \ldots, \gamma_{n}\right) \in \mathbb{Z}^{n},|\gamma|=0$. In other words, $K_{\Sigma}(\gamma ; q)$ is the generating function for the number of ways of forming $\gamma$ as linear combinations of elements of $\Sigma$ with non-negative integer coefficients:

$$
K_{\Sigma}(\gamma ; q)=\sum_{\left\{m_{i, j}\right\}} q^{\left\|m_{i j}\right\|}
$$

where the sum is taken over all sequences of non-negative integers $\left\{m_{i j}\right\},(i, j) \in \Sigma$, such that

$$
\sum_{(i, j) \in \Sigma} m_{i j} e_{i j}=\gamma
$$

where the vector $e_{i j} \in \mathbb{Z}^{n}$ has the following components $\left(e_{i j}\right)_{k}=\delta_{i k}-\delta_{j k}, 1 \leq k \leq n$; $\|m\|=\sum_{(i, j) \in \Sigma} m_{i j}$.

i) Show that $K_{\Phi\left(1^{n}\right)}(\gamma ; 1) \neq 0$, if and only if

$$
\gamma \in Y_{n}=\left\{\begin{array}{l|l}
\left(y_{1}, \ldots, y_{n}\right) \in \mathbb{Z}^{n} & \begin{array}{l}
y_{1}+\cdots+y_{j} \geq 0, \\
y_{1}+\cdots+y_{n}=0
\end{array} \text { if } 1 \leq j \leq n,
\end{array}\right\} .
$$

ii) Show that

$$
K_{\lambda \mu \eta}(q)=\sum_{w \in S_{n}}(-1)^{l(w)} K_{\Phi(\eta)}(w(\lambda+\delta)-\mu-\delta ; q)
$$

Remark 7.20 If $\eta=\left(1^{n}\right)$, then

$$
\Phi(\eta)=\{(i, j) \mid 1 \leq i<j \leq n\}
$$

and $K_{\Phi\left(1^{n}\right)}(\gamma ; q)$ coincides with the $q$-analog of type $A$ Kostant partition function $P(\gamma ; q)$. In this case the RHS(7.14) takes the form

$$
\sum_{w \in S_{n}} \epsilon(w) P(w(\lambda+\delta)-\mu-\delta ; q)
$$

This sum is equal to the Kostka-Foulkes polynomial $K_{\lambda \mu}(q)$, see e.g. [52. Chapter III, $\S 6$, Example 4. 
iii) Let $\gamma \in Y_{n}$ and $N$ be an integer such that $N+\gamma_{n} \geq 0$. Consider partitions $\lambda_{N}=$ $N(n, n-1, \ldots, 2,1)+\gamma, \mu_{N}=N(n, n-1, \ldots, 2,1)$, and composition $\eta,|\eta|=n$. Show that

$$
K_{\Phi(\eta)}(\gamma ; q)=K_{\lambda_{N}, \mu_{N}, \eta}(q)
$$

b. Let

$$
\gamma \in Y_{n}^{+}=\left\{\gamma \in Y_{n} \mid \gamma_{i} \geq 0,1 \leq i \leq n-1\right\}, \quad n \geq 3,
$$

and $\eta=\left(1^{n-2}, 2\right)$. Show that

$$
K_{\Phi\left(1^{n}\right)}(\gamma ; 1)=\frac{1}{3}\left(\gamma_{1}+\cdots+\gamma_{n-3}+3 \gamma_{n-2}+3\right) K_{\Phi(\eta)}(\gamma ; 1) .
$$

Statement that the LHS(7.16) is divisible by the linear factor

$$
l_{n}(\gamma):=\gamma_{1}+\gamma_{2}+\cdots+\gamma_{n-3}+3 \gamma_{n-2}+3
$$

was first observed by J.R. Schmidt and A.M. Bincer, J. Math. Phys. 26 (1984), 2367-2373, see also [35]. In [35] we obtained also an explicit expression for the ratio $3 K_{\Phi\left(1^{n}\right)}(\gamma ; 1) / l_{n}(\gamma)$, from which one can see that the latter can be identified with the parabolic Kostant partition function $K_{\Phi\left(1^{n-2}, 2\right)}(\gamma ; 1)$.

c. Consider vector

$$
\gamma=\gamma_{d, n}=(d, d+1, \ldots, d+n-1,-n(2 d+n-1) / 2) \in Y_{n+1}^{+},
$$

and composition $\eta_{k}=\left(1^{k}, n-k, 1\right)$. Show that

$$
K_{\Phi\left(\eta_{k}\right)}\left(\gamma_{d, n} ; 1\right)=\prod_{p=1}^{k-1} C_{p} \prod_{1 \leq i<j \leq k} \frac{2(n-k)+i+j-1}{i+j-1} \prod_{(i, j) \in \Omega_{k, n}} \frac{2 d+i+j-1}{i+j-1}
$$

where $\Omega_{k, n}=\Phi\left(1^{n+1}\right) \backslash \Sigma_{k, n}$, and $\quad \Sigma_{k, n}=$

$$
\left\{(i, j) \mid \frac{k}{2}<i<j<\frac{2 n-k+4}{2}\right\} \backslash\left\{\left(\frac{k+1+2 a}{2}, \frac{k+3+2 a}{2}\right) \mid 0 \leq a \leq n-k\right\} .
$$

In particular,

$$
\begin{aligned}
K_{\Phi\left(1^{n+1}\right)}\left(\gamma_{d, n} ; 1\right) & =\prod_{p=1}^{n-1} C_{p} \prod_{1 \leq i<j \leq n} \frac{2 d+i+j-1}{i+j-1}, \\
K_{\Phi\left(\eta_{k}\right)}\left(\gamma_{1, n} ; 1\right) & =\prod_{p=1}^{k} C_{p} \prod_{1 \leq i<j \leq k+1} \frac{2(n-k)+i+j-1}{i+j-1}
\end{aligned}
$$

where $C_{m}:=\frac{(2 m) !}{m !(m+1) !}$ denotes the $m$-th Catalan number. 
By a theorem of Proctor [57, the product $\prod_{1 \leq i<j \leq n} \frac{2 d+i+j-1}{i+j-1}$ is equal to the number $p p^{\delta_{n}}(d)$ of (weak) plane partitions of staircase shape $\delta_{n}=(n-1, n-2, \ldots, 2,1)$ whose parts do not exceed $d$. For example, if $n=3$, there exist 5 plane partitions of shape $\delta_{3}=(2,1)$

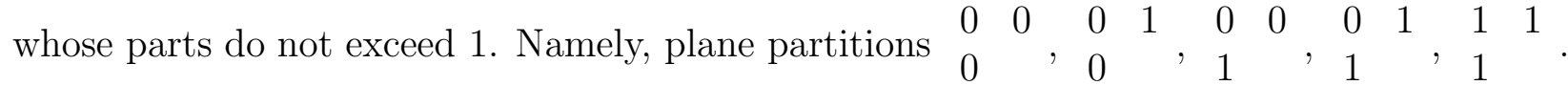
More generally, it is not difficult to see that

$$
p p^{\delta_{n}}(1)=\prod_{1 \leq i<j \leq n} \frac{i+j+1}{i+j-1}=C_{n},
$$

is equal to the $n$-th Catalan number, and

$$
\prod_{(i, j) \in \Omega_{k, n}} \frac{i+j+1}{i+j-1}=C_{k} \prod_{j=1}^{k} \frac{2 n-k+j+2}{k+j} .
$$

d. Combinatorial interpretation of identities (7.18) and (7.19).

- Let $\gamma \in Y_{n}$, and $\eta$ be a composition of $n$. Denote by $S M_{\eta}(\gamma)$ the set of $n$ by $n$ skew-symmetric integer matrices $m=\left(m_{i j}\right)$ such that

i) $m_{i j} \geq 0$, if $(i, j) \in \Phi(\eta)$;

ii) $m_{i j}=0$, if $(i, j) \in \Phi\left(1^{n}\right) \backslash \Phi(\eta)$;

iii) $\sum_{j} m_{i j}=\gamma_{i}, 1 \leq i \leq n$.

It is clear that

$$
\left|S M_{\eta}(\gamma)\right|=K_{\Phi(\eta)}(\gamma ; 1) .
$$

- Denote by $\operatorname{Cat}(n)$ the set of lattice paths from $(0,0)$ to $(n(n+1), 0)$ with steps $(1,1)$ and $(1,-1)$ never falling below the $x$-axis and passing through the points $P_{k}=(k(k+1), 0)$, $k=0,1, \ldots, n$. It is clear that the number of elements in the set $\operatorname{Cat}(n)$ is equal to the product of the first $n$ Catalan numbers $C_{1} C_{2} \cdots C_{n}$.

- Denote by $P P_{n}(d)$ the set of (weak) plane partitions of staircase shape $\delta_{n}$ whose parts do not exceed $d$.

Construct bijections

$$
\begin{aligned}
\operatorname{SM}_{\left(1^{n}\right)}\left(\gamma_{d, n-1}\right) & \simeq \operatorname{Cat}(n-2) \times P P_{n-1}(d), \\
S M_{\left(1^{k}, n-k\right)}\left(\gamma_{1, n-1}\right) & \simeq \operatorname{Cat}(k) \times \operatorname{PP}_{k+1}(n-k-1) .
\end{aligned}
$$

e. Let $n \geq 3$, consider vector

$$
\beta_{d, n}=(d, 0,1, \ldots, n-3,-d-(n-2)(n-3) / 2) .
$$

Show that

$$
K_{\Phi\left(1^{n}\right)}\left(\beta_{d, n} ; 1\right)=\prod_{j=1}^{n-3} C_{j}\left(\begin{array}{c}
d+(n-1)(n-2) / 2 \\
d
\end{array}\right) .
$$


Problem 7.21 Find a q-analog of identity (7.8), i.e. to define a statistics $L$ on the set $S M_{\left(1^{n}\right)}\left(\gamma_{d, n-1}\right)$ with the generating function

$$
\prod_{p=1}^{n-2} C(2, p \mid q) \prod_{1 \leq i<j \leq n-1} \frac{1-q^{2 d+i+j-1}}{1-q^{i+j-1}}
$$

which is agree with the decomposition (7.29).

Here

$$
C(2, m \mid q):=\frac{1-q}{1-q^{m+1}}\left[\begin{array}{c}
2 m \\
m
\end{array}\right]_{q}
$$

denotes "the most obvious" $q$-analog of the Catalan number $C_{m}$, see Section 2, Exercise 1a.

f. Show that

i) there exists a unique continuous piece wise polynomial function $\mathcal{P}_{\Phi(\eta)}\left(y_{1}, \ldots, y_{n}\right)$ on the cone $Y_{n}$ such that its restriction to the set $Y_{n} \cap \mathbb{Z}^{n}$ of integer points of the cone $Y_{n}$ coincides with the Kostant partition function $K_{\Phi(\eta)}$ :

$$
\left.\mathcal{P}_{\Phi(\eta)}(\bullet)\right|_{Y_{n} \cap \mathbb{Z}^{n}}=K_{\Phi(\eta)}(\bullet ; 1) \text {. }
$$

Polynomiality domains of the continuous piecewise polynomial function $\mathcal{P}_{\Phi(\eta)}\left(y_{1}, \ldots, y_{n}\right)$ define a subdivision of the cone $Y_{n}$ into convex integral polyhedron cones.

Question. For given $\eta$ and $n$ what is the minimal number $r_{n}(\eta)$ of polynomiality domains of the function $\mathcal{P}_{\Phi(\eta)}\left(y_{1}, \ldots, y_{n}\right)$ ? For example,

$$
r_{2}\left(\left(1^{2}\right)\right)=2, \quad r_{3}\left(\left(1^{3}\right)\right)=7, \quad r_{4}\left(\left(1^{4}\right)\right)=48 .
$$

ii) the restriction of the function $\mathcal{P}_{\Phi(\eta)}$ to the dominant chamber $Y_{n}^{+}$is a polynomial in the variables $y_{1}, \ldots, y_{n-2}$.

iii) The homogeneous degree $\left(\begin{array}{c}n-1 \\ 2\end{array}\right)$ component $\mathcal{P}_{n}^{\max }(y)$ of the polynomial $\left.\mathcal{P}_{\Phi\left(1^{n}\right)}\left(y_{1}, \ldots, y_{n}\right)\right|_{Y_{n}^{+}}$has the following form

$$
\mathcal{P}_{n}^{\max }(y)=\sum K_{\Phi\left(1^{n-2}\right)}\left(l_{1}-n+2, l_{2}-n+3, \ldots, l_{n-2}-1\right) \prod_{i=1}^{n-2} \frac{y_{i}^{l_{i}}}{l_{i} !}
$$

summed over all $(n-2)$-tuples of non-negative integers $\left(l_{1}, \ldots, l_{n-2}\right)$ such that

- $l_{1}+\cdots+l_{k} \geq k n-\frac{k(k+3)}{2}, 1 \leq k \leq n-2$,

- $l_{1}+\cdots+l_{n-2}=\frac{(n-1)(n-2)}{2}$.

Conjecture 7.22 If vector $\gamma \in \mathbb{Z}^{n}$ belongs to the cone (the so-called dominant chamber)

$$
Y_{n}^{+}=\left\{\left(y_{1}, \ldots, y_{n}\right) \in Y_{n} \mid y_{1} \geq 0, \ldots, y_{n-1} \geq 0\right\}
$$


then the normalized Kostant partition function

$$
\left(\begin{array}{c}
n-1 \\
2
\end{array}\right) ! K_{\Phi\left(1^{n}\right)}(\gamma ; 1)
$$

is an inhomogeneous polynomial of degree $\left(\begin{array}{c}n-1 \\ 2\end{array}\right)$ in the variables $\gamma_{1}, \ldots, \gamma_{n-2}$ with nonnegative integer coefficients.

Conjecture $7.23 K_{\Phi(\eta)}(\gamma ; q)$ is a unimodal polynomial in the variable $q$.

g. Show that

$$
\begin{aligned}
\operatorname{RHS}(7.18) & =\prod_{j=d}^{n+d-2} \frac{1}{2 j+1}\left(\begin{array}{c}
n+d+j \\
2 j
\end{array}\right), \\
\operatorname{RHS}(7.19) & =\prod_{j=n-k}^{n-1} \frac{1}{2 j+1}\left(\begin{array}{c}
n+1+j \\
2 j
\end{array}\right) .
\end{aligned}
$$

h. (Chan-Robbins polytope)

Let $\lambda=\left(\lambda_{1}, \ldots, \lambda_{n}\right)$ and $\mu=\left(\mu_{1}, \ldots, \mu_{n}\right)$ be compositions of the same size, without zero components, and such that

$$
\mu_{1}+\cdots+\mu_{i+1} \geq \lambda_{1}+\cdots+\lambda_{i} \text { for all } 1 \leq i \leq n-1 .
$$

Let $C R_{n}^{\lambda \mu}$ be the convex polytope in $\mathbb{R}^{n^{2}}$ of all points $\mathbf{x}=\left(x_{i j}\right)_{1 \leq i, j \leq n}$ satisfying the following conditions

- $x_{i j}=0$, if $j>i+1$;

- $\sum_{j} x_{i j}=\lambda_{i}, \sum_{i} x_{i j}=\mu_{j}$.

This is an integral polytope (i.e. all vertices of the polytope $C R_{n}^{\lambda \mu}$ have integer coordinates) of dimension $\left(\begin{array}{c}n \\ 2\end{array}\right)$. The polytope

$$
C R_{n}:=C R_{n}^{\left(1^{n}\right),\left(1^{n}\right)}
$$

was introduced and studied by Chan and Robbins [7], and Chan, Robbins and Yuen [8]. It is a face of dimension $\left(\begin{array}{l}n \\ 2\end{array}\right)$ with $2^{n-1}$ vertices of the Birkhoff polytope $\mathcal{B}_{n}$, see Example 7.19 . We will call the polytope $C R_{n}$ by Chan-Robbins polytope.

i) Show that if $k$ is a positive integer, then

$$
\begin{aligned}
i\left(C R_{n}^{\lambda \mu} ; k\right) & =\# \mid C R_{n}^{k \lambda, k \mu} \cap \mathbb{Z}^{\left(\begin{array}{c}
n \\
2
\end{array}\right)} \\
& =K_{\Phi\left(1^{n+1}\right)}\left(k \mu_{1}, k\left(\mu_{2}-\lambda_{1}\right), \ldots, k\left(\mu_{n}-\lambda_{n-1}\right),-k \lambda_{n} ; 1\right) .
\end{aligned}
$$




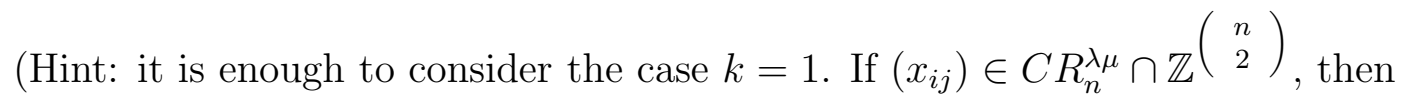

$$
\sum_{1 \leq i<j \leq n} x_{i j} e_{i, j+1}=\left(\mu_{1}, \mu_{2}-\mu_{1}, \ldots, \mu_{n}-\lambda_{n-1},-\lambda_{n}\right)
$$

and the upper triangular matrix

$$
\left(m_{i j}\right):=\left(x_{i, j-1}\right), \quad 1 \leq i<j \leq n+1
$$

defines an element of the set

$$
S M_{\Phi\left(1^{n+1}\right)}\left(\mu_{1}, \mu_{2}-\lambda_{1}, \ldots, \mu_{n}-\lambda_{n-1},-\lambda_{n}\right) .
$$

Conversely, if

$$
\left(m_{i j}\right) \in S M_{\Phi\left(1^{n+1}\right)}\left(\mu_{1}, \mu_{2}-\lambda_{1}, \ldots, \mu_{n}-\lambda_{n-1},-\lambda_{n}\right),
$$

then the point $x=\left(x_{i j}\right)_{1 \leq i, j \leq n}$, where $x_{i j}=m_{j-1, i}$ if $j \leq i+1$, and $x_{i j}=0$, if $j>i+1$, belongs to the polytope $C R_{n}^{\lambda \mu}$.)

Denote by $\mathcal{P}_{\Phi\left(1^{n+1}\right)}\left(y_{1}, \ldots y_{n+1}\right)$ a unique continuous piecewise polynomial function on the cone $Y_{n+1}$ such that

$$
\left.\mathcal{P}_{\Phi\left(1^{n+1}\right)}(\bullet)\right|_{Y_{n+1} \cap \mathbb{Z}^{n+1}}=K_{\Phi\left(1^{n+1}\right)}(\bullet ; 1),
$$

see [36], or Exercise $\mathbf{f}$ to this Section.

ii) Show that

$$
\mathcal{E}\left(C R_{n}^{\lambda \mu} ; t\right)=\mathcal{P}_{\Phi\left(1^{n+1}\right)}\left(t \mu_{1}, t\left(\mu_{2}-\lambda_{1}\right), \ldots, t\left(\mu_{n}-\lambda_{n-1}\right),-t \lambda_{n}\right),
$$

where $\mathcal{E}\left(C R_{n}^{\lambda \mu} ; t\right)$ denotes the Ehrhart polynomial of the polytope $C R_{n}^{\lambda \mu}$.

In particular, for the Chan-Robbins polytope one has

$$
\mathcal{E}\left(C R_{n} ; t\right)=\mathcal{P}_{\Phi\left(1^{n+1}\right)}(t, \underbrace{0, \ldots, 0}_{n-1},-t) .
$$

- Deduce from (7.29) and Exercise 2f, iii) to Section 7, that

$$
\widetilde{\operatorname{vol}}\left(C R_{n+1}\right)=\prod_{j=0}^{n-2} \frac{1}{j+1}\left(\begin{array}{c}
2 j \\
j
\end{array}\right)
$$

is the product of the first $n-1$ Catalan numbers. Formula (7.30) for the normalized volume of the Chan-Robbins polytope $C R_{n}$ was stated as a conjecture by Chan and Robbins [7], and has been proved by Zeilberger [72].

i. Suppose that $n \geq 3$ is an integer and that $2 \leq s \leq r \leq n-1$. Follow [8] denote by $P_{n}(r, s)$ the integral convex polytope

$$
P_{n}(r, s):=C H_{n} \cap\left\{x_{r s}=0\right\} .
$$

Denote by $p_{n}(r, s)$ the normalized volume of the polytope $P_{n}(r, s)$. 
Show that

- $p_{n}(r, 2)=K_{\Phi\left(1^{n-1}\right)}\left(\gamma_{r}^{(n-1)} ; 1\right)$, where

$$
\gamma_{r}^{(n-1)}=\left(1,2, \ldots, n-2,-\frac{(n-1)(n-2)}{2}\right)-(\underbrace{0, \ldots, 1, \ldots 0}_{n-1}), \quad 2 \leq r \leq n-1
$$

- $p_{n}(r, s)=p_{n}(r, 2)-p_{n}(s-1,2), 2 \leq s \leq r \leq n-1$;

- $p_{n}(r, 2)+p_{n}(n-r, 2)=p_{n}(n-1,2)$,

$p_{n}(r, 2)=p_{n}(n-1, n-r+1), 2 \leq r \leq n-1$,

where by definition we put $p_{n}(r, s)=0$, if $r<s$.

3. (Gelfand-Tsetlin polytope) Let $\lambda=\left(\lambda_{1} \geq \lambda_{2} \geq \cdots \geq \lambda_{r}>\lambda_{r+1}=0\right)$ and $\mu=\left(\mu_{1} \geq\right.$ $\left.\mu_{2} \geq \cdots \geq \mu_{s}>0\right)$ be partitions such that $\lambda \geq \mu$ with respect to the dominance order.

a. Show that

$$
\operatorname{dim} G(\lambda, \mu)=(r-1)(s-1)-\left(\begin{array}{l}
r \\
2
\end{array}\right)-\sum_{i=1}^{r}\left(\begin{array}{c}
\lambda_{i}^{\prime}-\lambda_{i+1}^{\prime} \\
2
\end{array}\right) .
$$

b. Denote by $a(\lambda, \mu)$ the lowest degree of $q$ which is present in $K_{\lambda \mu}(q)$, i.e.,

$$
K_{\lambda \mu}(q)=b(\lambda, \mu) q^{a(\lambda, \mu)}(1+\text { higher degree terms }) .
$$

Show that if $n \in \mathbb{Z}_{\geq 1}$, then

$$
a(n \lambda, n \mu)=n a(\lambda, \mu)
$$

Conjecture 7.24 Generating function

$$
\sum_{n \geq 0} b(n \lambda, n \mu) t^{n}
$$

is a rational function of the form $Q_{\lambda \mu}(t) /(1-t)^{r(\lambda, \mu)+1}$, where $r(\lambda, \mu) \in \mathbb{Z}_{\geq 0}$, and $Q_{\lambda \mu}(t)$ is a polynomial of degree $\leq r(\lambda, \mu)$ with non-negative integer coefficients. More generally,

$$
\sum_{k \geq 1} \sum_{n \geq 1} b((k n-1) \lambda,(k n-1) \mu) t^{k-1} z^{n-1}=Q_{\lambda \mu}(t, z) /((1-t)(1-z))^{r(\lambda, \mu)+1},
$$

where

$$
Q_{\lambda \mu}(z, t)=Q_{\lambda \mu}(t, z)
$$

is a polynomial with non-negative integer coefficients. 
Example 7.25 Take $\lambda=(7432)$ and $\mu=(4432111)$. Then $\operatorname{dim} G(\lambda, \mu)=12, a(n \lambda, n \mu)=$ $5 n$, and

$$
b(n \lambda, n \mu)=\left(\begin{array}{c}
n+3 \\
4
\end{array}\right)+\left(\begin{array}{c}
n+4 \\
4
\end{array}\right) .
$$

Hence,

$$
\begin{aligned}
& \sum_{n \geq 0} b(n \lambda, n \mu) t^{n}=\frac{1+t}{(1-t)^{5}} ; \\
& Q_{\lambda \mu}(t, z)=1+z+\left(1+15 z+6 z^{2}\right) t+\left(6 z+15 z^{2}+z^{3}\right) t^{2}+\left(z^{2}+z^{3}\right) t^{3} .
\end{aligned}
$$

c. Show that for any $n, m \in \mathbb{Z}_{\geq 0}$,

$$
K_{(n+m) \lambda,(n+m) \mu}(q) \leq K_{n \lambda, n \mu}(q) K_{m \lambda, m \mu}(q) .
$$

d. Let $\mathcal{P}$ be an integral convex polytope in $\mathbb{R}^{d}$ of dimension $d$. Show that

$$
\sum_{k_{1}, k_{2}, \ldots, k_{r} \geq 1} i\left(\mathcal{P}, k_{1} k_{2} \cdots k_{r}-1\right) \prod_{j=1}^{r} t_{j}^{k_{j}-1}=Q_{\mathcal{P}}\left(t_{1}, \ldots, t_{r}\right) / \prod_{j=1}^{r}\left(1-t_{j}\right)^{d+1},
$$

where $Q_{\mathcal{P}}\left(t_{1}, \ldots, t_{r}\right)$ is a polynomial in the variables $t_{1}, \ldots, t_{r}$ with integer coefficients. It looks an interesting combinatorial problem to describe all integral convex polytopes $\mathcal{P}$ such that the corresponding polynomial $Q_{\mathcal{P}}\left(t_{1}, t_{2}\right)$ has non-negative integer coefficients.

Question. Does there exist an integral convex polytope $\Gamma(\lambda, \mu)$ such that

$$
i(\Gamma(\lambda, \mu) ; k)=b(k \lambda, k \mu),
$$

for all integers $k \geq 1$ ? If so, is it true that in this case $\Gamma(\lambda, \mu)=\Gamma\left(\mu^{\prime}, \lambda^{\prime}\right)$ ?

e. (MacMahon polytope and Narayana numbers again) Take $\lambda=(n+k, n, n-1, \ldots, 2)$ and $\mu=\lambda^{\prime}=\left(n, n, n-1, n-2, \ldots, 2,1^{k}\right)$. Show that if $n \geq k \geq 1$, then for any positive integer $N$

- $a(N \lambda, N \mu)=(2 k-1) N$;

- $b(N \lambda, N \mu)=\operatorname{dim} V_{\left((n-k+1)^{k-1}\right)}^{\mathfrak{g} l(N+k-1)}=\prod_{i=1}^{k-1} \prod_{j=1}^{n-k+1} \frac{N+i+j-1}{i+j-1}$.

In other words, $b(N \lambda, N \mu)$ is equal to the number of (weak) plane partitions of rectangular shape $\left((n-k+1)^{k-1}\right)$ whose parts do not exceed $N$. According to Exercise 1, c, Section Q , $b(N \lambda, N \mu)$ is equal also to the number $i\left(\mathfrak{M}_{k-1, n-k+1} ; N\right)$ of rational points $\mathbf{x}$ in the MacMahon polytope $\mathfrak{M}_{k-1, n-k+1}$ such that the points $N \mathbf{x}$ have integer coordinates. Therefore, in this example one can take

$$
\Gamma(\lambda, \mu)=\mathfrak{M}_{k-1, n-k+1} .
$$

It follows from (2.23) that the generating function for numbers $b(n \lambda, n \mu)$ has the following form

$$
\sum_{n \geq 0} b(n \lambda, n \mu) t^{n}=\left(\sum_{j=0}^{(k-2)(n-k)} N(k-1, n-k+1 ; j) t^{j}\right) /(1-t)^{(k-1)(n-k+1)+1},
$$


where $N(k, n ; j), 0 \leq j \leq(k-1)(n-1)$, denote rectangular Narayana's numbers, see Section 2, Exercise 1.

- Show that if $r:=k-\left(\begin{array}{c}n+2 \\ 2\end{array}\right) \geq 0$, then $b(\lambda, \mu)=1$, and

$$
a(\lambda, \mu)=2\left(\begin{array}{c}
n+3 \\
3
\end{array}\right)+(n+1)(2 r-1)+\left(\begin{array}{l}
r \\
2
\end{array}\right)
$$

- if $1 \leq k<\left(\begin{array}{c}n+2 \\ 2\end{array}\right)$, then there exists a unique $p, 1 \leq p \leq n$, such that

$$
(p-1)(2 n-p+4) / 2<k \leq p(2 n-p+3) / 2 .
$$

In this case

$$
a(\lambda, \mu)=p(2 k-(p-1) n-p)+2\left(\begin{array}{c}
p \\
3
\end{array}\right),
$$

and one can take $\Gamma(\lambda, \mu)$ to be equal to the MacMahon polytope $\mathfrak{M}_{r(k), s(k)}$ with $r(k):=$ $k-1-(p-1)(2 n-p+4) / 2$, and $s(k):=p(2 n-p+3) / 2-k$.

This Exercise gives some flavor how intricate the piecewise linear function $a(\lambda, \mu)$ may be.

4. (Generalized saturation conjecture)

a. For any partition $\lambda$ and a dominant sequence of rectangular shape partitions $R=$ $\left(\left(\mu_{a}^{\eta_{a}}\right)\right)_{a=1}^{p}$ define the numbers $a(\lambda, R)$ and $b(\lambda, R)$ via decomposition

$$
K_{\lambda R}(q)=b(\lambda, R) q^{a(\lambda, R)}+\text { higher degree terms. }
$$

For any non-negative integer $n$ denote by $n R$ the dominant sequence of rectangular shape partitions

$$
n R:=\left(\left(n \mu_{a}\right)^{\eta_{a}}\right)
$$

Conjecture 7.26 (Generalized saturation conjecture)

$$
a(n \lambda, n R)=n a(\lambda, R) .
$$

Conjecture 7.27 i) (Generalized Fulton's conjecture) If $b(\lambda, R)=1$, then

$$
b(n \lambda, n R)=1
$$

for any non-negative integer $n$; conversely, if $b(n \lambda, n R)=1$ for some positive integer $n$, then $b(\lambda, R)=1$.

ii) More precisely, there exist non-negative integers $d$ and $a_{0}, a_{1}, \ldots, a_{d}$ (depending on $\lambda$ and $R$ ) such that $a_{0}=1$, and

$$
b(n \lambda, n R)=\sum_{k=0}^{d} a_{k}\left(\begin{array}{c}
n+k \\
d
\end{array}\right)
$$

for all $n \geq 0$. Moreover, $b(n \lambda, n R)$ is a polynomial in $n$ with non-negative rational coefficients. 
Therefore, if $b(n \lambda, n R)=1$ for some non-negative integer $n$, then $d=0$ and $a_{0}=1$, and so $b(N \lambda, N \mu)=1$ for all non-negative integers $N$.

Recall that Fulton's conjecture says that a condition $c_{\lambda \mu}^{\nu}=1$ is equivalent to $c_{N \lambda, N \mu}^{N \nu}$ being one for any $N \in \mathbb{N}$, see e.g., A. Buch (Enseign. Math. (2) 46 (2000), 43-60).

It is well-known that weight multiplicities $\operatorname{dim} V_{\lambda}(\mu)=K_{\lambda \mu}$ may be considered as a limiting case of the Littlewood-Richardson numbers, i.e. the triple tensor product multiplicities. The Fulton conjecture is a far generalization of the following (well-known) fact:

Let $\lambda$ and $\mu$ be partitions, then $K_{\lambda \mu}=1$ if and only if $K_{n \lambda, n \mu}=1$ for all positive integers $n$.

This fact is a simple corollary of the Berenstein-Zelevinsky weight-multiplicity-one criteria, see e.g., A. Berenstein and A. Zelevinsky (Functional Anal. Appl. 24 (1990), 259-269), A.N. Kirillov, Generalization of the Gale-Ryser theorem, European Journ. of Combinatorics, 21 (2000), 1047-1055, or R. Stanley [66], Exercise 7.13, p.451.

\section{Conjecture 7.28}

$$
\begin{aligned}
\text { i) } \quad a(\lambda, \mu) & =a\left(\mu^{\prime}, \lambda^{\prime}\right) ; \\
\text { ii) } \quad b(\lambda, \mu) & =b\left(\mu^{\prime}, \lambda^{\prime}\right) .
\end{aligned}
$$

Conjecture 7.28, i) was stated by I. Macdonald [52], Chapter III, §6, Example 3 on p.243. Conjecture 7.28, ii) was stated for the first time in [30], see also [35].

Let us explain briefly why Conjecture 7.26 may be considered as a generalization of saturation conjecture. First of all, let us recall a formulation of the saturation conjecture (now theorem due to A. Knutson and T. Tao, J. Amer. Math. Soc. 12 (1999), 1055-1090):

Let $\lambda, \mu, \nu$ be partitions, denote by $c_{\lambda, \mu}^{\nu}$ the corresponding Littlewood-Richardson number, i.e.

$$
c_{\lambda, \mu}^{\nu}=\operatorname{Mult}\left[V_{\nu}: V_{\lambda} \otimes V_{\mu}\right] .
$$

If for some non-negative integer $N$ one has $c_{N \lambda, N \mu}^{N \nu}=0$, then $c_{\lambda, \mu}^{\nu}=0$.

It was first shown in 1994 by A. Klyachko (Selecta Math. 4 (1998), 419-445) that the saturation conjecture implies the Horn conjecture about eigenvalues of a sum of two Hermitian matrices (A. Horn, Pacific Journ. Math. 12 (1962), 225-241). We refer the reader to survey articles by W. Fulton (Bull. Amer. Math. Soc. 37 (2000), 209-249; Séminaire Bourbaki, vol. 1997/98, Astérisque No.252 (1998), Exp. No.845, 255-269) for historical overview, description and solution of Horn's problem, and explanation of connections between Horn's and saturation conjectures.

Finally, let us show how the saturation conjecture follows from Conjecture 7.26. First of all, according to Exercise 3, Section 3, if $\lambda, \mu, \nu$ be partitions, then

$$
K_{\widetilde{\lambda}, \widetilde{R}}(q)=q^{Q_{\lambda_{1}}(\nu)-|\lambda|}\left\{c_{\lambda, \mu}^{\nu}+\text { higher degree terms }\right\} .
$$

Therefore, if $c_{N \lambda, N \mu}^{N \nu} \neq 0$ for some positive integer $N$, then

$$
a(N \widetilde{\lambda}, N \widetilde{R})=Q_{N \lambda_{1}}(N \nu)-|N \lambda|=\sum_{a} \min \left(N \lambda, N \nu_{a}\right)-N|\lambda|=N\left(Q_{\lambda_{1}}(\nu)-|\lambda|\right),
$$


and therefore,

$$
a(\widetilde{\lambda}, \widetilde{R})=Q_{\lambda_{1}}(\nu)-|\lambda| .
$$

The latter equality means that $c_{\lambda \mu}^{\nu} \neq 0$. Similarly one can show that if $c_{\lambda, \mu}^{\nu} \neq 0$ then $c_{N \lambda, N \mu}^{N \nu} \neq 0$ for any positive integer $N$.

As a byproduct, we obtain a criteria telling when the Littlewood-Richardson number $c_{\nu \mu}^{\nu} \neq 0$. Namely, let $\lambda, \mu, \nu$ be partitions such that $|\nu|=|\lambda|+|\mu|, l(\lambda) \leq p, l(\mu) \leq s$. Define partition

$$
\Lambda=\left(\lambda_{1}+\mu_{1}, \lambda_{1}+\mu_{2}, \ldots, \lambda_{1}+\mu_{s}, \lambda_{1}, \ldots, \lambda_{p}\right)
$$

and a dominant rearrangement $R$ of the sequence of rectangular shape partitions $\left\{\nu,\left(\lambda_{1}^{s}\right)\right\}$. Then $c_{\lambda \mu}^{\nu} \neq 0$ if and only if

$$
a(\Lambda, R)=Q_{\lambda_{1}}(\nu)-|\lambda|
$$

and if the latter equality holds, then

$$
c_{\lambda \mu}^{\nu}=b(\Lambda, R)
$$

In order this criteria happened to be really effective, one has to know a direct combinatorial interpretation of the numbers $a(\Lambda, R)$.

Remark 7.29 More generally, for any two partitions $\lambda$ and $\mu$ of the same size, and a composition $\eta$, such that $|\eta| \geq l(\mu)$, one can define the numbers $a(\lambda, \mu ; \eta)$ and $b(\lambda, \mu ; \eta)$ via the decomposition

$$
K_{\lambda \mu \eta}(q)=b(\lambda, \mu ; \eta) q^{a(\lambda, \mu ; \eta)}+\text { higher degree terms. }
$$

It is natural to suggest the following

Conjecture 7.30 i) $a(n \lambda, n \mu ; \eta)=n a(\lambda, \mu ; \eta)$;

ii) $b(n \lambda, n \mu ; \eta)$ is a polynomial in $n$ with non-negative rational coefficients;

iii) $b(n \lambda, n \mu ; \eta)=1$ for some positive integer $n$ if and only if $b(\lambda, \mu ; \eta)=1$;

iv) assume additionally that $|\eta| \geq \lambda_{1}$, then $a(\lambda, \mu ; \eta)=a\left(\mu^{\prime}, \lambda^{\prime} ; \eta\right)$;

$v)$ the generating function $\sum_{n \geq 0} b(n \lambda, n \mu ; \eta) t^{n}$ is a rational function in $t$ of the form

$P_{\lambda \mu \eta}(t) /(1-t)^{r(\lambda \mu \eta)+1}$, where $r(\lambda \mu \eta) \in \mathbb{Z}_{\geq 0}$ and $P_{\lambda \mu \eta}(t)$ is a polynomial of degree $\leq r(\lambda \mu \eta)$ with non-negative integer coefficients.

Let us remark that in general

$$
b(\lambda, \mu ; \eta) \neq b\left(\mu^{\prime}, \lambda^{\prime} ; \eta\right)
$$

For example, take

$$
\lambda=(7654321), \quad \mu=\left(4^{5}, 2,1^{6}\right) \text { and } \eta=(4,1,1,2,4) .
$$

Then $\mu^{\prime}=(12,6,5,5)$ and $K_{\lambda \mu \eta}(q)=q^{8}(5,17,24,17,5)$, but

$$
K_{\mu^{\prime} \lambda^{\prime} \eta}(q)=q^{8}(1,1,1) .
$$


b. Let $\lambda, \mu$ and $\nu$ be partitions. Show that

$$
a(\lambda+\nu, \mu+\nu) \leq a(\lambda, \mu),
$$

and if $l(\nu)<l(\lambda)$, then

$$
\begin{aligned}
& a(\lambda+\nu, \mu+\nu)=a(\lambda, \mu), \\
& b(\lambda+\nu, \mu+\nu) \geq b(\lambda, \mu) .
\end{aligned}
$$

See also Exercise 1 at the end of Section 8 .

Problem 7.31 Show that $a(\lambda, R)$ and $a(\lambda, \mu ; \eta)$ are (continious) piecewise linear functions of $\lambda$ and $R$, and $\lambda, \mu$ and $\eta$ correspondingly, and compute these functions explicitly.

c. Let $\lambda, \mu$ be partitions and $\nu$ be a composition such that both $\lambda+\nu$ and $\mu+\nu$ are partitions. Show that

$$
a(\lambda+\nu, \mu+\nu) \leq a(\lambda, \mu)
$$

and if $l(\nu)<l(\lambda)$, then

$$
a(\lambda+\nu, \mu+\nu)=a(\lambda, \mu) .
$$

Note that in the case under consideration the difference

$$
K_{\lambda+\nu, \mu+\nu}(q)-K_{\lambda, \mu}(q)
$$

may have negative coefficients. However, it looks plausible that all coefficients of the latter difference have the same sign.

d. (Geometrical interpretation of Conjecture 7.28) Let $\lambda$ and $\mu$ be partitions of the same size, such that $\lambda \geq \mu$, and $N$ be an integer. Follow [31, 35] consider the compact convex polytope $\Gamma^{(1)}(\lambda, \mu)$ in $\mathbb{R}^{N^{2}}$ of all points $\mathbf{x}=\left(x_{i j}\right)_{1 \leq i, j \leq N}$ satisfying the following conditions

i) $\sum_{j} x_{i j}=\lambda_{i}, \quad \sum_{i} x_{i j}=\mu_{j}^{\prime}$;

ii) $\sum_{j \leq n}\left(x_{k j}-x_{k+1, j}\right) \geq 0$ and $\sum_{i \geq k+1}\left(x_{i n}-x_{i, n+1}\right) \geq 0$ for all integers $k, n \geq 1$.

We will call this polytope by configurations polytope. One can show [35], Section 5, that the set of integer points $\Gamma_{\mathbb{Z}}^{(1)}(\lambda, \mu)$ of the configuration polytope is in one-to-one correspondence with the set $C(\lambda ; \mu)$ of admissible configurations of type $(\lambda ; \mu)$. Therefore, $\Gamma_{\mathbb{Z}}^{(1)}(\lambda, \mu) \neq 0$ if and only if $\lambda \geq \mu$.

For any $\mathbf{x} \in \mathbb{R}^{N^{2}}$ let us set

$$
c(\mathbf{x})=\sum_{i, j} x_{i j}^{2}
$$

and define

$$
\widetilde{a}(\lambda, \mu)=\min \left\{c(\mathbf{x}) \mid \mathbf{x} \in \Gamma_{\mathbb{Z}}^{(1)}(\lambda, \mu)\right\} .
$$

It easy to see that

$$
a(\lambda, \mu)=\frac{1}{2}(\widetilde{a}(\lambda, \mu)-|\lambda|) .
$$


Finally consider the convex set

$$
\Gamma_{0}^{(1)}(\lambda, \mu)=\left\{\mathbf{x} \in \Gamma^{(1)}(\lambda, \mu) \mid c(\mathbf{x})=\widetilde{a}(\lambda, \mu)\right\} .
$$

- Show that

$$
\left|\Gamma_{0}^{(1)}(\lambda, \mu) \cap \mathbb{Z}^{N^{2}}\right|=b(\lambda, \mu) .
$$

On the other hand, consider the compact convex polytope $\Gamma^{(2)}(\lambda, \mu)$ in $\mathbb{R}^{N^{2}}$ of all points $\mathbf{x}=\left(x_{i j}\right)_{1 \leq i, j \leq N}$ satisfying the following conditions

i) $\sum_{j} x_{i j}=\lambda_{i}, \quad \sum_{i} x_{i j}=\mu_{j}^{\prime}$;

ii) $\sum_{j \leq n}\left(x_{k j}-x_{k+1, j}\right) \leq \lambda_{k}-\lambda_{k+1}$ and $\sum_{i \geq k+1}\left(x_{i n}-x_{i, n+1}\right) \leq \mu_{n}^{\prime}-\mu_{n+1}^{\prime}$ for all $k, n \geq 1$.

- Show that the set $\Gamma_{\mathbb{Z}}^{(2)}(\lambda, \mu)$ of integer points of the polytope $\Gamma^{(2)}(\lambda, \mu)$ is in one-toone correspondence with the set $C\left(\mu^{\prime}, ; \lambda^{\prime}\right)$ of admissible configurations of type $\left(\mu^{\prime} ; \lambda^{\prime}\right)$, and construct such correspondence explicitly.

Finally, consider the convex set

$$
\Gamma_{0}^{(2)}(\lambda, \mu)=\left\{\mathbf{x} \in \Gamma^{(2)}(\lambda, \mu) \mid c(\mathbf{x})=\widetilde{a}(\lambda, \mu)\right\} .
$$

Conjecture 7.28 is equivalent to the following statements

- $\widetilde{a}\left(\mu^{\prime}, \lambda^{\prime}\right):=\min \left\{c(\mathbf{x}) \mid \mathbf{x} \in \Gamma_{\mathbb{Z}}^{(2)}(\lambda, \mu)\right\}=\widetilde{a}(\lambda, \mu) ;$

- $\left|\Gamma_{0}^{(2)}(\lambda, \mu) \cap \mathbb{Z}^{N^{2}}\right|=b(\lambda, \mu)$.

5. Let $\lambda$ be a partition and $R$ be a dominant sequence of rectangular shape partitions

i) Show that the generating function

$$
Q_{\lambda R}(q, t):=\sum_{n \geq 0} K_{n \lambda, n R}(q) t^{n}
$$

is a rational function in $q$ and $t$, say,

$$
Q_{\lambda R}(q, t)=\frac{N_{\lambda R}(q, t)}{D_{\lambda R}(q, t)},
$$

where the numerator $N_{\lambda R}(q, t)$ and the denominator $D_{\lambda R}(q, t)$ are suppose to be mutually prime.

ii) Let $\lambda$ and $R$ be as in i). Show that there exists the limit of cocharge parabolic Kostka polynomials

$$
\lim _{n \rightarrow \infty} \bar{K}_{n \lambda, n R}(q),
$$

where by definition

$$
\bar{K}_{\lambda R}(q)=q^{n(R)} K_{\lambda R}\left(q^{-1}\right) .
$$


Conjecture 7.32 i) The denominator of the rational function $Q_{\lambda R}(q, t)$ has a product form

$$
D_{\lambda R}(q, t)=\prod\left(1-q^{a} t\right)^{k_{a}}
$$

for some finite set of non-negative integers $\left\{k_{j_{1}}, \ldots, k_{j_{p}}\right\}$;

ii) if $b(\lambda, R)=1$, then there exists the limit of normalized parabolic Kostka polynomials

$$
\lim _{n \rightarrow \infty} q^{-n a(\lambda, R)} K_{n \lambda, n R}(q) ;
$$

iii) the generating function $\sum_{n \geq 0} K_{n \lambda, n R}(1) t^{n}$ is a rational function in $t$ of the form

$$
\frac{P_{\lambda R}(t)}{(1-t)^{d(\lambda, R)+1}}
$$

where $d(\lambda, R) \in \mathbb{Z}_{\geq 0}, P_{\lambda R}(1) \neq 0$, and $P_{\lambda R}(t)$ is a polynomial with non-negative integer coefficients.

Problem 7.33 i) Find combinatorial inerpretation(s) for the number $b(\lambda, R)$ in terms of objects similar to either the Berenstein-Zelevinsky triangles [8], or the Knutson-Tao honeycombs and hives (A. Knutson and T. Tao, [ibid]; A. Buch, Enseign. Math. (2) 46 (2000), 43-60), or the Gleiser-Postnikov web functions (O. Gleizer and A. Postnikov, Itern. Math. Res. Notes 14 (2000), 741-774), or domino tableaux (see e.g., C. Carre and B. Leclerc, Journ. Alg. Comb. 4 (1995), 201-231).

ii) Find a q-analog of numbers $b(\lambda, R)$ which generalizes the $q$-analog $c_{\lambda \mu}^{\nu}(q)$ of LittlewoodRichardson's numbers introduced by $C$. Carre and B. Leclerc [ibid].

iii) When does the number $b(\lambda, R)$ equal to 1 ?

Examples 7.34 1) Take $\lambda=(4422)$ and $R=(3,3,(2,2), 1,1)$. Then

$$
\begin{aligned}
\sum_{n \geq 0} K_{n \lambda, n R}(q) t^{n} & =\frac{\left(1+q^{4} t\right)\left(1+q^{5} t\right)}{\left(1-q^{2} t\right)\left(1-q^{3} t\right)\left(1-q^{4} t\right)^{3}\left(1-q^{6} t\right)} \\
\sum_{n \geq 0} K_{n \lambda, n R} t^{n} & =\frac{(1+t)^{2}}{(1-t)^{6}} .
\end{aligned}
$$

Note that in this case $a(\lambda, R)=2$ and $b(\lambda, R)=1$,

$$
K_{\lambda R}(q)=q^{2}(11411) \text {. }
$$

2) Take $\lambda=(52)$ and $\mu=(2221)$. Then

$$
\begin{aligned}
\sum_{n \geq 0} K_{n \lambda, n \mu}(q) t^{n} & =\frac{1+q^{5} t+q^{6} t}{\left(1-q^{4} t\right)\left(1-q^{5} t\right)\left(1-q^{7}\right)} \\
\sum_{n \geq 0} K_{n \lambda, n \mu} t^{n} & =\frac{1+2 t}{(1-t)^{3}} .
\end{aligned}
$$


Note that in this case $a(\lambda, \mu)=4$ and $b(\lambda, \mu)=1$.

3) Take $\lambda=(422)$ and $\mu=(22211)$. Then

$$
\begin{aligned}
\sum_{n \geq 0} K_{n \lambda, n \mu}(q) t^{n} & =\frac{1+\left(q^{4}+q^{5}+q^{6}\right) t-\left(q^{8}+q^{9}+q^{10}\right) t^{2}-q^{14} t^{3}}{\left(1-q^{3} t\right)^{2}\left(1-q^{4} t\right)\left(1-q^{5} t\right)^{2}\left(1-q^{7} t\right)} \\
\sum_{n \geq 0} K_{n \lambda, n \mu} t^{n} & =\frac{1+4 t+t^{2}}{(1-t)^{5}}
\end{aligned}
$$

Note that in this case $a(\lambda, \mu)=3$ and $b(\lambda, \mu)=2$.

4) Take $\lambda=(732)$ and $\mu=(432111)$. Then

$$
\sum_{n \geq 0} K_{n \lambda, n \mu} t^{n}=\frac{1+34 t+189 t^{2}+228 t^{3}+57 t^{4}+2 t^{5}}{(1-t)^{8}} .
$$

Here $a(\lambda, \mu)=5$ and $b(\lambda, \mu)=3$, since $K_{\lambda \mu}(q)=q^{5}(3,7,9,9,7,4,2,1)$.

It is not difficult to check that the Gelfand-Tsetlin polytope $G(\lambda, \mu)$ is an integral one, and its normalized volume is equal to 511.

5) Take $\lambda=(44), \mu=\left(1^{8}\right)$. Then

$$
\sum_{n \geq 0} K_{n \lambda, n \mu}(q)=\frac{N_{\lambda \mu}(q, t)}{D_{\lambda \mu}(q, t)}
$$

where

$$
\begin{aligned}
N_{\lambda \mu}(q, t) & =1+q^{16}\left(1+q+q^{2}+q^{3}+q^{4}+q^{5}+q^{6}\right)\left(t-q^{53} t^{4}\right) \\
& +q^{33}\left(1+q+q^{2}+q^{3}+2 q^{4}+2 q^{5}+2 q^{6}+q^{7}+q^{8}\right. \\
& \left.+q^{9}+q^{10}\right)\left(t^{2}-q^{15} t^{3}\right)-q^{91} t^{5} ; \\
D_{\lambda \mu}(q, t) & =\left(1-q^{12} t\right)\left(1-q^{14} t\right)\left(1-q^{15} t\right)\left(1-q^{16} t\right)\left(1-q^{18} t\right) \\
& \cdot\left(1-q^{20} t\right)\left(1-q^{24} t\right) ; \\
\sum_{n \geq 0} K_{n \lambda, n \mu} t^{n} & =\frac{1+8 t+22 t^{2}+8 t^{3}+t^{4}}{(1-t)^{6}} .
\end{aligned}
$$

In this example

$$
K_{\lambda \mu}(q)=q^{12}(1011212121101),
$$

$\operatorname{dim} G(\lambda, \mu)=5$, and the normalized volume $\widetilde{\operatorname{vol}} G(\lambda, \mu)=40$.

6) Take $\lambda=(333), \mu=\left(1^{9}\right)$. Then

$$
\sum_{n \geq 0} K_{n \lambda, n \mu} t^{n}=\frac{1+31 t+469 t^{2}+2113 t^{3}+3466 t^{4}+2113 t^{5}+469 t^{6}+31 t^{7}+t^{8}}{(1-t)^{11}} .
$$

In this example, $K_{\lambda \mu}=42, a(\lambda, \mu)=9, b(\lambda, \mu)=1, \operatorname{dim} G(\lambda, \mu)=10$. It is not difficult to check that the Gelfand-Tsetlin polytope $G(\lambda, \mu)$ is an integral one, and its normalized volume is equal to 8694 . 
7) Take $\lambda=\left(52^{3} 1\right), \mu=\left(2^{6}\right)$. One can check that there exists only one admissible configuration of type $(\lambda ; \mu)$, i.e. $|C(\lambda ; \mu)|=1$. On the other hand $|C(2 \lambda ; 2 \mu)|=2$. This observation shows that in the case under consideration the configurations polytope $\Gamma^{(1)}(\lambda, \mu)$ cannot be an integral polytope, but only a rational one.

It is not difficult to check that

$$
\begin{aligned}
K_{n \lambda, n \mu}(q) & =q^{4 n}\left[\begin{array}{c}
2 n+4 \\
3
\end{array}\right]_{q}\left[\begin{array}{c}
n+3 \\
3
\end{array}\right]_{q} \frac{1-q^{n+1}}{1-q^{4}} \\
\sum_{n \geq 0} K_{n \lambda, n \mu} t^{n} & =\frac{1+32 t+128 t^{2}+104 t^{3}+15 t^{4}}{(1-t)^{8}}
\end{aligned}
$$

Therefore, $\operatorname{dim} G(\lambda, \mu)=7, \widetilde{\operatorname{vol}} G(\lambda, \mu)=280$, and

$$
\lim _{n \rightarrow \infty} q^{-n a(\lambda, \mu)} K_{n \lambda, n \mu}(q)=\frac{1}{(q ; q)_{3}(q ; q)_{4}} .
$$

6. Example of a convex integral polytope such that its Ehrhart's polynomial has negative integer coefficient.

a. Let $\tau_{r} \subset \mathbb{R}^{3}$ be the tetrahedron whose vertices are the points $(0,0,0),(1,0,0),(0,1,0)$ and $(1,1, r)$, where $r$ is a positive integer.

Show that

- $\mathcal{E}\left(\tau_{r} ; t\right)=\left(r t^{3}+6 t^{2}+(12-r) t+6\right) / 6$;

- $\sum_{n \geq 0} i\left(\tau_{r} ; n\right) t^{n}=\frac{1+(r-1) t^{2}}{(1-t)^{4}}$.

Hence, the tetrahedron $\tau_{r}$ contains no lattice points other than its vertices, has volume $r / 6$, and if $r>12$, then the Ehrhart polynomial $\mathcal{E}\left(\tau_{r} ; t\right)$ has negative coefficient. Note that the $\delta$-vector of the tetrahedron $\tau_{r}$ is equal to $(1,0, r-1)$, which is not unimodal.

This example is due to J. Reeve (Proc. London Math. Soc. 7 (1957), 378-395). Note also, see [25], p. 111, that there exists an integral simplex $\Delta \subset \mathbb{R}^{8}$ of dimension 8 , which satisfies the condition $(\Delta-\partial \Delta) \cap \mathbb{Z}^{8} \neq \emptyset$, and such that its $\delta$-vector is equal to $\delta(\Delta)=$ $(1,8,15,13,15,14,14,14,5)$, which is not unimodal.

Question. Is it true or not that if the $\delta$-vector of a convex integral polytope $\mathcal{P}$ is unimodal, then the Ehrhart polynomial $\mathcal{E}(\mathcal{P} ; t)$ of the polytope $\mathcal{P}$ has non-negative integer coefficients?

b. Compute the Ehrhart polynomial and $\delta$-vector of the simplex in the space $\mathbb{R}^{d}$ with the following vertices

$$
(\underbrace{0, \ldots, 0)}_{d}, e_{1}, \ldots, e_{d-1},(\underbrace{1, \ldots, 1}_{d-1}, r)
$$

where $e_{i}=\left(e_{i k}\right)_{k=1}^{d}$, and $e_{i k}=\delta_{i, k}$.

7. Let $\lambda$ be a partition, $\lambda=\left(p_{1}^{m_{1}}, \ldots, p_{k}^{m_{k}}\right)$, where $p_{1}>p_{2}>\cdots>p_{k}>0$ and each $m_{i} \geq 1$. Denote by $Y_{k}(\lambda)$ the convex (non-compact) polyhedron in $\mathbb{R}^{k+1}$ of all points $\mathbf{y}=\left(y_{1}, \ldots, y_{k+1}\right)$ satisfying the following conditions

- $y_{1}+\cdots+y_{i} \geq 0$ for all integers $i, 1 \leq i \leq k$;

- $-m_{i} \leq y_{i} \leq p_{i-1}-p_{i}$ for all integers $i, 2 \leq i \leq k$; 
- $y_{1}+\cdots+y_{k+1}=0$.

i) Show that the set of integer points of the polyhedron $Y_{k}(\lambda)$ is in one-to-one correspondence with the set of vexillary permutations $w \in S_{\infty}$ of shape $\lambda(w)=\lambda$.

ii) Denote by $Y_{k}(\lambda ; n)$ the convex polytope in $\mathbb{R}^{k+1}$ of all points $\mathbf{y} \in Y_{k}(\lambda)$ that additionally satisfy the following condition

$$
p_{i}+y_{1}+\cdots+y_{i}+m_{1}+\cdots+m_{i} \leq n
$$

for all integers $i, 1 \leq i \leq k$.

Show that the number of integer points of the (rational) polytope $Y_{k}(\lambda ; n)$ is equal to the number of vexillary permutation $w \in S_{n}$ of shape $\lambda(w)=\lambda$. We refer the reader to [51], Chapter I, for definition and basic properties of vexillary permutations. Note that formula (7.11) with $p=3$ gives an explicit combinatorial expression for the number of vexillary permutations in the symmetric group $S_{n}$.

8. Let $P(q)$ be a polynomial with non-negative integer coefficients.

i) Show that there exists a non-negative integer $N$, depending on $P(q)$, such that the product $(1+q)^{N} P(q)$ is a unimodal polynomial.

We denote by $u d(P)$ the least non-negative integer $N$ with this property.

ii) Show that if $n \geq 2$, then

$$
u d\left(1+q^{n}\right)=n^{2}-3
$$

\section{Stable behavior of Kostka-Foulkes polynomials}

Given partitions $\alpha$ and $\beta$ with $l(\alpha)+l(\beta) \leq n$, define

$$
V_{\alpha, \beta}:=V_{\alpha, \beta}^{[n]}
$$

as the Cartan piece in $V_{\alpha} \otimes V_{\beta}^{*}$, i.e. the irreducible $\mathfrak{g} l(n)$-submodule generated by the tensor product of the highest weight vectors in each factor. It is well-known that $V_{\alpha, \beta}^{[n]}=V_{\gamma}^{[n]}$ for

$$
\gamma:=[\alpha, \beta]_{n}=(\alpha_{1}+\beta_{1}, \ldots, \alpha_{r}+\beta_{1}, \underbrace{\beta_{1}, \ldots, \beta_{1}}_{n-r-s}, \beta_{1}-\beta_{s}, \ldots, \beta_{1}-\beta_{2}),
$$

where $r=l(\alpha)$ and $s=l(\beta)$. For example,

$$
V_{(0),(0)}^{[n]}=\mathbb{C}, \quad V_{(1),(1)}^{[n]}=\mathfrak{g} \quad(\text { the adjoint representation }) .
$$

In Sections 5 and 6 we had investigated the properties of the mixed tensor representations $V_{[\alpha, \beta]_{n}}$ and their zero-weight subspaces. In this Section we are going to study the stable behaviour of more general families of weight subspaces in $V_{[\alpha, \beta]_{n}}$.

Let $\lambda, \mu, \nu$ be partitions, $|\lambda|=|\mu|$, consider a family of partitions $\lambda_{n}:=\lambda \oplus \nu^{n}$ and $\mu_{n}:=\mu \oplus \nu^{n}$. Recall that if $\lambda$ and $\mu$ be partitions, then $\lambda \oplus \mu$ denotes the partition whose parts are those of $\lambda$ and $\mu$, arranged in descending order, and $\mu \oplus \nu^{n}=\mu \oplus \underbrace{\nu \cdots \oplus \nu}_{n}$. 
Proposition 8.1 ([30, 35]) i) The number $\#\left|C\left(\lambda_{n}, \mu_{n}\right)\right|$ of admissible configurations of type $\left(\lambda_{n} ; \mu_{n}\right)$ is finite and independent on $n$ if $n$ is big enough.

ii) ("Gupta conjecture") If $n \geq 0$, then

$$
K_{\lambda_{n+1}, \mu_{n+1}}(q) \geq K_{\lambda_{n}, \mu_{n}}(q)
$$

i.e. the difference $K_{\lambda_{n+1}, \mu_{n+1}}(q)-K_{\lambda_{n}, \mu_{n}}(q)$ is a polynomial with non-negative integer coefficients.

iii) There exist the limits

$$
\begin{aligned}
\lim _{n \rightarrow \infty} K_{\lambda_{n}, \mu_{n}}(q) & =Z_{\lambda \mu}^{\nu}(q), \\
\lim _{n \rightarrow \infty} \bar{K}_{\lambda_{n}, \mu_{n}}(q) & =Y_{\lambda \mu}^{\nu}(q),
\end{aligned}
$$

which are rational functions of $q$.

Remark 8.2 The statement ii) of Proposition 8.1 was stated as a conjecture by Gupta 21], and has been proved by G.-N. Han [22], and A.N. Kirillov 30]. The proof given in 22, was based on the explicit combinatorial construction of an embedding

$$
\operatorname{STY}(\lambda, \mu) \hookrightarrow S T Y(\lambda \oplus(a), \mu \oplus(a))
$$

of the set $\operatorname{STY}(\lambda, \mu)$ of semistandard Young tableaux of shape $\lambda$ and weight $\mu$ to that $\operatorname{STY}(\lambda \oplus(a), \mu \oplus(a))$ of semistandard tableaux of shape $\lambda \oplus(a)$ and weight $\mu \oplus(a)$. The proof given in [30] was based on the theory of rigged configurations. It is still an open question whether or not a bijection constructed by G.-N. Han [22] is compatible with the rigged configurations bijection.

We are going to describe the rational functions $Z_{\lambda \mu}^{\nu}(q)$ and $Y_{\lambda \mu}^{\nu}(q)$ in a particular case when the partition $\nu$ consists of one part, namely, $\nu=(a)$.

Without lost of generality, one can assume that partitions $\lambda$ and $\mu$ have the following forms

$$
\begin{aligned}
& \lambda=(\alpha_{1}+a, \ldots, \alpha_{r}+a, \underbrace{a, \ldots, a}_{k-r-s}, a-\beta_{s}, a-\beta_{s-1}, \ldots, a-\beta_{1}), \\
& \mu=(\gamma_{1}+a, \ldots, \gamma_{l}+a, \underbrace{a, \ldots, a}_{k-l-p}, a-\delta_{p}, a-\delta_{p-1}, \ldots, a-\delta_{1}),
\end{aligned}
$$

where $\alpha=\left(\alpha_{1}, \ldots, \alpha_{r}\right), \beta=\left(\beta_{1}, \ldots, \beta_{s}\right), \gamma=\left(\gamma_{1}, \ldots, \gamma_{l}\right)$, and $\delta=\left(\delta_{1}, \ldots, \delta_{p}\right)$ are partitions such that $|\alpha|+|\delta|=|\beta|+|\gamma|, l(\alpha)+l(\beta)=l(\gamma)+l(\delta) \geq k$.

We start with a study of the simplest case when $\gamma=\delta=\emptyset$, i.e. $\mu=\left(a^{k}\right)$. In this case one can use Corollaries 4.4 and 6.7 to conclude that

$$
\lim _{n \rightarrow \infty} K_{\lambda \oplus\left(a^{n}\right),\left(a^{n+k}\right)}(q)=\lim _{n \rightarrow \infty} s_{\alpha} * s_{\beta}\left(q, \ldots, q^{n+k-1}\right)=q^{|\alpha|} s_{\alpha} * s_{\beta}\left(1, q, q^{2}, \ldots\right),
$$


where $\alpha=\left(\left(\lambda_{1}-a\right)_{+},\left(\lambda_{2}-a\right)_{+}, \ldots\right), \beta=\left(\left(a-\lambda_{k}\right)_{+},\left(a-\lambda_{k-1}\right)_{+}, \ldots\right)$, and the symbol $(a)_{+}$ stands for $\max (a, 0)$.

The principal specialization $s_{\alpha} * s_{\beta}\left(1, q, q^{2}, \ldots\right)$ has been computed by Stanley [64]. Combining with [52], Chapter VI, $\S 8$, Exercise 3, the Stanley result may be stated as follows

$$
s_{\alpha} * s_{\beta}\left(1, q, q^{2}, \ldots\right)=\frac{K_{\beta \alpha}(q, q)}{H_{\alpha}(q)} .
$$

Summarizing, we obtain the following result

$$
\lim _{n \rightarrow \infty} K_{\lambda \oplus\left(a^{n}\right),\left(a^{n+k}\right)}(q)=q^{|\alpha|} \frac{K_{\beta \alpha}(q, q)}{H_{\alpha}(q)}
$$

where $H_{\alpha}(q)$ denotes the hook polynomial, see e.g. [52], Chapter I, §3, Example 3, and $K_{\beta \alpha}(q, q)$ denotes the specialization $q=t$ of the double Kostka-Macdonald polynomial $K_{\beta \alpha}(q, t)$, 52, Chapter VI, $\S 8$.

As for the limit $n \rightarrow \infty$ of the cocharge Kostka-Foulkes polynomials $\bar{K}_{\lambda \oplus\left(a^{n}\right),\left(a^{n+k}\right)}(q)$, it follows from Corollary 4.4 that

$$
\lim _{n \rightarrow \infty} \bar{K}_{\lambda \oplus\left(a^{n}\right),\left(a^{n+k}\right)}(q)=\frac{\bar{K}_{\beta,(1|\beta|)}(q)}{H_{\alpha}(q)} .
$$

The next case we are going to consider is the case when $\gamma=\emptyset$, i.e. $\mu_{1} \leq a$.

Theorem 8.3 Let $\lambda$ and $\mu$ be partitions of the forms (8.2) and (8.3) correspondingly, and assume additionally that $\mu_{1} \leq a$. Then

$$
\begin{aligned}
\lim _{n \rightarrow \infty} K_{\lambda \oplus\left(a^{n}\right), \mu \oplus\left(a^{n}\right)}(q) & =\frac{q^{|\alpha|}}{H_{\alpha}(q)} \sum_{\eta} K_{\eta \alpha}(q, q) K_{\beta \backslash \eta, \delta}(q) ; \\
\lim _{n \rightarrow \infty} \widetilde{K}_{\lambda \oplus\left(a^{n}\right), \mu \oplus\left(a^{n}\right)}(q) & =\frac{\widetilde{K}_{\beta,\left(\delta, 1^{|\alpha|}\right)}(q)}{H_{\alpha}(q)} .
\end{aligned}
$$

Example 8.4 Take $\lambda=(7,3,2), \mu=(4,3,2,1,1,1)$ and $a=(4)$. Then we have $\alpha=(3)$, $\beta=(4,4,4,2,1)$ and $\delta=(3,3,3,2,1)$. The Kostka-Foulkes polynomials $K_{\lambda \oplus\left(a^{n}\right), \mu \oplus\left(a^{n}\right)}(q)$ have been computed in [35], $\S 7$, Example 6, as well as the limits

$$
\begin{aligned}
& \lim _{n \rightarrow \infty} K_{\lambda \oplus\left(a^{n}\right), \mu \oplus\left(a^{n}\right)}(q)=q^{5} \frac{6+11 q+14 q^{2}+13 q^{3}+9 q^{4}+3 q^{5}+2 q^{6}}{(1-q)^{2}\left(1-q^{3}\right)}, \\
& \lim _{n \rightarrow \infty} \widetilde{K}_{\lambda \oplus\left(a^{n}\right), \mu \oplus\left(a^{n}\right)}(q)= \\
& \frac{1+q+3 q^{2}+5 q^{3}+7 q^{4}+9 q^{5}+11 q^{6}+8 q^{7}+7 q^{8}+4 q^{9}+q^{10}+q^{11}}{(1-q)^{2}\left(1-q^{3}\right)} .
\end{aligned}
$$

First of all, the hook polynomial

$$
H_{\alpha}(q)=(1-q)\left(1-q^{2}\right)\left(1-q^{3}\right)
$$


and using the fermionic formula (4.2) for Kostka-Foulkes polynomials, it is not difficult to check that

$$
\begin{aligned}
K_{\beta,(\delta, 1,1,1)}(q) & =q^{3}(1,2,5,11,15,19,20,16,12,8,4,2,1) \\
& =q^{3}(1+q)(1,1,4,7,8,11,9,7,5,3,1,1)
\end{aligned}
$$

Hence,

$$
\lim _{n \rightarrow \infty} \widetilde{K}_{\lambda \oplus\left(a^{n}\right), \mu \oplus\left(a^{n}\right)}(q)=\frac{\widetilde{K}_{\beta,(\delta, 1,1,1)}(q)}{H_{\alpha}(q)},
$$

as it has to be according to Theorem 8.3, (8.7).

On the other hand,

$$
\begin{aligned}
& \sum_{\eta} K_{\beta \backslash \eta, \delta}(q) K_{\eta \alpha}(q, q)=K_{(44421) \backslash(21), \beta}(q) K_{(21), \alpha}(q, q) \\
+\quad & K_{(44421) \backslash(3), \beta}(q) K_{(3), \alpha}(q, q)+K_{(44421) \backslash\left(1^{3}\right), \beta}(q) K_{\left(1^{3}\right), \alpha}(q, q) .
\end{aligned}
$$

Now one can check that

$$
\begin{aligned}
K_{(44421) \backslash(21), \beta}(q) & =q(3,8,10,10,6,2,1), \quad K_{(21), \alpha}(q, q)=q+q^{2} ; \\
K_{(44421) \backslash(3), \beta}(q) & =q^{2}(3,5,5,3,1), \quad K_{(3), \alpha}(q, q)=1 ; \\
K_{(44421) \backslash\left(1^{3}\right), \beta}(q) & =(1,2,4,5,4,2,1), \quad K_{\left(1^{3}\right), \alpha}(q, q)=q^{3} .
\end{aligned}
$$

Therefore,

$$
\begin{aligned}
\sum_{\eta} K_{\beta \backslash \eta, \delta}(q) K_{\eta \alpha}(q, q) & =q^{2}(6,17,25,27,22,12,5,2) \\
& =q^{2}(1+q)(6,11,14,13,9,3,2)
\end{aligned}
$$

as it should be according to Theorem 8.3, 8.6).

Finally, we consider the general case:

Theorem 8.5 Let $\lambda$ and $\mu$ be partitions of the forms (8.9) and (8.9) respectively, then

$$
\begin{aligned}
& \lim _{n \rightarrow \infty} K_{\lambda \oplus\left(a^{n}\right), \mu \oplus\left(a^{n}\right)}(q) \doteq \\
& \frac{1}{(q ; q)_{|\alpha|-|\gamma|}} \sum_{\eta_{1}, \eta_{2}} K_{\beta \backslash \eta_{1}, \delta}(q) \bar{K}_{\alpha \backslash \eta_{2}, \gamma}(q) K_{\eta_{1}, \eta_{2}}(q, q) K_{\eta_{2},(1|\alpha|+|\gamma|)}(q) ; \\
& \lim _{n \rightarrow \infty} \bar{K}_{\lambda \oplus\left(a^{n}\right), \mu \oplus\left(a^{n}\right)}(q) \doteq \frac{1}{(q ; q)_{|\alpha|-|\gamma|}} \bar{K}_{\alpha,(\gamma \oplus(1|\alpha|-|\gamma|))}(q) \bar{K}_{\beta,(\delta \oplus(1|\beta|-|\delta|))}(q) .
\end{aligned}
$$




\section{Exercises}

1. Let $\lambda$ and $\mu$ be partitions, define $\lambda+\mu$ to be the sum of the sequences $\lambda$ and $\mu$ :

$$
(\lambda+\mu)_{i}=\lambda_{i}+\mu_{i}
$$

The operations + and $\oplus$ are dual to each other, i.e. $(\lambda \oplus \mu)^{\prime}=\lambda^{\prime}+\mu^{\prime}$.

Show that if $\nu$ is a partition, then

$$
K_{\lambda+\nu, \mu+\nu}(q) \geq K_{\lambda, \mu}(q)
$$

Conjecture 8.6 Let $\lambda, \mu$ and $\nu$ be partitions, then

$$
K_{\lambda \oplus \nu, \mu \oplus \nu}(q, t) \geq K_{\lambda, \mu}(q, t),
$$

i.e. the difference $K_{\lambda \oplus \nu, \mu \oplus \nu}(q, t)-K_{\lambda, \mu}(q, t)$ is a polynomial in $q$ and $t$ with non-negative (integer) coefficients.

Here $K_{\lambda, \mu}(q, t)$ stands for the double Kostka polynomial introduced by I. Macdonald, 52, Chapter VI, (8.11). Note that Conjecture 8.6 gives a common (conjectural) generalization of inequalities (8.1) and (8.8).

Question. Does there exist the limit $\lim _{n \rightarrow \infty} K_{\lambda \oplus \nu^{n}, \mu \oplus \nu^{n}}(q, t)$ ?

2. For given integers $n \geq 1$ and $l \geq 1$, let $\lambda$ and $\mu$ be partitions such that $|\lambda| \equiv|\mu|(\bmod n)$ and $\mu_{1} \leq l$. Consider two sequences of partitions

$$
\lambda_{L}:=\lambda+\left(\left(L l+\frac{|\mu|-|\lambda|}{n}\right)^{n}\right) \text { and } \mu_{L}:=\left((l)^{n L}, \mu\right) .
$$

Show that there exist the limits $\lim _{L \rightarrow \infty} K_{\lambda_{L}, \mu_{L}}(q)$ and $\lim _{L \rightarrow \infty} \bar{K}_{\lambda_{L}, \mu_{L}}(q)$, which are formal power series in $q$, but both do not equal to any rational function.

Problem 8.7 Find combinatorial and representation theoretical interpretations of the latter power series.

For partial answer on this problem see [26, 33, 55].

\section{Acknowledgments}

This notes grew out of the series of lectures given at the Hokkaido, Kyushu, and Nagoya Universities, and at the RIMS and IIAS (Kyoto). I would like to thank those who attended for helpful comments, suggestions and support. In particular M. Kashiwara, T. Miwa, H. Umemura, H.-F. Yamada and M. Yoshida. My special thanks go to Alexander Postnikov for very helpful discussions. I wish thank with much gratitude the colleagues at the Graduate School of Mathematics, Nagoya University, for their hospitality which made it possible to finish this work.

I would like to acknowledge my special indebtedness to Dr. N.A. Liskova for the inestimable help and support on all stages of the paper writing. 


\section{References}

[1] Andrews G., The Theory of Partitions, Addison-Wesley Publ. Company, 1976.

[2] Berenstein A.D. and Zelevinsky A.V., Triple multiplicities for sl $(r+1)$ and the spectrum of the exterior algebra of the adjoint representation, Journ. Algebraic Comb. 1 (1992), $7-22$.

[3] Brylinski R.K., Stable calculus of the mixed tensor character I, Seminaire d'Algebre Paul Dubreil et Marie-Paul Malliavin, 39eme Annee (Paris, 1987/1988), 35-94, Lecture Notes in Math. 1404.

[4] Brylinski R.K., Matrix concomitants with mixed tensor model, Adv. in Math. 100 (1993), $28-52$.

[5] Butler L., Subgroup Lattices and Symmetric Functions, Memoirs of AMS 539 (1994).

[6] Carlitz L. and Riordan J., Two element lattice permutation numbers and their qgeneralization, Duke Math. J. 31 (1964), 371-388.

[7] Chan C.S. and Robbins D.P., On the volume of the polytope of doubly stochastic matrices, Experiment. Math. 8 (1999), no.3, 291-300.

[8] Chan C.S., Robbins D.P. and Yuen D.S., On the volume of a certain polytope, Experiment. Math. 9 (2000), no.1, 91-99.

[9] Donin I.F., Decompositions of tensor products of representations of a symmetric group and of symmetric and exterior powers of the adjoint representation of $\operatorname{sl}(N)$, Soviet Math. Dokl. 38 (1989), 654-658.

[10] Désarménien J., Leclerc B. and Thibon J.-Y., Hall-Littlewood functions and KostkaFoulkes polynomials in representation theory, Seminaire Lotharingien de Combinatoire, 32 (1994)(electronic).

[11] Egecioglu O. and Remmel J.B., Combinatorial interpretation of the inverse Kostka matrix, Linear and Multilinear Alg. 26 (1990), 59-84.

[12] Foulkes H.O., A survey of some combinatorial aspects of symmetric functions, in Permutations, Cauthier-Villas, Paris, 1974, 79-92.

[13] Fulton W., Young Tableaux, Cambridge Univ. Press, Cambridge, 1997.

[14] Fürlinger J. and Hofbauer J., q-Catalan numbers, Journ. of Comb. Theory A 40 (1985), 248-264.

[15] Garsia A.M. and Procesi C., On certain graged $S_{n}$-modules and the $q-K o s t k a$ polynomials, Adv. Math. 94 (1992), 82-138. 
[16] Gessel I. and Viennot G., Determinants, paths, and plaine partitions, Manuscript dated by $7 / 28 / 1989$.

[17] Goodman F., O'Hara K. and Stanton D., A unimodality for a Schur functions, Journ. Comb. Theory, Ser. A 80 (1992), 143-146.

[18] Green J.A., The characters of the finite general group, Trans. Amer. Math. Soc. 80 (1955), 402-447.

[19] Green J.A., Les polynômes de Hall et les charactéres des groupes $G L(n, q)$, Colloque d'algébra supérieure, Brussels, 1956, 207-215; Labraire Gouthier-Villars, Paris, 1957.

[20] Gupta R.K., Generalized exponents via Hall-Littlewood symmetric functions, Bull. Amer. Math. Soc. 16 (1987), 287-291.

[21] Gupta R.K., Problem 9 of Problem session, Contemporary Math. 34, 309; Amer. Math. Soc., Providence, R.I., 1984.

[22] Gupta R.K. and Hanlon P., Problem 5 of Problem session, Contemporary Math. 34, 305-307; Amer. Math.Soc., Providence, R.I., 1984.

[23] Han G,-N., Croissance des polynômes de Kostka, C.R. Acad. Sci. Paris 311 (1990), 269-272.

[24] Hanlon P., On the decomposition of the tensor algebra of the classical Lie algebras, Adv. in Math. 56 (1985), 238-282.

[25] Hibi T., Algebraic combinatorics on convex polytope, Carslaw Publications, 1992.

[26] Hatayama G., Kirillov A.N., Kuniba A., Okado M., Takagi T. and Yamada Y., Character formulae of $\widehat{s} l(n)$-modules and inhomogeneous paths, Nucl. Phys. B536 (1999), 575-616.

[27] Kac V., Infinite dimensional Lie algebras, 3rd ed., Cambridge Univ. Press, 1994.

[28] Kato S., Spherical functions and a q-analogue of Kostant's weight multiplicity formula, Invent. Math. 66 (1982), 461-468.

[29] Kirillov A.N., Completeness of the states for the generalized Heisenberg model (Russian), Zap. Nauch. Semin. LOMI 134 (1985), p.169-189, translation in Journal of Soviet Math., 36 (1987), p.115-128.

[30] Kirillov A.N., On the Kostka-Green-Foulkes polynomials and Clebsch-Gordon numbers, Journ. Geom. and Phys. 5 (1988), 365-389.

[31] Kirillov A.N., Decomposition of symmetric and exterior powers of the adjoint representation of $\mathfrak{g l}(N)$. 1. Unimodality of principal specialization of the internal product of the Schur functions, Int. Jour. Mod. Phys. 7 (1992), p.545-579 
[32] Kirillov A.N., Unimodality of generalized Gaussian coefficients, C.R. Acad. Sci. Paris 315, Serie I (1992), p.497-501.

[33] Kirillov A.N., Dilogarithm identities, Progress of Theor. Phys. Suppl. 118 (1995), 61-142.

[34] Kirillov A.N., New combinatorial formula for modified Hall-Littlewood polynomials, Contemporary Mathematics 254 (2000), 283-333.

[35] Kirillov A.N., Combinatorics of Young tableaux and configurations (Russian), Transactions of SPb Math. Soc. 7 (2000), 100p.

[36] Kirillov A.N., Kostant partition functions for the root system of type A, Preprint, 1999, 20 p.

[37] Kirillov A.N., unpublished, 1987.

[38] Kirillov A.N. and Berenstein A.D., Groups generated by involutions, Gelfand-Tsetlin patterns, and combinatorics of Young tableaux, St. Petersburg Math. J. 7 (1996), no.1, $77-127$.

[39] Kirillov A.N. and Reshetikhin N.Yu., The Bethe ansatz and combinatorics of the Young tableaux (Russian), Zap. Nauch. Semin. LOMI, 155 (1986), p.65-115, translation in Journal of Soviet Math. 41 (1988), p.925-955.

[40] Kirillov A.N. and Shimozono M., A generalization of the Kostka-Foulkes polynomials, Preprint math.QA/9803062, 1998, 37p.

[41] Kirillov A.N., Schilling A. and Shimozono M., A bijection between Littlewood-Richardson tableaux and rigged configurations, Preprint math.QA/9901037, 1999, 66p.

[42] Kostant B., Lie group representations on polynomial rings, Amer. J. Math. 85 (1963), 327-404.

[43] Lascoux A., Leclerc B. and Tibon J.-Y., Ribbon tableaux, Hall-Littlewood functions, quantum affine algebras, and unipotent varieties, J. Math. Phys. 38 (1997), 1041-1068.

[44] Lascoux A. and Schutzenberger M.-P., Sur une conjecture de H.O. Foulkes, C.R. Acad. Sci. Paris 286A (1978), 323-324.

[45] Lascoux A. and Schutzenberger M.-P., La monoide plaxique, in Non-commutative structures in Algebra and Geometric Combinatorics, ROMA, CNR, 1981, 129-156.

[46] Littlewood D.E., On the Kronecker product of symmetric group representations, J. London Math. Soc. 31 (1956), 89-93.

[47] Littlewood D.E., On certain symmetric functions, Proc. London Math. Soc. 11 (1961), 485-498. 
[48] Lusztig G., Green polynomials and singularities of unipotent classes, Adv. in Math. 42 (1981), 169-178.

[49] Lusztig G., Singularities, character formulas, weight multiplicities, Asterisque 101-102 (1983), 208-229.

[50] Macdonald I.G., An elementary proof of a q-binomial identity, in D. Stanton (ed), qSeries and Partitions, IMA Volumes in Math. and its Appl. 18, Spring-Verlag, NY, 1989.

[51] Macdonald I.G., Notes on Schubert polynomials, Publications du LCIM 6 (1991), Université du Québec à Montréal.

[52] Macdonald I.G., Symmetric functions and Hall polynomials, 2nd ed., Oxford, 1995.

[53] Morris A.O., The characters of the group $G L(n, q)$, Math. Zeitschr. 81 (1963), 112-123.

[54] Morris A.O., A survey on Hall-Littlewood functions and their applications to the representation theory, Lect. Notes in Math. 579 (1977), 136-154.

[55] Nakayashiki A. and Yamada Y., On spinon character formulas, in Frontiers in Quantum Field Theory, ed. Itoyama et al., World Scientific, Singapore, 1996, 367-371.

[56] O'Hara K., Unimodality of Gaussian coefficients: a constructive proof, J. Comb. Theory A 53 (1990), 29-52.

[57] Proctor R.A., Odd symplectic groups, Invent. Math. 92 (1988), 307-332.

[58] Reeder M., Exterior powers of the adjoint representation, Can. Journ. Math. 49 (1997), 133-159.

[59] Sagan B., The symmetric group, Wadsworth and Brooks, Pacific Grove, California, 1991.

[60] Scharf T., Thibon J.-Y. and Wybourne B.G., Powers of the Vandermonde determinant and the quantum Hall effect, Journ. Phys. A: Math. Gen. 27 (1994), 4211-4219.

[61] Schilling A. and Warnaar S. Ole, Inhomogeneous lattice paths, generalized Kostka polynomials and $A_{n-1}$ supernomials, Comm. Math. Phys. 202 (1999), 359-401.

[62] Shimozono M. and Weyman J., Graded characters of modules supported in the closure of a nilpotent conjugacy class, Preprint math/9804036, 31p.

[63] Schützenberger M.-P., Proptiétés nouvelles des tableaux de Young, Séminaire DelangerPisot-Poitou, 19 ém année: 1977/78, Théorie des noumbres, Fasc.2, Exp. No.26, 14pp., Secrétariat Math., Paris, 1978.

[64] Stanley R., The stable behavior of some characters of $S L(n, \mathbf{C})$, Linear and Multilinear Algebra 16 (1984), 3-27. 
[65] Stanley R., Log-concave and unimodal sequences in algebra, combinatorics, and geometry, Annals of the New York Academy of Sciences 576 (1989), 500-535.

[66] Stanley R., Enumerative Combinatorics, vols. 1 and 2, Cambridge University Press, 1999.

[67] Stembridge J.R., Rational tableaux and the tensor algebra of $\mathfrak{g l}(n)$, J. Combin. Theory Ser. A 46 (1987), 79-120.

[68] Stembridge J.R., First layer formulas for characters of $S L(n, \mathbb{C})$, Trans. Amer. Math. Soc. 299 (1987), 319-350.

[69] Terada I., A generalization of the length-maj symmetry and the variety of $N$-stable flags, Manuscript dated by May 2, 1996, 27p.

[70] Weyman J., The equations of conjugacy classes of nilpotent matrices, Invent. Math. 98 (1989), 229-245.

[71] Zeilberger D., Kathy O'Hara's constructive proof of the unimodality of the Gaussian polynomials, Amer. Math. Monthly 96 (1989), 590-602.

[72] Zeilberger D., Proof of conjecture of Chan, Robbins and Yuen, Electron. Trans. Numer. Anal. 9 (1999), 147-148 (electronic).

[73] Zelevinsky A.V., A generalization of the Littlewood-Richardson rule and the RobinsonSchensted-Knuth correspondence, Journ. Algebra 69 (1981), 82-94. 